\title{
Calculation of the Regulator of a Pure Cubic Field
}

\author{
By H. C. Williams, G. Cormack and E. Seah
}

\begin{abstract}
A description is given of a modified version of Voronoi's algorithm for obtaining the regulator of a pure cubic field $2(\sqrt[3]{D})$. This new algorithm has the advantage of executing relatively rapidly for large values of $D$. It also eliminates a computational problem which occurs in almost all algorithms for finding units in algebraic number fields. This is the problem of performing calculations involving algebraic irrationals by using only approximations of these numbers.

The algorithm was implemented on a computer and run on all values of $D$ $\left(\leqslant 10^{5}\right)$ such that the class number of $2(\sqrt[3]{D})$ is not divisible by 3 . Several tables summarizing the results of this computation are also presented.
\end{abstract}

1. Introduction. Let $\delta$ be the real root of

$$
x^{3}-B x^{2}+C x-D=0
$$

an irreducible cubic equation with rational integer coefficients $B, C, D$ and negative discriminant $\Delta$. Let $2(\delta)$ be the cubic field formed by adjoining $\delta$ to the rationals, and let $2[\delta]$ be the ring of integers in $2(\delta)$. The regulator of $2(\delta)$ is $R=\log \epsilon_{0}$, where $\epsilon_{0}(>1)$ is the fundamental unit of $Q(\delta)$.

When $B=C=0$, we say that $2(\delta)$ is a pure cubic field. In Williams [8] an attempt was made to tabulate several pure cubic fields $2(\sqrt[3]{D})$ which have $D$ a prime $\equiv-1(\bmod 3)$ and class number 1 . In doing this it was necessary to evaluate the value of $R$ for each $Q(\sqrt[3]{D})$. This was done by using the algorithm of Voronoi [6] as described in Delone and Faddeev [3, pp. 282-290] and Beach, Williams and Zarnke [2].

Calculations had to be terminated when $D>35100$ for two reasons. The first reason was the immense amount of time needed (up to 10 minutes of C.P.U. time on an IBM 370-168 computer) to calculate an individual $R$ value; the second, and more important reason, was that the values of $D$ were getting too large for the precision available to the computer, even using double-precision arithmetic. All methods of evaluating $R$ known to the authors, with one exception, require that it be possible to determine when an algebraic irrational $\alpha \in Q(\delta)$ exceeds zero. The computer can only calculate an approximation $A$ to $\alpha$, and when $D$ is large it is not always true that if $A>0$, then $\alpha>0$.

Received January 15, 1979; revised September 12, 1979.

AMS (MOS) subject classifications (1970). Primary 12A30, 12A50; Secondary 12-04. 
The purpose of this paper is to describe a modification of Voronoi's technique for calculating $R$ in $Q(\sqrt[3]{D})$ which (1) executes rapidly and (2) minimizes the number of calculations which have to be done with irrational numbers. The method of [3, pp. $290 \mathrm{ff}$.] satisfies (2) but requires large precision when $D$ is large and executes rather slowly. We also present, in the last section, some results of running the programs which were written to implement our algorithm.

In Table 1 we summarize some of the notation used in this paper.

TABLE 1

Symbol

Description

$Z \quad$ The set of rational integers

$\delta \quad$ The real zero of an irreducible (over the rationals)

cubic polynomial with negative discriminant $\Delta$

$2(\delta)$

The cubic field formed by adjoining $\delta$ to the rationals

$2[\delta]$

The ring of integers in $2[\delta]$

$M, N$

$M=\left(m_{1}+m_{2} \delta+m_{3} \delta^{2}\right) / \sigma, N=\left(n_{1}+n_{2} \delta+n_{3} \delta^{2}\right) / \sigma$, where

$m_{1}, m_{2}, m_{3}, n_{1}, n_{2}, n_{3}, \sigma \in Z, \sigma>0$, and

g.c.d. $\left(m_{1}, m_{2}, m_{3}, n_{1}, n_{2}, n_{3}, \sigma\right)=1$

$0 \quad$ A lattice with basis $[1, M, N]$

$R \quad$ The real lattice derived from 0

$\theta_{g}$

Relative minimum of the second kind adjacent to 1

in a lattice which has 1 as a relative minimum

$R_{1}$

Real lattice which has as a basis an integral basis of $2[\delta]$

$\theta_{g}^{(r)}\left(\theta_{g}\right)$

Relative minimum of the second kind adjacent to 1 in $R_{r}(R)$

$\theta_{h}^{(r)}$

An element of $R_{r}$ such that $\left[1, \theta_{g}^{(r)}, \theta_{h}^{(r)}\right]$ is a basis of $R_{r}$

$R_{r+1}$

Real lattice with basis $\left[1,1 / \theta_{g}^{(r)}, \theta_{h}^{(r)} / \theta_{g}^{(r)}\right]$

$\Theta_{r}$

$\Theta_{r}=\prod_{i=1}^{r-1} \theta_{g}^{(i)}$

$Q_{r}$

$Q_{r}=N\left(\Theta_{r}\right)$, the norm of $\Theta_{r}$ 
Symbol

Description

$$
\begin{aligned}
M_{r}, N_{r}, e_{r} \quad & M_{r}=\left(m_{1}+m_{2} \delta+m_{3} \delta^{2}\right) / \sigma_{r}, N_{r}=\left(n_{1}+n_{2} \delta+n_{3} \delta^{2}\right) / \sigma_{r} \text { where } \\
& m_{1}, m_{2}, m_{3}, n_{1}, n_{2}, n_{3}, \sigma_{r} \in Z, \sigma_{r}>0, \\
& \text { g.c.d. }\left(m_{1}, m_{2}, m_{3}, n_{1}, n_{2}, n_{3}, \sigma_{r}\right)=1 \text { and }\left[1, M_{r}, N_{r}\right] \text { is a } \\
& \text { basis of } R_{r} . e_{r}=m_{2} n_{3}-n_{2} m_{3}
\end{aligned}
$$

$$
\begin{array}{ll}
m_{1}^{*}, m_{2}^{*}, m_{3}^{*}, & \left(m_{1}^{*}+m_{2}^{*} \delta+m_{3}^{*} \delta^{2}\right) / \sigma_{r}=1 / \theta_{g}^{(r-1)} \\
n_{1}^{*}, n_{2}^{*}, n_{3}^{*} & \left(n_{1}^{*}+n_{2}^{*} \delta+n_{3}^{*} \delta^{2}\right) / \sigma_{r}=\theta_{h}^{(r-1)} / \theta_{g}^{(r-1)} \\
& \text { here } m_{1}^{*}, m_{2}^{*}, m_{3}^{*}, n_{1}^{*}, n_{2}^{*}, n_{3}^{*} \in Z \text { and } \\
& \text { g.c.d. }\left(m_{1}^{*}, m_{2}^{*}, m_{3}^{*}, n_{1}^{*}, n_{2}^{*}, n_{3}^{*}, \sigma_{r}\right)=1 \\
& \\
\Omega, \Omega^{\prime}, \Omega^{\prime \prime} & \Omega \text { is an element of } R_{r}, \text { i.e. } \\
& \Omega=a M_{r}+b N_{r}+c(a, b, c \in Z) . \\
& \text { Also } \Omega=\left(q_{1}+q_{2} \delta+q_{3} \delta^{2}\right) / \sigma_{r}, \text { where } \\
& q_{1}, q_{2}, q_{3} \in Z . \Omega^{\prime} \text { and } \Omega^{\prime \prime} \text { are the } \\
& \text { conjugates of } \Omega .
\end{array}
$$

$E_{3} \quad$ Euclidean 3-space

$C_{\Omega} \quad C_{\Omega}$ is the normed body of $\Omega$; that is

$C_{\Omega}=\left\{(x, y, z)\left|(x, y, z) \in E_{3},\right| x|\leqslant| \Omega \mid, y^{2}+z^{2} \leqslant \Omega^{\prime} \Omega^{\prime \prime}\right\}$

$\omega \quad \omega$ is the puncture $\left(\xi_{\omega}, \eta_{\omega}\right)$ of $\Omega$.

Here

$$
\begin{array}{ll}
\left(\xi_{\omega}, \eta_{\omega}\right) \quad \xi_{\omega}=\left(2 \Omega-\Omega^{\prime}-\Omega^{\prime \prime}\right) / 2, \\
\eta_{\omega}=\left(\Omega^{\prime}-\Omega^{\prime \prime}\right) / 2 i \quad\left(i^{2}=-1\right), \\
\zeta_{\Omega}=\left(\Omega^{\prime}+\Omega^{\prime \prime}\right) / 2 \\
\text { when } \delta^{3}=D(D \in Z), \\
\xi_{\omega}=3 \delta\left(q_{2}+q_{3} \delta\right) / 2 \sigma_{r}, \eta_{\omega}=\sqrt{3} \delta\left(q_{2}-q_{3} \delta\right) / 2 \sigma_{r} \\
\zeta_{\Omega}=\left(2 q_{1}-q_{2} \delta-q_{3} \delta^{2}\right) / 2 \sigma_{r} .
\end{array}
$$

$$
q_{1}^{\prime}, q_{2}^{\prime}, q_{3}^{\prime} \quad q_{1}^{\prime}=q_{1}^{2}-D q_{2} q_{3}, q_{2}^{\prime}=D q_{3}^{2}-q_{1} q_{2}, q_{3}^{\prime}=q_{2}^{2}-q_{1} q_{3}
$$

$\mu, \nu \quad$ The punctures of $M_{r}$ and $N_{r}$ respectively

$\begin{array}{ll}g(\kappa) & g(\kappa)=\left(\sqrt{3}(\kappa+1 / 2)-\sqrt{1-(\kappa+1 / 2)^{2}}\right) / 2 \\ \beta & \beta=(2-\sqrt{3}) / 4\end{array}$


Symbol

Description

\begin{tabular}{ll}
$K_{1}(a, b)$ & $K_{1}(a, b)=\left(\begin{array}{ll}a & 1 \\
b & 0\end{array}\right), K_{2}(a, b)=\left(\begin{array}{ll}1 & a \\
0 & b\end{array}\right), K_{3}(a, b)=\left(\begin{array}{ll}0 & a \\
1 & b\end{array}\right)$ \\
$K_{3}(a, b)$ & \\
\hline$E_{r}(a, b)$ & $E_{r}=\left|\xi_{\mu} \eta_{\nu}-\xi_{\nu} \eta_{\mu}\right|$ \\
\hline$f(x, y)$ & $f(x, y)=x^{2}+|x y|+y^{2}$ \\
\hline$x_{\omega}, y_{\omega}$ & $x_{\omega}=I_{1} q_{2}+q_{3}\left[I_{1} \delta\right], y_{\omega}=I_{1} q_{2}-q_{3}\left[I_{1} \delta\right]$ \\
\hline $\bar{y}_{\omega}$ & $\bar{y}_{\omega}=\left[I_{2} \sqrt{3} \delta\right] q_{2}-q_{3}\left[I_{2} \sqrt{3} \delta^{2}\right]$ \\
\hline$X_{\omega}, Y_{\omega}$ & $X_{\omega}=\left[I_{3} \delta\right] q_{2}+q_{3}\left[I_{3} \delta^{2}\right], Y_{\omega}=\left[I_{3} \delta\right] q_{2}-q_{3}\left[I_{3} \delta^{2}\right]$
\end{tabular}

2. Preliminary Observations and Definitions. For a more general discussion of the ideas presented below see [3] and Steiner [5].

Let $O$ be a lattice [3] with basis $[1, M, N]$, where

$$
M=\left(m_{1}+m_{2} \delta+m_{3} \delta^{2}\right) / \sigma, \quad N=\left(n_{1}+n_{2} \delta+n_{3} \delta^{2}\right) / \sigma,
$$

$\sigma, m_{1}, m_{2}, m_{3}, n_{1}, n_{2}, n_{3} \in Z$ (the set of rational integers), $\sigma>0$, g.c.d. $\left(\sigma, m_{1}, m_{2}, m_{3}, n_{1}, n_{2}, n_{3}\right)=1$, and $\delta$ is defined as in the first paragraph of

Section 1 . Then $O$ is made up of the collection of ordered triples $\left(\Omega, \Omega^{\prime}, \Omega^{\prime \prime}\right)$, where

$$
\Omega=x+y M+z N, \quad x, y, z \in Z,
$$

and $\Omega^{\prime}, \Omega^{\prime \prime}$ are the conjugate roots of $\Omega$. Since $\Omega^{\prime}$ and $\Omega^{\prime \prime}$ are complex, we often discuss the real lattice $R$ which is the collection of points $\left(\Omega,\left(\Omega^{\prime}-\Omega^{\prime \prime}\right) / 2 i\right.$, $\left(\Omega^{\prime}+\Omega^{\prime \prime}\right) / 2$ ) of $E_{3}$ (Euclidean 3-space). Here $i^{2}=-1$. Since, to each $\Omega$, there corresponds a unique point of $R$, we often identify this point of $R$ by using the symbol $\Omega$ only and writing

$$
\Omega \approx\left(\Omega,\left(\Omega^{\prime}-\Omega^{\prime \prime}\right) / 2 i,\left(\Omega^{\prime}+\Omega^{\prime \prime}\right) / 2\right) .
$$

We define the value of $e$ for $R$ (or 0 ) as

$$
e=m_{2} n_{3}-m_{3} n_{2} \text {. }
$$

If $\Omega \in R$, we define its parameters to be $|\Omega|$ and

$$
\Omega^{\prime} \Omega^{\prime \prime}=\left(\left(\Omega^{\prime}-\Omega^{\prime \prime}\right) / 2 i\right)^{2}+\left(\left(\Omega^{\prime}+\Omega^{\prime \prime}\right) / 2\right)^{2} .
$$

We also define the norm of $\Omega$, written $N(\Omega)$, to be $\Omega \Omega^{\prime} \Omega^{\prime \prime}$ and the trace of $\Omega$ to be 
$\operatorname{Tr}(\Omega)=\Omega+\Omega^{\prime}+\Omega^{\prime \prime}$. Note that since $\Omega^{\prime} \Omega^{\prime \prime}=\left|\Omega^{\prime}\right|^{2} \geqslant 0$, then $\Omega$ and $N(\Omega)$ have the same sign. It should also be noted that [3, p. 274] if $\Omega$ and $\Phi$ are two points of $R$ such that $\Phi^{\prime} \Phi^{\prime \prime}=\Omega^{\prime} \Omega^{\prime \prime}$, then $\Phi= \pm \Omega$.

If $\Omega \in R$, we call the collection of all points $(x, y, z) \in E_{3}$ such that $|x| \leqslant|\Omega|$ and $y^{2}+z^{2} \leqslant \Omega^{\prime} \Omega^{\prime \prime}$ the normed body $C_{\Omega}$ of $\Omega$. That is, the normed body of $\Omega$ is a right circular cylinder of radius $\sqrt{\Omega^{\prime} \Omega^{\prime \prime}}$ and length $2|\Omega|$. It is oriented in $E_{3}$ as illustrated in Figure 1.

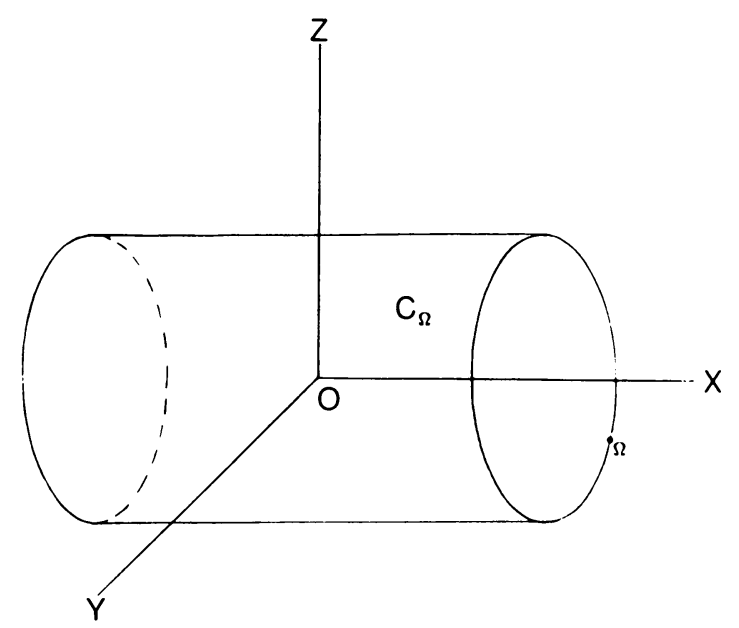

Figure 1

We say that $\Omega(\neq 0)$ is a relative minimum of $R$ (or 0 ) if the only points of $R$ which are in the normed body of $\Omega$ are $\Omega,-\Omega$ and 0 . For example, if $[1, M, N]$ is an integral basis of $2[\delta]$, then the point $1 \approx(1,0,1)$ is a relative minimum of $R$. For the norm of 1 is 1 and the value of $\Omega \Omega^{\prime} \Omega^{\prime \prime}$ for any $\Omega \in R$ is a rational integer; consequently, no point of $R$ except 0,1 and -1 can lie in the normed body of 1 . For the same reason we see that any unit $\epsilon$ of $2(\delta)$ must also be a relative minimum of $R$.

If $\Omega, \Phi$ are relative minima of $R$ such that

$$
0<\Phi<\Omega, \quad \Phi^{\prime} \Phi^{\prime \prime}>\Omega^{\prime} \Omega^{\prime \prime}
$$

and there does not exist a $\Psi \in R$ such that $\Phi<\Psi<\Omega$ and $\Psi^{\prime} \Psi^{\prime \prime}<\Phi^{\prime} \Phi^{\prime \prime}$, we call $\Phi$ the relative minimum of the first kind adjacent to $\Omega$; and we call $\Omega$ the relative minimum of the second kind adjacent to $\Phi$. See Figure 2 below.

Geometrically, we see that, given $\Phi$, we find $\Omega$ by increasing the length of the cylinder $C_{\Phi}$ until it includes a nonzero point of $R$. The first such point encountered is $\Omega$. That $\Omega$ must exist is guaranteed by the lemma of Minkowski [3, p. 80] . For a given $\Omega$, we find $\Phi$ by increasing the radius of the cylinder $C_{\Omega}$ until it includes a point $\Phi$ of $R$ such that $\Phi>0$.

Consider now the sequence

$$
\Theta_{1}, \Theta_{2}, \Theta_{3}, \ldots, \Theta_{n}, \ldots,
$$

where $\Theta_{1}$ is a relative minimum of $R$ and $\Theta_{i+1}$ is the relative minimum of the first kind adjacent to $\Theta_{i}$ for $i=1,2,3, \ldots$. We call such a sequence a chain of relative minima of the first kind. 


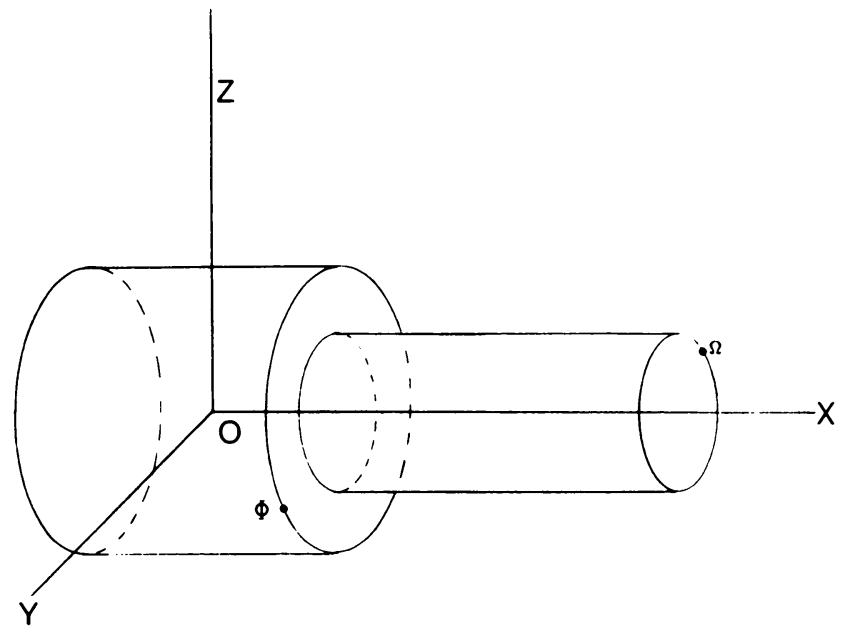

FIgURE 2

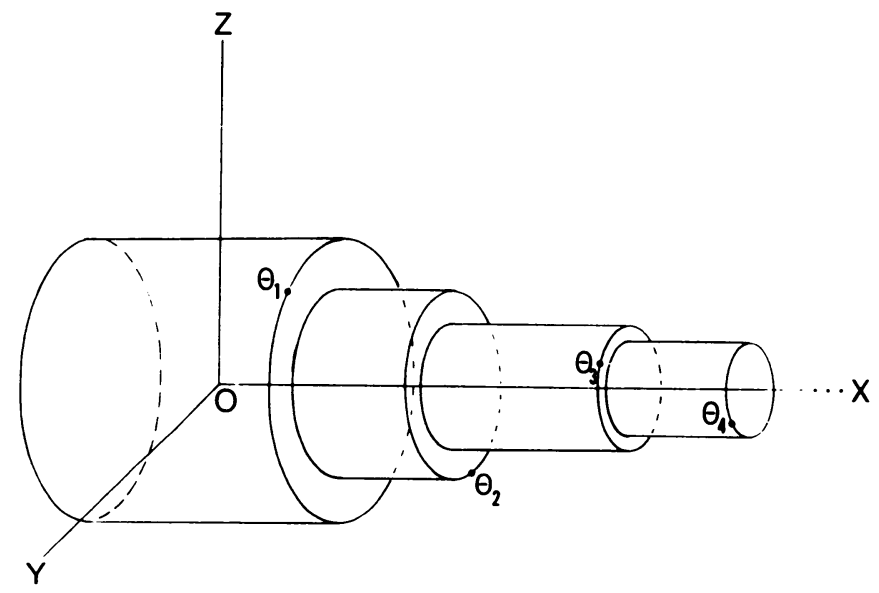

Figure 3

If $\Theta_{i+1}$ is the relative minimum of the second kind adjacent to $\Theta_{i}$, we call (2.1) a chain of relative minima of the second kind (see Figure 3). Voronoi's algorithm for determining $\epsilon_{0}^{-1}\left(\epsilon_{0}\right)$ consists of a method for obtaining a chain of relative minima of the first (second) kind for $R$ when $R$ has as a basis an integral basis of $2[\delta]$ and $\Theta_{1}$ $=1$, together with a method for determining which member of the chain is $\epsilon_{0}^{-1}\left(\epsilon_{0}\right)$.

Suppose the basis of $R=R_{1}$ is $[1, M, N]$, where $[1, M, N]$ is an integral basis of $2[\delta]$; and suppose we have found the relative minimum of the second kind $\Theta_{2}$ adjacent to $\Theta_{1}=1$. Put $\theta_{g}^{(1)}=\Theta_{2}$ and suppose $\left[1, \theta_{g}^{(1)}, \theta_{h}^{(1)}\right]$ is a basis of $R_{1}$. We let $R_{2}$ be the real lattice with basis $\left[1,1 / \theta_{g}^{(1)}, \theta_{h}^{(1)} / \theta_{g}^{(1)}\right]$. Clearly 1 is a relative minimum of $R_{2}$, and we find $\theta_{g}^{(2)}$, the relative minimum of the second kind adjacent to 1 in $R_{2}$ and $\Theta_{3}=\theta_{g}^{(1)} \theta_{g}^{(2)}$. We continue in this way finding $\left[1, \theta_{g}^{(n)}, \theta_{h}^{(n)}\right]$ a basis of $R_{n}$ and then defining $R_{n+1}$ as having a basis $\left[1,1 / \theta_{g}^{(n)}, \theta_{h}^{(n)} / \theta_{g}^{(n)}\right]$ and determining $\theta_{g}^{(n+1)}$, the relative minimum of the second kind adjacent to 1 in $R_{n+1}$. We 
have $\Theta_{n+2}=\theta_{g}^{(1)} \theta_{g}^{(2)} \cdots \theta_{g}^{(n+1)}$. When for some $k(>1), R_{k}$ and $R_{1}$ are the same lattice or, equivalently, $N\left(\Theta_{k}\right)=1$ (and $k$ is the least integer $(>1$ ) such that this is so), then

$$
\epsilon_{0}=\Theta_{k}=\theta_{g}^{(1)} \theta_{g}^{(2)} \cdots \theta_{g}^{(k-1)}
$$

and

$$
R=\log \epsilon_{0}=\sum_{i=1}^{k-1} \log \theta_{g}^{(i)} .
$$

In a later section we shall require the following theorem concerning $\theta_{g}^{(r)}$.

THEOREM 2.1. If $\theta_{g}^{(r)}$ is the relative minimum of the second kind adjacent to 1 in $R_{r}$, then $1 / \theta_{g}^{(r)}$ is the relative minimum of the first kind adjacent to 1 in $R_{r+1}$.

Proof. Let $\left[1, \theta_{g}^{(r)}, \theta_{h}^{(r)}\right]$ be a basis of $R_{r}$; then $\left[1,1 / \theta_{g}^{(r)}, \theta_{h}^{(r)} / \theta_{g}^{(r)}\right]$ is a basis of $R_{r+1}$. Also, since $\theta_{g}^{(r)}$ is the relative minimum of the second kind adjacent to 1 in $R_{r+1}$, we have $1 / \theta_{g}^{(r)}<1$ and $1 / \theta_{g}^{(r)^{\prime}} \theta_{g}^{(r) "}>1$.

Let $\Psi$ be the relative minimum of the first kind adjacent to 1 in $R_{r+1}$. Now if $\Theta \in R_{r+1}, \Theta \neq \Psi$ and $0<\Theta<1$, we must have $\Theta^{\prime} \Theta^{\prime \prime}>\Psi^{\prime} \Psi^{\prime \prime}$; for, if $0<\Theta<\Psi$, then since $C_{\Psi}$ contains no points of $R_{r+1}$ except for $0, \Psi$ and $-\Psi$, we get $\Theta^{\prime} \Theta^{\prime \prime}>$ $\Psi^{\prime} \Psi^{\prime \prime}$. On the other hand, if $\Psi<\Theta<1$, then $\Theta^{\prime} \Theta^{\prime \prime}>\Psi^{\prime} \Psi^{\prime \prime}$ by definition of the relative minimum of the first kind adjacent to 1 . Thus, if $\Theta=1 / \theta_{g}^{(r)}$, we see that

$$
0<\Psi<1 \text { and } \Psi^{\prime} \Psi^{\prime \prime}<1 / \theta_{g}^{(r)}{ }^{\prime} \theta_{g}^{(r) "} .
$$

Since $\Psi=a+b / \theta_{g}^{(r)}+c \theta_{h}^{(r)} / \theta_{g}^{(r)}(a, b, c \in Z)$, we have

$$
\Omega=\theta_{g}^{(r)} \Psi=a \theta_{g}^{(r)}+b+c \theta_{h}^{(r)} \in R_{r}, \quad 0<\Omega<\theta_{g}^{(r)},
$$

and $\Omega^{\prime} \Omega^{\prime \prime}<1$. This contradicts the fact that $\theta_{g}^{(r)}$ is the relative minimum of the second kind adjacent to 1 in $R_{r}$.

We conclude this section with some simple results concerning the points of $R_{r}$ when $\delta^{3}=D(D \in Z)$. Note that when we deal with the case $\delta^{3}=D$, we assume that $D$ is not a perfect integral cube.

If $\Omega \in R_{r}$ and $\delta^{3}=D$, then

$$
\Omega=a M_{r}+b N_{r}+c,
$$

where $a, b, c \in Z,\left[1, M_{r}, N_{r}\right]$ is a basis of $R_{r}$, and

$$
M_{r}=\left(m_{1}+m_{2} \delta+m_{3} \delta^{2}\right) / \sigma_{r}, \quad N_{r}=\left(n_{1}+n_{2} \delta+n_{3} \delta^{2}\right) / \sigma_{r} .
$$

Hence $\Omega=\left(q_{1}+q_{2} \delta+q_{3} \delta^{2}\right) / \sigma_{r}$, where $q_{1}, q_{2}, q_{3} \in Z$. If $\Omega \approx(u, v, w)$ in $R_{r}$, then

$$
u=\Omega, \quad v=\frac{\sqrt{3}}{2 \sigma_{r}}\left(q_{2} \delta-q_{3} \delta^{2}\right), \quad w=\left(2 q_{1}-q_{2} \delta-q_{3} \delta^{2}\right) / 2 \sigma_{r}
$$

Also,

$$
\operatorname{Tr}(\Omega)=3 q_{1} / \sigma_{r}, \quad N(\Omega)=\left(q_{1}^{3}+D q_{2}^{3}+D^{2} q_{3}^{3}-3 D q_{1} q_{2} q_{3}\right) / \sigma_{r}^{3}
$$


and

$$
\Omega^{\prime} \Omega^{\prime \prime}=\left(q_{1}^{\prime}+q_{2}^{\prime} \delta+q_{3}^{\prime} \delta^{2}\right) / \sigma_{r}^{2}
$$

where

$$
q_{1}^{\prime}=q_{1}^{2}-D q_{2} q_{3}, \quad q_{2}^{\prime}=D q_{3}^{2}-q_{1} q_{2}, \quad q_{3}^{\prime}=q_{2}^{2}-q_{1} q_{3} .
$$

We also have $e_{r}=m_{2} n_{3}-m_{3} n_{2}$. Now an integral basis of $2[\delta]$ is given by $\left[1, \delta, \delta^{2} / g_{2}\right]$ when $D \neq \equiv \pm 1(\bmod 9)$ and by $\left[1, \delta,\left(\delta^{2} \pm g_{2}^{2} \delta+g_{2}^{2}\right) / 3 g_{2}\right]$ when $D \equiv$ $\pm 1(\bmod 9)$. Here we assume $D=g_{1} g_{2}^{2}$, where $g_{1}$ and $g_{2}$ are square free and $\left(g_{1}, g_{2}\right)=1$. Thus, $\sigma_{1}=e_{1}$.

3. Some Results Concerning the Bases of $R_{r}$. If $\left[1, M_{r}, N_{r}\right]$ is any basis of $R_{r}$, then

$$
\left(\begin{array}{c}
1 \\
M_{r} \\
N_{r}
\end{array}\right)=\frac{1}{\theta_{g}^{(r-1)}} J_{r-1}\left(\begin{array}{c}
1 \\
M_{r-1} \\
N_{r-1}
\end{array}\right),
$$

where $\left[1, M_{r-1}, N_{r-1}\right]$ is any basis of $R_{r-1}$ and $J_{r-1}$ is a $3 \times 3$ matrix with integer coefficients and $\left|J_{r-1}\right|= \pm 1$. From this we get the result

$$
\left(\begin{array}{c}
1 \\
M_{r} \\
N_{r}
\end{array}\right)=\frac{1}{\Theta_{r}} J\left(\begin{array}{c}
1 \\
M_{1} \\
N_{1}
\end{array}\right),
$$

where $J$ is a $3 \times 3$ matrix with integer coefficients such that $|J|= \pm 1$. It follows that $\Theta_{r}, \Theta_{r} M_{r}, \Theta_{r} N_{r} \in R_{1}$. In this section we restrict ourselves to the case in which the basis of $R_{1}$ is an integral basis for $Q[\delta]$, when $Q(\delta)$ is a pure cubic field, i.e. $\delta^{3}$ $=D$. Put $\sigma=\sigma_{1}$ and $Q_{r}=N\left(\Theta_{r}\right)$. The proof of the following theorem makes use of the methods of Section 36 of Voronoi [6].

THEOREM 3.1. If $\delta^{3}=D$ and $Q_{r}=N\left(\Theta_{r}\right)$, then $e_{r} \mid \sigma_{r}$ and

$$
Q_{r}=\sigma_{r}^{2} /\left|e_{r}\right| \sigma \text {. }
$$

Proof. Since $\Theta_{r}, \Theta_{r} M_{r}, \Theta_{r} N_{r} \in R_{1}$, we have

$$
\begin{array}{ll}
\sigma \Theta_{r}=t_{11}+t_{12} \delta+t_{13} \delta^{2}, & \sigma \Theta_{r} M_{r}=t_{21}+t_{22} \delta+t_{23} \delta^{2} \\
\sigma \Theta_{r} N_{r}=t_{31}+t_{32} \delta+t_{33} \delta^{2} & \left(t_{i j} \in Z\right) .
\end{array}
$$

If

$$
S=\left(\begin{array}{ccc}
\sigma & 0 & 0 \\
\bar{m}_{1} & \bar{m}_{2} & \bar{m}_{3} \\
\bar{n}_{1} & \bar{n}_{2} & \bar{n}_{3}
\end{array}\right),
$$

where $\sigma M_{1}=\bar{m}_{1}+\bar{m}_{2} \delta+\bar{m}_{3} \delta^{2}, \sigma N_{1}=\bar{n}_{1}+\bar{n}_{2} \delta+\bar{n}_{3} \delta^{2}$, then $T=J S$, where $T$ $=\left(t_{i j}\right)_{3 \times 3}$. It follows that $|T|= \pm e \sigma\left(e=e_{1}\right)$. 
Let $\Gamma=\Theta_{r}^{\prime} \Theta_{r}^{\prime \prime}$. Since $\Theta_{r} \in 2[\delta]$, we see that $\Gamma \in 2[\delta]$ and, therefore, $\sigma \Gamma=$ $g_{1}+g_{2} \delta+g_{3} \delta^{2}\left(g_{1}, g_{2}, g_{3} \in Z\right)$. Thus, we find that

$$
\begin{aligned}
\sigma^{2} Q_{r} & =\left(g_{1}+g_{2} \delta+g_{3} \delta^{2}\right)\left(t_{11}+t_{12} \delta+t_{13} \delta^{2}\right), \\
\sigma^{2} Q_{r} M_{r} & =\left(g_{1}+g_{2} \delta+g_{3} \delta^{2}\right)\left(t_{21}+t_{22} \delta+t_{23} \delta^{2}\right)=u_{1}+u_{2} \delta+u_{3} \delta^{2}, \\
\sigma^{2} Q_{r} N_{r} & =\left(g_{1}+g_{2} \delta+g_{3} \delta^{2}\right)\left(t_{31}+t_{32} \delta+t_{33} \delta^{2}\right)=v_{1}+v_{2} \delta+v_{3} \delta^{2},
\end{aligned}
$$

and

$$
\left(\begin{array}{ccc}
\sigma^{2} Q_{r} & 0 & 0 \\
u_{1} & u_{2} & u_{3} \\
v_{1} & v_{2} & v_{3}
\end{array}\right)=T\left(\begin{array}{ccc}
g_{1} & g_{2} & g_{3} \\
D g_{3} & g_{1} & g_{2} \\
D g_{2} & D g_{3} & g_{1}
\end{array}\right)
$$

Thus, by taking determinants of both sides, we find that

$$
\sigma^{2} Q_{r}\left(u_{2} v_{3}-v_{2} u_{3}\right)= \pm e \sigma^{4} N(\Gamma)= \pm e \sigma^{4} Q_{r}^{2} \quad \text { and } \quad u_{2} v_{3}-v_{2} u_{3}= \pm e \sigma^{2} Q_{r} .
$$

If we put $d^{*}=$ g.c.d. $\left(u_{1}, u_{2}, u_{3}, v_{1}, v_{2}, v_{3}, Q_{r} \sigma^{2}\right)$, we get $\sigma_{r}=Q_{r} \sigma^{2} / d^{*}$ and $e_{r}=$ $m_{2} n_{3}-n_{2} m_{3}= \pm e \sigma^{2} Q_{r} /\left(d^{*}\right)^{2}= \pm e \sigma_{r} / d^{*}$.

Since $\Gamma$ and $\Theta_{r} M_{r}, \Theta_{r} N_{r} \in 2[\delta]$, we have

$$
\Theta_{r} \Gamma M_{r}=\left(u_{1}+u_{2} \delta+u_{3} \delta^{2}\right) / \sigma^{2} \in Q[\delta]
$$

and

$$
\Theta_{r} \Gamma N_{r}=\left(v_{1}+v_{2} \delta+v_{3} \delta^{2}\right) / \sigma^{2} \in Q[\delta]
$$

therefore, $\sigma$ is a factor of g.c.d. $\left(u_{1}, u_{2}, u_{3}, v_{1}, v_{2}, v_{3}\right)$ and $\sigma \mid d^{*}$. Since $d^{*}=$ $\pm e \sigma_{r} / e_{r}$ and $\sigma=e$, we have the theorem.

Corollary 3.1.1. $\sigma \mid \sigma_{r}$.

COROLlaRY 3.1.2. $Q_{r}=1$ if and only if $\left|e_{r}\right|=\sigma_{r}=\sigma$.

THEOREM 3.2. If $\Omega \in R_{r}$ and $\Omega=\left(q_{1}+q_{2} \delta+q_{3} \delta^{2}\right) / \sigma_{r}$, then $\sigma\left|e_{r}\right| \sigma_{r}$ is a factor of $N\left(\sigma_{r} \Omega\right)$ and $e_{r} \mid q_{i}^{\prime}(i=1,2,3)$, where $q_{1}^{\prime}=q_{1}^{2}-D q_{2} q_{3}, q_{2}^{\prime}=D q_{3}^{2}-q_{1} q_{2}$, $q_{3}^{\prime}=q_{2}^{2}-q_{1} q_{3}$.

Proof. Since $\Omega \in R_{r}$, we have $\Omega=a M_{r}+b N_{r}+c(a, b, c \in Z)$; also, since $\Theta_{r}$, $\Theta_{r} M_{r}$ and $\Theta_{r} N_{r} \in Q[\delta]$ so is $\Lambda=\Theta_{r} \Omega \in Q[\delta]$. It follows from (3.1) that

$$
N\left(\sigma_{r} \Omega\right)=\sigma_{r}^{3} N(\Omega)=\sigma_{r}^{3} N(\Lambda) / Q_{r}=\sigma\left|e_{r}\right| \sigma_{r} N(\Lambda) .
$$

Since $N(\Lambda)$ is an integer, we have the first part of the theorem.

Now $\Omega^{\prime} \Omega^{\prime \prime} / N(\Omega)=1 / \Omega=\Theta_{r} / \Lambda=\Lambda^{\prime} \Lambda^{\prime \prime} \Theta_{r} / N(\Lambda)$ and consequently $\Omega^{\prime} \Omega^{\prime \prime}=$ $\Lambda^{\prime} \Lambda^{\prime \prime} \Theta_{r} / N\left(\Theta_{r}\right)$. Using the fact that $\sigma_{r}^{2} \Omega^{\prime} \Omega^{\prime \prime}=q_{1}^{\prime}+q_{2}^{\prime} \delta+q_{3}^{\prime} \delta^{2}$, we have

$$
q_{1}^{\prime}+q_{2}^{\prime} \delta+q_{3}^{\prime} \delta^{2}=\sigma\left|e_{r}\right| \Lambda^{\prime} \Lambda^{\prime \prime} \Theta_{r} \text {. }
$$

Since $\Theta, \Lambda^{\prime} \Lambda^{\prime \prime} \in 2[\delta]$, we have $\Lambda^{\prime} \Lambda^{\prime \prime} \Theta \in Q 2[\delta]$; and, therefore, $e_{r} \mid q_{i}^{\prime}(i=1,2,3)$.

In the remainder of this section we discuss a method of finding the basis [1, $\left.1 / \theta_{g}^{(r-1)}, \theta_{h}^{(r-1)} / \theta_{g}^{(r-1)}\right]$ of $R_{r}$ when we have the basis $\left[1, \theta_{g}^{(r-1)}, \theta_{h}^{(r-1)}\right]$ of $R_{r-1}$. 
Let $m_{1}^{\prime}=m_{1}^{2}-D m_{2} m_{3}, m_{2}^{\prime}=D m_{3}^{2}-m_{2} m_{1}, m_{3}^{\prime}=m_{2}^{2}-m_{1} m_{3}$, where $\theta_{g}^{(r-1)}$ $=\left(m_{1}+m_{2} \delta+m_{3} \delta^{2}\right) / \sigma_{r-1}$; we have

$$
\sigma_{r-1}^{2} \theta_{g}^{(r-1)^{\prime}} \theta_{g}^{(r-1)^{\prime \prime}}=m_{1}^{\prime}+m_{2}^{\prime} \delta+m_{3}^{\prime} \delta^{2}
$$

and

$$
\sigma_{r-1}^{3} N\left(\theta_{g}^{(r-1)}\right)=m_{1} m_{1}^{\prime}+D\left(m_{2} m_{3}^{\prime}+m_{3} m_{2}^{\prime}\right)
$$

Put $d_{1}=$ g.c.d. $\left(m_{1}^{\prime}, m_{2}^{\prime}, m_{3}^{\prime}\right), \bar{m}_{i}=m_{i}^{\prime} / d_{1}(i=1,2,3)$ and $\bar{\sigma}_{r}=m_{1} \bar{m}_{1}+$ $D\left(m_{2} \bar{m}_{3}+m_{3} \bar{m}_{2}\right)$. We have

$$
1 / \theta_{g}^{(r-1)}=\sigma_{r-1}\left(\bar{m}_{1}+\bar{m}_{2} \delta+\bar{m}_{3} \delta^{2}\right) / \bar{\sigma}_{r}
$$

and

$$
\theta_{h}^{(r-1)} / \theta_{g}^{(r-1)}=\left(n_{1}^{\prime}+n_{2}^{\prime} \delta+n_{3}^{\prime} \delta^{2}\right) / \bar{\sigma}_{r},
$$

where

$$
\begin{gathered}
n_{1}^{\prime}=\bar{m}_{1} n_{1}+D\left(\bar{m}_{2} n_{3}+\bar{m}_{3} n_{2}\right), \quad n_{2}^{\prime}=\bar{m}_{2} n_{1}+\bar{m}_{1} n_{2}+D \bar{m}_{3} n_{3}, \\
n_{3}^{\prime}=\bar{m}_{3} n_{1}+\bar{m}_{2} n_{2}+\bar{m}_{1} n_{3} .
\end{gathered}
$$

If $d_{2}=\left(n_{1}^{\prime}, n_{2}^{\prime}, n_{3}^{\prime}\right)$, we get

$$
n_{2}\left(\bar{m}_{2}^{2}-\bar{m}_{3} \bar{m}_{1}\right) \equiv n_{3}\left(D \bar{m}_{3}-\bar{m}_{1} \bar{m}_{2}\right) \quad\left(\bmod d_{2}\right)
$$

from the last two equations. Now

$$
\bar{m}_{2}^{2}-\bar{m}_{3} \bar{m}_{1}=m_{3} \bar{\sigma}_{r} / d_{1}, \quad D \bar{m}_{3}^{2}-\bar{m}_{1} \bar{m}_{2}=m_{2} \bar{\sigma}_{r} / d_{1} ;
$$

hence, $d_{2} d_{1} \mid \bar{\sigma}_{r} e_{r-1}$. Also, since $\Theta_{r}=\Theta_{r-1} \theta_{g}^{(r-1)}$, we have $Q_{r-1} N\left(\theta_{g}^{(r-1)}\right)=Q_{r}$.

From (3.1), we have $Q_{r-1}=\sigma_{r-1}^{2} / / e_{r-1} \mid \sigma$; thus, since $N\left(\theta_{g}^{(r-1)}\right)=d_{1} \bar{\sigma}_{r} / \sigma_{r-1}^{3}$, we get $d_{1} \bar{\sigma}_{r}=\sigma\left|e_{r-1}\right| \sigma_{r-1} Q_{r}$. If $d=\left(d_{2}, \sigma_{r-1}\right)=\left(n_{1}^{\prime}, n_{2}^{\prime}, n_{3}^{\prime}, \sigma_{r-1}\right)$, then $e_{r-1} d \mid d_{1} \bar{\sigma}_{r}$ and $d d_{1} \mid \bar{\sigma}_{r} e_{r-1} ;$ therefore, $d \mid \bar{\sigma}_{r}$. We have proved

THEOREM 3.3. If $\left[1, \theta_{g}^{(r-1)}, \theta_{h}^{(r-1)}\right]$ is a basis of $R_{r-1}$, then $\left[1, M_{r}, N_{r}\right]$ is a basis of $R_{r}$, where

$$
\begin{aligned}
& M_{r}=1 / \theta_{g}^{(r-1)}=\left(m_{1}^{*}+m_{2}^{*} \delta+m_{3}^{*} \delta^{2}\right) / \sigma_{r}, \\
& N_{r}=\theta_{h}^{(r-1)} / \theta_{g}^{(r-1)}=\left(n_{1}^{*}+n_{2}^{*} \delta+n_{3}^{*} \delta^{2}\right) / \sigma_{r},
\end{aligned}
$$

$\sigma_{r}=\bar{\sigma}_{r} / d, m_{i}^{*}=\sigma_{r-1} m_{i}^{\prime} / d d_{1}, n_{i}^{*}=n_{i}^{\prime} / d(i=1,2,3)$.

It should be noted here that since $d_{1}=\left|e_{r-1}\right| \sigma_{r} \sigma_{r-1}\left|e_{r}\right| d$, we have

$$
m_{i}^{\prime}=\left|e_{r-1}\right| \sigma_{r} m_{i}^{*}|| e_{r} \mid \quad(i=1,2,3) .
$$

Let $B=[1, M, N]$ be any basis of the lattice $R$, and let $K=\left(k_{i j}\right)_{2 \times 2}$ be a matrix with integer entries such that $|K|= \pm 1$. We say that we transform the basis $B$ by $K$ when we replace $[1, M, N]$ by $[1, \bar{M}, \bar{N}]$, where

$$
\bar{M}=\left(\bar{m}_{1}+\bar{m}_{2} \delta+\bar{m}_{3} \delta^{2}\right) / \sigma_{r}, \quad \bar{N}=\left(\bar{n}_{1}+\bar{n}_{2}+\bar{n}_{3} \delta^{2}\right) / \sigma_{r}
$$


and

$$
\left(\begin{array}{ll}
\bar{m}_{1} & \bar{n}_{1} \\
\bar{m}_{2} & \bar{n}_{2} \\
\bar{m}_{3} & \bar{n}_{3}
\end{array}\right)=\left(\begin{array}{ll}
m_{1} & n_{1} \\
m_{2} & n_{2} \\
m_{3} & n_{3}
\end{array}\right) K .
$$

Since $|K|= \pm 1$, we see that the transformed basis is also a basis of $R$. Now, if $m_{3}$ $\neq 0$ and

$$
K=\left(\begin{array}{cc}
1 & -k \\
0 & 1
\end{array}\right),
$$

where* $k=\left[n_{3} / m_{3}\right]$, then $\bar{m}_{2}=m_{2}, \bar{m}_{3}=m_{3}, \bar{n}_{2}=n_{2}-k m_{2}$, and $\bar{n}_{3}=n_{3}-$ $k m_{3}$; thus, $\left|\bar{n}_{3} / \bar{m}_{3}\right|<1$. Also, since $e_{r}=\bar{m}_{2} \bar{n}_{3}-\bar{m}_{3} \bar{n}_{2}$, we get $\left|\bar{n}_{2}\right|<\left|e_{r}\right| /\left|m_{3}\right|+$ $\left|m_{2}\right|$. If $m_{3}=0$ and $k=\left[n_{2} / m_{2}\right]$, we have $\left|\bar{n}_{2} / \bar{m}_{2}\right|<1$. Thus, if $[1, M, N]$ is a basis of $R$, we know that there exists a basis $[1, \bar{M}, \bar{N}]$ of $R$ such that if $m_{3} \neq 0$, then

$$
\bar{m}_{2}=m_{2}, \quad \bar{m}_{3}=m_{3}, \quad\left|\bar{n}_{3}\right|<\left|\bar{m}_{3}\right|, \quad\left|\bar{n}_{2}\right|<\left|e_{r}\right| /\left|m_{3}\right|+\left|m_{2}\right|
$$

or, if $m_{3}=0$, then

$$
\bar{m}_{2}=m_{2}, \quad \bar{m}_{3}=0, \quad\left|\bar{n}_{2}\right|<\left|\bar{m}_{2}\right|
$$

4. Some Lemmas Concerning the Lattice $R$. As in Section 2 we let $R$ be any lattice with basis $[1, M, N]$. Suppose further that $R$ has 1 as a relative minimum. In Section 5 we show how to determine a small set of possible values for $\theta_{g}$, the relative minimum of the second kind adjacent to 1 in $R$; however, in order to do this we must first prove a number of lemmas concerning $R$.

We define the puncture of any $\Omega \in R$ to be a point $\omega=\left(\xi_{\omega}, \eta_{\omega}\right)$ in the $x-y$ plane of $E_{3}$, where

$$
\xi_{\omega}=\left(2 \Omega-\Omega^{\prime}-\Omega^{\prime \prime}\right) / 2, \cdot \eta_{\omega}=\frac{\Omega^{\prime}-\Omega^{\prime \prime}}{2 i} \quad\left(i^{2}=-1\right)
$$

That is, if $\Omega \approx(u, v, w)$, then $\omega=F(u, v, w)$, where $F(u, v, w)=(u-w, v)$. Thus, $\omega$ is simply the point at which a line passing through $\Omega$ and parallel to the line joining the origin of $E_{3}$ to $(1,0,1)$ meets the $x-y$ plane. We denote the set of all these punctures by $L$. We also denote by $\zeta_{\Omega}$ the value of $\left(\Omega^{\prime}+\Omega^{\prime \prime}\right) / 2$; hence, $\Omega=\xi_{\omega}+\zeta_{\Omega}$. When $\delta^{3}=D$, we see that if $\Omega=\left(q_{1}+q_{2} \delta+q_{3} \delta^{2}\right) / \sigma$, then

$$
\begin{gathered}
\xi_{\omega}=3 \delta\left(q_{2}+q_{3} \delta\right) / 2 \sigma, \quad \eta_{\omega}=\sqrt{3} \delta\left(q_{1}-q_{2} \delta\right) / 2 \sigma \\
\zeta_{\Omega}=\left(2 q_{1}-q_{2} \delta-q_{3} \delta^{2}\right) / 2 \sigma .
\end{gathered}
$$

We note here that, if

$$
W=\left(\begin{array}{ll}
\xi_{\mu} & \xi_{\nu} \\
\eta_{\mu} & \eta_{\nu}
\end{array}\right),
$$

where $\left(\xi_{\mu}, \eta_{\mu}\right)$ and $\left(\xi_{\nu}, \eta_{\nu}\right)$ are, respectively, the punctures of $M$ and $N$, then when we transform the basis by $K$, we replace $W$ by $W K$.

*We denote by $[\alpha]$ that rational integer such that $0<\alpha-[\alpha]<1$; we also denote by $\{\alpha\}$ the value of $\alpha-[\alpha]$. 
Since $F$ is a linear mapping, we see that $L$ is additive; thus, if $\Phi$ and $\Psi$ are any two points of $R$ and $\Omega=a \Phi+b \Psi+c(a, b, c \in Z)$, then the puncture $\omega=$ $\left(\xi_{\omega}, \eta_{\omega}\right)$ of $\Omega$ is given by $\xi_{\omega}=a \xi_{\phi}+b \xi_{\psi}$ and $\eta_{\omega}=a \eta_{\phi}+b \eta_{\psi}$, where $\phi=\left(\xi_{\phi}, \eta_{\phi}\right)$, $\psi=\left(\xi_{\psi}, \eta_{\psi}\right)$ are the punctures of $\Phi$ and $\Psi$, respectively. Also, $\zeta_{\Omega}=a \zeta_{\Phi}+b \zeta_{\Psi}+c$. It follows that $L$ is a two-dimensional lattice with basis the punctures of $M$ and $N$. Thus, if $\Omega_{1}=a_{1} M+b_{1} N+c_{1}, \Omega_{2}=a_{2} M+b_{2} N+c_{2}\left(a_{1}, a_{2}, b_{1}, b_{2}, c_{1}, c_{2} \in Z\right)$, then $\Omega_{1}$ and $\Omega_{2}$ have the same puncture if and only if $a_{1}=a_{2}$, and $b_{1}=b_{2}$. Let $\Omega=a M+b N+c(a, b, c \in Z)$ have $\omega$ as its puncture. We say that $\Omega$ belongs to $\omega$ if $-1<\zeta_{\Omega}<1$. That is, $\Omega$ belongs to $\omega$ if it is one of the two points on either side of the $x-y$ plane of $E_{3}$ which has $\omega$ as a puncture and is closest to the $x-y$ plane. In Figure 4 below both $\Omega_{1}$ and $\Omega_{2}$ belong to $\omega$.

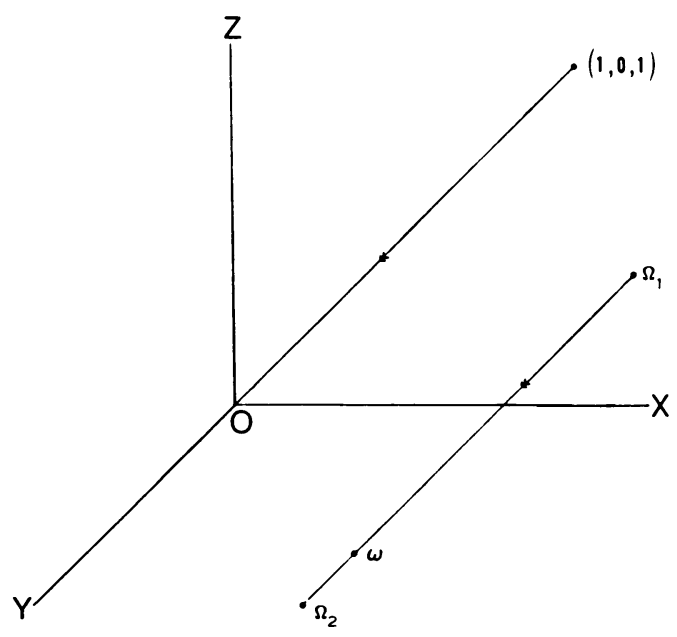

FIGURE 4

We have $\Omega_{1}-\Omega_{2}=1$ and $c_{1}=\left[-a \zeta_{M}-b \zeta_{N}\right]+1$.

Let $C$ be the set $\left\{(x, y, z) \mid(x, y, z) \in E_{3}\right.$ and $\left.z^{2}+y^{2} \leqslant 1\right\}$, i.e. the set of all points of $E_{3}$ in or on the cylinder $z^{2}+y^{2}=1$, and let $C_{1}$ be the normed body of 1 . Note that only two points $( \pm 1)$ of $R$ lie on the surface of $C$ and no points of $R$ except 0 and \pm 1 are contained in $C_{1}$. Note also that $\theta_{g} \in C$. We have the following

Definition of $\Omega^{*}$. Let $\Omega_{1}$ and $\Omega_{2}$ be the two points which belong to $\omega$. If just one of these is in $\mathcal{C}$, denote it by $\Omega^{*}$. If both are in $\mathcal{C}$, put $\Omega^{*}=\Omega_{1}$ if $\left|\Omega_{1}\right|<\left|\Omega_{2}\right|$; else put $\Omega^{*}=\Omega_{2}$. If neither $\Omega_{1}$ nor $\Omega_{2}$ is in $C, \Omega^{*}$ is not defined.

Note that in order for $\Omega \in \mathcal{C}$, we must have $\zeta_{\Omega}^{2}+\eta_{\omega}^{2}<1$; hence, if $\left|\eta_{\omega}\right|>1$, $\Omega^{*}$ does not exist. On the other hand, if $\left|\eta_{\omega}\right|<\sqrt{3} / 2$, then $\Omega^{*}$ must exist. Further, if $\left|\eta_{\omega}\right|>\sqrt{3} / 2$ and $\Omega^{*}$ exists, then $\left|\zeta_{\Omega *}\right|<1 / 2$ and $\Omega^{*}=c+a M+b N$, where $c=$ $\left[1 / 2-a \zeta_{M}-b \zeta_{N}\right]$. If $\left|\eta_{\omega}\right|<\sqrt{3} / 2$ and $\zeta_{\Omega_{2}}>-1 / 2$, then $\Omega_{2} \in \mathcal{C}$ and since $\Omega_{1}=$ $\Omega_{2}+1$, we must have $\Omega_{2}=\Omega^{*}$.

We are now able to present several lemmas. These results are analogous to results given in [3] for the case $\Delta>0$. Wada also made use of results of this type to produce his table [7]. 
Lemma 4.1. If $\omega=\left(\xi_{\omega}, \eta_{\omega}\right)$ is a puncture such that $\Omega^{*}(>0, \neq 1)$ exists, then

$$
-1 \leqslant \zeta_{\Omega} * 1-\sqrt{1-\eta_{\omega}^{2}}
$$

Proof. Since $\Omega^{*} \in C$ and $\Omega^{*}>0$, we must have $\Omega^{*}>1$; also, by definition $\left|\zeta_{\Omega^{*}}\right|<1$. If $\zeta_{\Omega^{*}} \geqslant 1-\sqrt{1-\eta_{\omega}^{2}}$, then $\left(\zeta_{\Omega^{*}}-1\right)^{2}+\eta_{\omega}^{2} \leqslant 1$ and, consequently, $\Omega^{*}-1 \in \mathcal{C}$. Since $0<\Omega^{*}-1<\Omega^{*}$, this contradicts the definition of $\Omega^{*}$.

LemmA 4.2. If $\omega=\left(\xi_{\omega}, \eta_{\omega}\right)$ is the puncture of $\Omega, \xi_{\omega}>0$, and $\left|\eta_{\omega}\right|<\sqrt{3} / 2$, then $\xi_{\omega}>\sqrt{1-\eta_{\omega}^{2}}$.

Proof. Since $\left|\eta_{\omega}\right|<\sqrt{3} / 2$, there must exist $\Omega^{*} \in R$ with puncture $\omega$. Since $\xi_{\omega}>0$ and no point of $R$ except 0 exists within $C_{1}$, we must have $\Omega^{*}>1$; thus, since $\Omega^{*}=\xi_{\omega}+\zeta_{\Omega^{*}}$ and $\zeta_{\Omega *}<1-\sqrt{1-\eta_{\omega}^{2}}$, we get $\xi_{\omega}>\sqrt{1-\eta_{\omega}^{2}}$.

Lemma 4.3. Let $\omega=\left(\xi_{\omega}, \eta_{\omega}\right)$ be the puncture of a point $\Omega$ such that $\xi_{\omega}>$ $0,\left|\eta_{\omega}\right|<\sqrt{3} / 2$ and let $\tau=\left(\xi_{\tau}, \eta_{\tau}\right)$ be the puncture of a point $T \in \mathcal{C}$. If $\xi_{\tau}>\xi_{\omega}$ and $\eta_{\tau} \eta_{\omega}>0$, then $T>\Omega^{*}$.

Proof. Suppose $T<\Omega^{*}$. Since $\xi_{\tau}>\xi_{\omega}$ and $T=\xi_{\tau}+\zeta_{T}<\xi_{\omega}+\zeta_{\Omega^{*}}=\Omega^{*}$, we see that $\zeta_{T}<\zeta_{\Omega^{*}}$. Since $\zeta_{T}>-1$ and $-\zeta_{\Omega^{*}}>-1 / 2$, we have $-3 / 2<\zeta_{T}-\zeta_{\Omega^{*}}$ $<0$. Thus, the absolute value of one of $\zeta_{T}-\zeta_{\Omega *}$ or $\zeta_{T}-\zeta_{\Omega *}+1$ must be less than 1/2. If $\left|\eta_{\tau}-\eta_{\omega}\right|<\sqrt{3} / 2$, one of $T-\Omega^{*}$ or $T-\Omega^{*}+1 \in \mathcal{C}$.

Since $\eta_{\tau}$ and $\eta_{\omega}$ have the same sign, $\left|\eta_{\tau}-\eta_{\omega}\right|=|| \eta_{\tau}|-| \eta_{\omega}||$. If $\left|\eta_{\tau}-\eta_{\omega}\right|$ $=\left|\eta_{\omega}\right|-\left|\eta_{\tau}\right|$, then $\left|\eta_{\tau}-\eta_{\omega}\right|<\left|\eta_{\omega}\right|<\sqrt{3} / 2$. Suppose that $\left|\eta_{\tau}-\eta_{\omega}\right|=\left|\eta_{\tau}\right|-$ $\left|\eta_{\omega}\right|>\sqrt{3} / 2$; then $\left|\eta_{\tau}\right|>\sqrt{3} / 2$. We now assume without loss of generality that $\eta_{\omega}$ $>0$ and consider Figure 5 below.

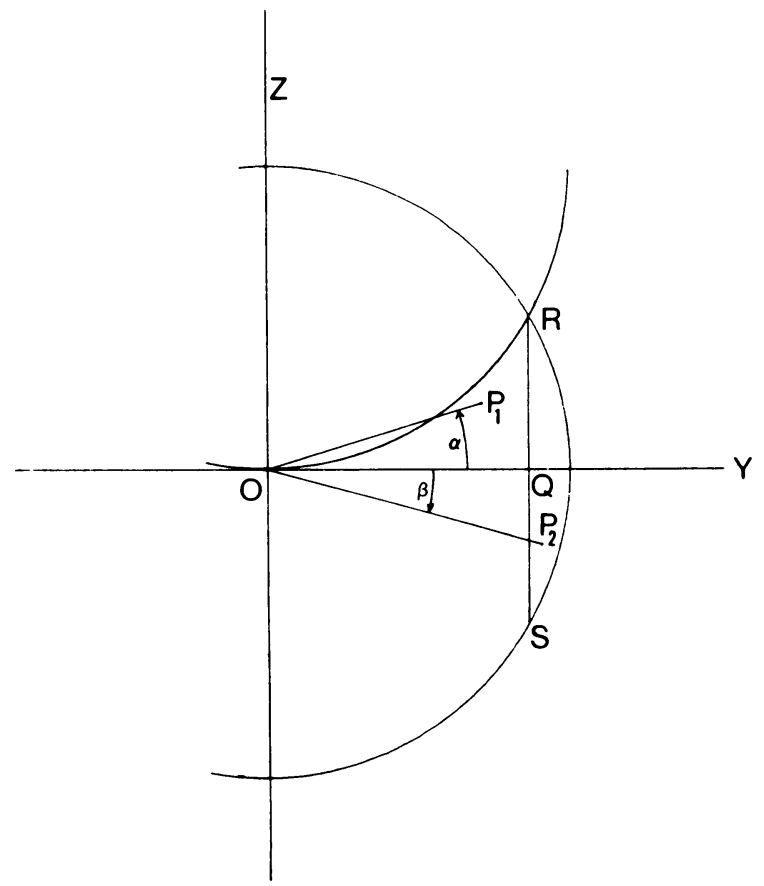

FIGURE 5 
$\overline{P_{1} P_{2}}$, then

Here $P_{1}=\left(\eta_{\omega}, \zeta_{\Omega}\right), P_{2}=\left(\eta_{\tau}, \zeta_{T}\right)$. If we let $d_{1}=\overline{O P}_{1}, d_{2}=\overline{O P}_{2}, d_{3}=$

$$
\left(\eta_{\tau}-\eta_{\omega}\right)^{2}+\left(\zeta_{T}-\zeta_{\Omega *}\right)^{2}=d_{3}^{2}=d_{1}^{2}+d_{2}^{2}-2 d_{1} d_{2} \cos (\alpha+\beta) .
$$

Now $\overline{O Q}=\sqrt{3} / 2$ and $\overline{Q R}=\overline{Q S}=1 / 2$; thus, if $P_{1}$ lies above the line $O P_{2}$ or if $P_{1}$ lies below $O P_{2}$ and $\zeta_{T}>0$, then $\alpha+\beta<\pi / 3$ and $\cos (\alpha+\beta)>1 / 2$. It follows that

$$
d_{3}^{2}<d_{1}^{2}+d_{2}^{2}-d_{1} d_{2} \leqslant \max \left(d_{1}^{2}, d_{2}^{2}\right) \quad\left(d_{1}, d_{2}>0\right) .
$$

Since $d_{1}$ and $d_{2}<1$, we have $d_{3}^{2}<1$. If $P_{1}$ lies below $O P_{2}$ and $\zeta_{T}<0$, then since $\zeta_{\Omega^{*}}>\zeta_{T}$, the angle between $O P_{1}$ and $P_{1} P_{2}$ exceeds $\pi / 2$; hence, $d_{3}<d_{2}<1$. In each of the cases above we find $d_{3}<1$ and, thus, $\left(\eta_{\tau}-\eta_{\omega}\right)^{2}+\left(\zeta_{T}-\zeta_{\Omega *}\right)^{2}<1$ or $T-\Omega^{*} \in \mathcal{C}$.

Thus, under the assumption that $T<\Omega^{*}$, we see that either $T-\Omega^{*}+1$ or $T-\Omega^{*} \in \mathrm{C}$. If, however, $T-\Omega^{*} \in \mathrm{C}$, then $\zeta_{T}-\zeta_{\Omega}>-1$ and, consequently, $T-\Omega^{*}$ $>\zeta_{T}-\zeta_{\Omega^{*}}>-1$. Since $T-\Omega^{*}<0$, we have $T-\Omega^{*} \in C_{1}$, which is impossible. If $T-\Omega^{*} \notin C_{1}$, then $-3 / 2<\zeta_{T}-\zeta_{\Omega^{*}}<-1 / 2$ or $T-\Omega^{*}+1>-1 / 2$; also, $T-\Omega^{*}$ $+1<1$; and we have $T-\Omega^{*}+1 \in C_{1}$, which is also impossible. It follows that $T$ $>\Omega *$.

LEMMA 4.4. Let $g(\kappa)=\left(\sqrt{3}(\kappa+1 / 2)-\sqrt{1-(\kappa+1 / 2)^{2}}\right) / 2$. If $0 \leqslant \kappa \leqslant$ $(\sqrt{3}-1) / 2$ and $g(\kappa) \leqslant \lambda \leqslant 1$, then

$$
(\kappa+1 / 2) \sqrt{1-\lambda^{2}}-\lambda \sqrt{1-(\kappa+1 / 2)^{2}} \leqslant 1 / 2 .
$$

Proof. If $\lambda \geqslant\left(\sqrt{3}(\kappa+1 / 2)-\sqrt{1-(\kappa+1 / 2)^{2}}\right) / 2$, then

$$
\lambda^{2}+\lambda \sqrt{1-(\kappa+1 / 2)^{2}}+1 / 4-(\kappa+1 / 2)^{2} \geq 0
$$

consequently,

$$
(\kappa+1 / 2)^{2}\left(1-\lambda^{2}\right) \leqslant \lambda^{2}\left(1-(\kappa+1 / 2)^{2}\right)+\lambda \sqrt{1-(\kappa+1 / 2)^{2}}+1 / 4,
$$

and

$$
(\kappa+1 / 2) \sqrt{1-\lambda^{2}} \leqslant \lambda \sqrt{1-(\kappa+1 / 2)^{2}}+1 / 2 .
$$

LEMmA 4.5. Let $\omega=\left(\xi_{\omega}, \eta_{\omega}\right)$ be a puncture of a point $\Omega$ such that $\left|\eta_{\omega}\right|<$ $1 / 2+\kappa(0 \leqslant \kappa<(\sqrt{3}-1) / 2)$, and let $\tau=\left(\xi_{\tau}, \eta_{\tau}\right)$ be the puncture of a point $T \in \mathcal{C}$. Suppose further that $\xi_{\tau}=1+\lambda+\xi_{\omega}$, where $\lambda \geqslant 0$. If $\lambda \geqslant g(\kappa)$, we must have $\Omega^{*}<T$.

Proof. Suppose $\Omega^{*}>T$; since $\xi_{\tau}=1+\lambda+\xi_{\omega}$, we have $1+\lambda-\zeta_{\Omega}+\zeta_{T}$ $<0$. From this we see that, since $\zeta_{T}>-1$, we get $\zeta_{\Omega^{*}}>\lambda \geqslant 0$. Also, $\zeta_{\Omega^{*}}<1 / 2$ (Lemma 4.1) and $\zeta_{T}-\zeta_{\Omega^{*}}>-3 / 2$; thus,

$$
-3 / 2<\zeta_{T}-\zeta_{\Omega *}<-\lambda-1 \text { and } \lambda<\zeta<1 / 2 \text {, }
$$

where $\zeta=\zeta_{\Omega^{*}}-\zeta_{T}-1$. Now $\left|\eta_{\omega}\right|<1 / 2+\kappa<\sqrt{3} / 2$ and (Lemma 4.1)

$$
0<\zeta_{\Omega} * 1-\sqrt{1-\eta_{\omega}^{2}}<1-\sqrt{1-(1 / 2+\kappa)^{2}}
$$


hence,

$$
\left|\zeta_{T}\right| \geqslant \zeta_{\Omega *}-\zeta_{T}-\zeta_{\Omega *}>\zeta+\sqrt{1-(1 / 2+\kappa)^{2}} .
$$

If $T-\Omega^{*}+1$ is not in $C$, then

$$
\left(\zeta_{T}-\zeta_{\Omega^{*}}+1\right)^{2}+\left(\eta_{\tau}-\eta_{\omega}\right)^{2}>1 \text { and }\left|\eta_{\tau}-\eta_{\omega}\right|>\sqrt{1-\zeta^{2}} \text {. }
$$

Hence,

$$
\left|\eta_{\tau}\right| \geqslant\left|\eta_{\tau}-\eta_{\omega}\right|-\left|\eta_{\omega}\right|>\sqrt{1-\zeta^{2}}-\kappa-1 / 2
$$

and

$$
\begin{aligned}
\zeta_{T}^{2}+\eta_{\tau}^{2} & >\left(\zeta+\sqrt{1-(1 / 2+\kappa)^{2}}\right)^{2}+\left(\sqrt{1-\zeta^{2}}-\kappa-1 / 2\right)^{2} \\
& =2-2(\kappa+1 / 2) \sqrt{1-\zeta^{2}}+2 \zeta \sqrt{1-(\kappa+1 / 2)^{2}} \\
& >2-2(\kappa+1 / 2) \sqrt{1-\lambda^{2}}+2 \lambda \sqrt{1-(\kappa+1 / 2)^{2}} \\
& \geqslant 1 \quad \text { (by Lemma 4.4). }
\end{aligned}
$$

Since $T \in \mathcal{C}$, it follows that we must have $T-\Omega^{*}+1 \in \mathcal{C}$.

Since $\Omega^{*}>T$, we have $T-\Omega^{*}+1<1$ and since

$$
T-\Omega^{*}+1=\xi_{\tau}+\zeta_{T}-\xi_{\omega}-\zeta_{\Omega^{*}}+1=2+\zeta_{T}-\zeta_{\Omega *}+\lambda,
$$

we have $T-\Omega^{*}+1>1 / 2$. Hence, $T-\Omega^{*}+1 \in C_{1}$, i.e. $T-\Omega^{*}+1=0$; this, however, is impossible since $\xi_{\tau}>\xi_{\omega}$.

5. Determination of $\theta_{g}$. We now consider the following algorithm [3, p. $453 \mathrm{ff}$.], which we refer to as Algorithm A. We perform the steps in the indicated order; and we denote by $K_{1}(a, b)$ a matrix of the form $\left(\begin{array}{cc}a & 1 \\ b & 0\end{array}\right)$, by $K_{2}(a, b)$ a matrix of the form $\left(\begin{array}{ll}1 & a \\ 0 & b\end{array}\right)$ and by $K_{3}(a, b)$ a matrix of the form $\left(\begin{array}{ll}0 & a \\ 1 & b\end{array}\right)$.

(i) Transform the basis by $K=\left(\begin{array}{cc}k_{1} & 0 \\ 0 & k_{2}\end{array}\right)$, where $k_{1}=\operatorname{sgn}\left(\xi_{\mu}\right), k_{2}=\operatorname{sgn}\left(\xi_{\nu}\right)$.

(ii) If $\xi_{\nu}<\xi_{\mu}$, transform the basis by $K_{2}(1,1)$ and go to (iii) unless $\eta_{\nu} \eta_{\mu}<0$ and $\left|\eta_{\nu}\right|>\left|\eta_{\mu}\right|$. If this latter case occurs, transform the basis by $K_{1}(0,1)$ and go to $(\mathrm{v})$.

(iii) If $\eta_{\mu} \eta_{\nu}<0$, omit the rest of step (iii) and perform step (iv).

(a) If $\left[\xi_{\nu} / \xi_{\mu}\right]=\left[\eta_{\nu} / \eta_{\mu}\right]=k$, transform the basis by $K_{1}(-k, 1)$ and continue to perform this step until $\left[\xi_{\nu} / \xi_{\mu}\right] \neq\left[\eta_{\nu} / \eta_{\mu}\right]$.

(b) If $\left[\xi_{\nu} / \xi_{\mu}\right]+1=\left[\eta_{\nu} / \eta_{\mu}\right]=k$, transform the basis by $K_{1}(k,-1)$ and go to step (iv).

(c) If $k=\left[\xi_{\nu} / \xi_{\mu}\right]=\left[\eta_{\nu} / \eta_{\mu}\right]+1$, transform the basis by $K_{1}(-k, 1)$ and go to (iv).

(d) If $\left[\xi_{\nu} / \xi_{\mu}\right]<\left[\eta_{\nu} / \eta_{\mu}\right]-1$, transform the basis by either of $K_{1}\left(k_{1},-1\right)$ or $K_{2}\left(k_{2},-1\right)$, where $k_{1}=\left[\xi_{\nu} / \xi_{\mu}\right]+1, k_{2}=\left[\eta_{\nu} / \eta_{\mu}\right]$. Go to $\operatorname{step}(v)$.

(e) If $\left[\xi_{\nu} / \xi_{\mu}\right]>\left[\eta_{\nu} / \eta_{\mu}\right]+1$, transform the basis by either of $K_{1}\left(-k_{1}, 1\right)$ or $K_{2}\left(-k_{2}, 1\right)$, where $k_{1}=\left[\xi_{\nu} / \xi_{\mu}\right], k_{2}=\left[\eta_{\nu} / \eta_{\mu}\right]+1$.

Go to step (v). 
(iv) If $\left|\eta_{\mu}\right|>\left|\eta_{\nu}\right|$ go to (v); otherwise, transform the basis by either of $K_{1}\left(-k_{1}, 1\right)$ or $K_{2}\left(k_{2}, 1\right)$, where $k_{1}=\left[\xi_{\nu} / \xi_{\mu}\right], k_{2}=\left[\left|\eta_{\nu} / \eta_{\mu}\right|\right]$.

(v) At this point $\xi_{\nu}>\xi_{\mu}>0, \eta_{\mu} \eta_{\nu}<0$, and $\left|\eta_{\nu}\right|<\left|\eta_{\mu}\right|$.

(a) If $\left|\eta_{\nu}\right|>1 / 2$ and $\left|\eta_{\mu}\right|<1 / 2$, terminate the algorithm.

(b) If $\left|\eta_{\mu}\right|<1 / 2$, transform the basis by $K_{1}\left(-k_{1}, 1\right)$, where $k_{1}=$ $\left[\xi_{\nu} / \xi_{\mu}\right]$ until $\left|\eta_{\mu}\right|>1 / 2$. At this point the algorithm terminates.

(c) If $\left|\eta_{\nu}\right|>1 / 2$, transform the basis by $K_{3}\left(1, k_{2}\right)$, where $k_{2}=$ $\left[\left|\eta_{\mu}\right| \eta_{\nu} \mid\right]$, until $\left|\eta_{\nu}\right|<1 / 2$. At this point the algorithm terminates.

After this algorithm has terminated we have a new basis $[1, M, N]$ of $R$ and for this basis it is not difficult to show that $\mu, \nu$ form a basis for $L, \xi_{\nu}>\xi_{\mu}>0$, $\eta_{\mu} \eta_{\nu}<0,\left|\eta_{\nu}\right|<1 / 2,\left|\eta_{\mu}\right|>1 / 2$. In the following theorem we assume the existence of $\Phi$ and $\Psi \in R$ such that $[1, \Phi, \Psi]$ is a basis of $R$ and $\xi_{\phi}>\xi_{\psi}>0, \eta_{\phi} \eta_{\psi}<$ $0,\left|\eta_{\phi}\right|<\left|\eta_{\psi}\right|,\left|\eta_{\phi}\right|<1 / 2+\beta,\left|\eta_{\psi}\right|>1 / 3$. Certainly, in view of Algorithm A, such a pair exists. The difficulty occurs in attempting to find them by using an algorithm which uses only a finite amount of precision. We discuss such an algorithm in a later section.

THEOREM 5.1. If $\theta$ is the puncture of $\theta_{g}$, then

$$
\theta=a \phi+b \psi
$$

where $(a, b) \in S=\{(-1,2),(1,-2),(1,0),(0,1),(1,1),(1,-1),(2,1)\}$.

Proof. Clearly, $\theta=a \phi+b \psi$ for some $(a, b) \in Z^{2}$; also $\theta_{g} \in C$ and, therefore, $\left|\eta_{\theta}\right|<1$.

If $a<0$, then $b>0$; also, $\left|\eta_{\theta}\right|=|a|\left|\eta_{\phi}\right|+b\left|\eta_{\psi}\right|<1$. Since $\left|\eta_{\psi}\right|>1 / 3$, it follows that $b \leqslant 2$. Since $\xi_{\theta}=b \xi_{\psi}-|a| \xi_{\phi}>0$, we have $|a|<b$ and, therefore, $(a, b)=(-1,2)$.

If $a=0$, then since $\xi_{\theta}=b \xi_{\psi}$ and $\left|\eta_{\theta}\right|=b\left|\eta_{\psi}\right|$, we see that $0<b<3$. If $b$ $=2$, then $\left|\eta_{\psi}\right|<1 / 2$, and $\eta_{\theta}$ and $\eta_{\psi}$ have the same sign. Also, since $\theta_{g}<\Psi^{*}$ we have a contradiction to Lemma 4.3. Thus, if $a=0$, we can only have $b=1$.

If $a \geqslant 1$ and $b \leqslant 0$, then $\left|\eta_{\theta}\right|=a\left|\eta_{\phi}\right|+|b|\left|\eta_{\psi}\right|<1$; hence, $|b| \leqslant 2$. Suppose $b=-2$; we have $\eta_{\phi}$ and $\eta_{\phi}-\eta_{\psi}$ with the same sign and since $\eta_{\theta}=2\left(\eta_{\phi}-\eta_{\psi}\right)+$ $(a-2) \eta_{\phi}$, we see that, if $a \geqslant 2, \eta_{\theta}$ has the same sign as $\eta_{\phi}-\eta_{\psi}$ and $\left|\eta_{\phi}-\eta_{\psi}\right|<$ $1 / 2$. But $\xi_{\theta}>\xi_{\phi-\psi}$ and $\theta_{g}<(\Phi-\Psi)^{*}$ contradicts Lemma 4.3 ; thus, $(a, b)=$ $(1,-2)$. Suppose next that $b=-1$; then $\eta_{\theta}$ and $\eta_{\phi}$ have the same sign, $\left|\eta_{\phi}\right|<\sqrt{3} / 2$ and, if $a \geqslant 2, \xi_{\theta}>\xi_{\phi}$. This also contradicts Lemma 4.3; hence, $(a, b)=(1,-1)$. If $b=0$, then $a=1$; for, otherwise, we would again contradict Lemma 4.3.

We are left to consider the case of $a, b \geqslant 1$. If $b \geqslant a$, let $b=a+c$, where $c \geqslant$ 0 . Now $\eta_{\phi+\psi}$ and $\eta_{\psi}$ have the same sign and if $\left|\eta_{\phi+\psi}\right|>1 / 2$, we have $\left|\eta_{\psi}\right|>1 / 2$ and $\left|\eta_{\theta}\right|=a\left|\eta_{\phi+\psi}\right|+c\left|\eta_{\psi}\right|>(a+c) / 2 \geqslant 1$ when $c, a \geqslant 2$ or $a=c=1$. As $\eta_{\theta}$ and $\eta_{\phi+\psi}$ have the same sign and $\xi_{\theta}>\xi_{\phi+\psi}$, we cannot have $\left|\eta_{\phi+\psi}\right|<1 / 2$ by Lemma 4.3. We see that $(a, b)=(1,1)$. Finally, we note that if $a>b \geqslant 1$ and $a \geqslant 3$, 
then

$$
\begin{aligned}
\xi_{\theta} & =\xi_{\phi}+(a-1) \xi_{\phi}+b \xi_{\psi}=\xi_{\phi}+\lambda+1, \\
\lambda & =(a-1) \xi_{\phi}+b \xi_{\psi}-1>2 \xi_{\phi}-1>2 \sqrt{1-(1 / 2+\beta)^{2}}-1 \\
& \geqslant g(\beta) .
\end{aligned}
$$

From Lemma 4.5 we must have $a \leqslant 2$ and $(a, b)=(2,1)$.

Corollary 5.1.1. If $\left|\eta_{\psi}\right|>\left(1-\left|\eta_{\phi}\right|\right) / 2$, then $(a, b) \neq(-1,2)$ or $(1,-2)$.

Proof. If $\theta= \pm(\phi-2 \psi)$, then $\left|\eta_{\theta}\right|>1$.

Corollary 5.1.2. If $\xi_{\psi}>.256$, then $(a, b) \neq(2,1)$.

Proof. If $\xi_{\psi}>.256$ and $(a, b)=(2,1)$, then

$$
\xi_{\theta}=2 \xi_{\phi}+\xi_{\psi}=\xi_{\phi}+\lambda+1
$$

where

$$
\lambda=\xi_{\phi}+\xi_{\psi}-1>.256+\sqrt{1-(1 / 2+\beta)^{2}}-1 \geqslant g(\beta) .
$$

Since $\left|\eta_{\phi}\right|<\sqrt{3} / 2$, we cannot have $\theta_{g}<\Phi^{*}$ by Lemma 4.5 .

Corollary 5.1.3. If $\left|\eta_{\psi}\right|<\sqrt{3} / 2$, then $(a, b) \neq(1,1),(2,1)$; if $\left|\eta_{\psi}\right|>3 / 2$ and $\xi_{\psi}<1 / 2$, then $(a, b) \neq(0,1)$.

Proof. If $\left|\eta_{\psi}\right|<\sqrt{3} / 2$, then $\Psi^{*}$ exists. Since $\Phi^{*}$ also exists, $\eta_{\phi} \eta_{\psi}<0$, and $\xi_{\theta}>\xi_{\psi}$, $\xi_{\phi}$ when $\theta=\phi+\psi$ or $\theta=2 \phi+\psi$, we see that $\theta_{g}$ cannot be less than both $\Psi^{*}$ and $\Phi^{*}$ by Lemma 4.3 .

If $\left|\eta_{\psi}\right|>\sqrt{3} / 2$ and $\Psi^{*}$ exists, then $\zeta_{\Psi^{*}}<1 / 2$; hence, if $\xi_{\psi}<1 / 2$, we get $\Psi^{*}$ $=\zeta_{\Psi^{*}}+\xi_{\psi}<1$, which is impossible as 1 is a relative minimum.

The corollaries of Theorem 5.1 allow us to restrict even further the set $S$ from which the possible value of $(a, b)$ can be obtained such that $\theta=a \phi+b \psi$. We summarize these results in Table 2 below. It is assumed in this table that $\left|\eta_{\psi}\right|>$ $\left(1-\left|\eta_{\phi}\right|\right) / 2$.

TABLE 2

\begin{tabular}{lc}
\hline $\begin{array}{c}\text { Restrictions on } \\
\phi \text { and } \psi\end{array}$ & $S$ \\
\hline$\left|\eta_{\psi}\right|<\sqrt{3} / 2$ & $\{(1,0),(0,1),(1,-1)\}$ \\
\hline $\begin{array}{l}\xi_{\psi}>.256 \\
\mid \begin{array}{l}\eta_{\psi} \mid>\sqrt{3} / 2, \\
\xi_{\psi}<1 / 2\end{array}\end{array}$ & $\{(1,0),(0,1),(1,-1),(1,1)\}$ \\
\hline
\end{tabular}


We now have an algorithm to find $\theta_{g}$. We first determine $\Phi$ and $\Psi$, then create the subset made up of those members of the set $\{a \Phi+b \Psi \mid(a, b) \in S\}$ such that $(a \Phi+b \Psi)^{*}$ exists. Since $\left|\eta_{\phi}\right|<\sqrt{3} / 2$, this subset is not empty. Put $\theta_{g}$ equal to that element of this subset such that $(a \Phi+b \Psi)^{*}$ is least. If $\theta_{g}=\Phi^{*}$, put $\theta_{h}$ equal to one of the points which belong to $\Psi$; if $\theta_{g} \neq \Phi^{*}$, put $\theta_{h}=\Phi^{*}$. Since $[1, \Phi, \Psi]$ is a basis of $R$, so is $\left[1, \theta_{g}, \theta_{h}\right]$.

6. Some Useful Inequalities. If $\left[1, M_{r}, N_{r}\right]$ is a basis of $R_{r}$ and $\mu=\left(\xi_{\mu}, \eta_{\mu}\right)$, $\nu=\left(\xi_{\nu}, \eta_{\nu}\right)$ are, respectively, the punctures of $M_{r}$ and $N_{r}$, put $E_{r}=\left|\xi_{\mu} \eta_{\nu}-\xi_{\nu} \eta_{\mu}\right|$. It is a simple matter [6, Section 29] to show that $E_{r}=\left|e_{r}\right| \sqrt{|\Delta|} / 2 \sigma_{r}^{2}$. Since the value of $\left|e_{r}\right|$ is the same for any basis of $R_{r}$, so is the value of $E_{r}$. Voronoi [6, Section 30] also showed that $E_{r}>\sqrt{3} / 2$ and that if $\Theta$ is a relative minimum of the first kind adjacent to 1 in $R_{r}$, then $\Theta^{\prime} \Theta^{\prime \prime}<P+1 / 4$, where $P$ is the minimum of a certain quadratic form $A x^{2}+2 B x y+C y^{2}$, with $A C-B^{2}=E_{r}^{2}[6$, Sections 27, 28]. In this section we make use of these results to find inequalities which will be useful in the following sections.

We first note that when we are dealing with pure cubic fields, $|\Delta|=27 D^{2}$. Since $E_{r}>\sqrt{3} / 2$, we have

$$
\sigma_{r}^{2} /\left|e_{r}\right|<3 D
$$

and consequently, (Theorem 3.1)

$$
\begin{gathered}
\left|e_{r}\right| \leqslant \sigma_{r}<3 D, \\
\sigma_{r} /\left|e_{r}\right|<\sqrt{3 D}, \\
\sigma_{1} Q_{r}<3 D .
\end{gathered}
$$

In several of the following sections we shall be concerned about developing a means of finding a $\beta$-basis of $R_{r}$. This is a basis $[1, \Phi, \Psi]$ of $R_{r}$ such that the punctures $\phi=\left(\xi_{\phi}, \eta_{\phi}\right)$ and $\psi=\left(\xi_{\psi}, \eta_{\psi}\right)$ (of $\Phi$ and $\Psi$, respectively) possess the following properties:

(1) $\xi_{\phi}>\xi_{\psi}>0$

(2) $\left|\eta_{\phi}\right|<\left|\eta_{\psi}\right|, \eta_{\phi} \eta_{\psi}<0$,

(3) $\left|\eta_{\phi}\right|<1 / 2+\beta=1-\sqrt{3} / 4,\left|\eta_{\psi}\right|>1 / 2-\beta=\sqrt{3} / 4$.

(4) $\left|\eta_{\psi}\right|>\left(1-\left|\eta_{\phi}\right|\right) / 2$.

It follows that, since $\left|\eta_{\phi}\right|<\sqrt{3} / 2, \Phi^{*}$ as defined in Section 2 must exist. Further, since $1 / 2-\beta>1 / 3$, by Theorem 5.1 and Corollary 5.1.1, we see that if $\theta$ is the puncture of $\theta_{g}^{(r)}$, then $\theta=a \phi+b \psi$, where $(a, b)$ is one of the elements of the set $\{(1,0)$, $(0,1),(1,1),(1,-1),(2,1)\}$.

In Lemma 6.1 we derive some important inequalities concerning $\beta$-bases when $\delta^{3}$ $=D(D \in Z)$. Indeed, in all the remaining sections of this paper we shall confine our discussion to lattices $R$, where $\delta^{3}=D(D \in Z)$.

LEMma 6.1. Let $\Phi=\left(\xi_{\phi}, \eta_{\phi}\right), \Psi=\left(\xi_{\psi}, \eta_{\psi}\right)$ be the punctures of $\Phi$ and $\Psi$ respectively, where $[1, \Phi, \Psi]$ is a $\beta$-basis of $R_{r}$. If $\Phi^{*}=\left(\bar{s}_{1}+s_{2} \delta+s_{3} \delta^{2}\right) / \sigma_{r}$ and $\Psi_{i}$ $=\left(\bar{t}_{1}+t_{2} \delta+t_{3} \delta^{2}\right) / \sigma_{r}(i=1$ or 2$)$ is either of the two points of $R_{r}$ which belongs 
to $\Psi$, then

$$
0<t_{2}+t_{3} \delta<s_{2}+\delta s_{3}<4 \delta^{2}, \quad\left|s_{2}-\delta s_{3}\right|<2 \delta^{2}, \quad\left|t_{2}-t_{3} \delta\right|<3.65 \delta^{2}
$$

and

$$
\left|s_{2}\right|,\left|\delta s_{3}\right|<3 \delta^{2}, \quad\left|t_{2}\right|,\left|\delta t_{3}\right|<3.83 \delta^{2} ; \quad\left|\bar{s}_{1}\right|,\left|\bar{t}_{1}\right|<5 D
$$

Proof. We first notice that

$$
E_{r}=\xi_{\phi}\left|\eta_{\psi}\right|+\left|\eta_{\phi}\right| \xi_{\psi}
$$

hence, $\xi_{\phi}\left|\eta_{\psi}\right|,\left|\eta_{\phi}\right| \xi_{\psi}<E_{r}$. Also, since $\left|\eta_{\phi}\right|<\sqrt{3} / 2$, we must have $\xi_{\phi}>\sqrt{1-\eta_{\phi}^{2}}$ by Lemma 4.2 . Thus,

$$
\sqrt{1-\eta_{\phi}^{2}}<\xi_{\phi}<E_{r} /\left|\eta_{\psi}\right|, \quad 0<\xi_{\psi}<\xi_{\phi},
$$

and

$$
\left|\eta_{\phi}\right|<\left|\eta_{\psi}\right|<E_{r} /\left|\xi_{\phi}\right|<E_{r} / \sqrt{1-\eta_{\phi}^{2}}
$$

Now $\left|\eta_{\psi}\right|>\sqrt{3} / 4 ;$ hence,

$$
\xi_{\phi}=\frac{3 \delta\left(s_{2}+s_{3} \delta\right)}{2 \sigma_{r}}<\frac{4}{\sqrt{3}} E_{r}=\frac{6\left|e_{r}\right| D}{\sigma_{r}^{2}}
$$

and

$$
0<s_{2}+s_{3} \delta<4\left|e_{r}\right| D / \sigma_{r} \delta \leqslant 4 \delta^{2} \quad(\text { by }(6.2))
$$

Also,

consequently,

$$
\left|\eta_{\phi}\right|=\frac{\sqrt{3} \delta\left|s_{2}-s_{3} \delta\right|}{2 \sigma_{r}}<1-\sqrt{3} / 4
$$

$$
\left|s_{2}-s_{3} \delta\right|<\frac{2(1-\sqrt{3} / 4) \sigma_{r}}{\sqrt{3} \delta}<2 \delta^{2} .
$$

It follows from the results on $s_{2}+s_{3} \delta$ and $\left|s_{2}-s_{3} \delta\right|$ that $\left|s_{2}\right|,\left|\delta s_{3}\right|<3 \delta^{2}$.

Since $\eta_{\phi}<1-\sqrt{3} / 4$, we have $\sqrt{1-\eta_{\phi}^{2}}>\sqrt{\sqrt{3} / 2-3 / 16}$. The results involving $t_{2}$ and $t_{3}$ can be easily derived from the inequalities

$$
\xi_{\psi}<\xi_{\phi} \text { and }\left|\eta_{\psi}\right|<E_{r} / \sqrt{\sqrt{3} / 2-3 / 16}
$$

Clearly, $\left|\zeta_{\Psi_{i}}\right|,\left|\zeta_{\Phi^{*}}\right|<1$ and $0<\xi_{\psi}, \xi_{\phi}<2$; thus, since $\bar{s}_{1} / \sigma_{r}=\zeta_{\Phi^{*}}+\xi_{\phi} / 3$, we get $\left|\bar{s}_{1}\right|<5 D$. Similarly, $\left|\bar{t}_{1}\right|<5 D$.

LEMMA 6.2. If $\Theta=\theta_{g}^{(r)}=\left(m_{1}+m_{2} \delta+m_{3} \delta^{2}\right) / \sigma_{r}$ is the relative minimum of the second kind adjacent to 1 in $R_{r}$, then

$$
\left|m_{2}\right|+\left|m_{3}\right| \delta<(2+2 \sqrt{3}) \delta^{2}
$$


and

$$
-3 D<m_{1}<(\sqrt{3}+5 / 2) D ; \quad\left|m_{2}\right|,\left|\delta m_{3}\right|<(1+2 \sqrt{3}) \delta^{2} .
$$

Proof. By the construction technique of Algorithm A we know there exists a $\beta$ basis $[1, \Phi, \Psi]$ such that $\left|\eta_{\phi}\right|<1 / 2$ and $\left|\eta_{\psi}\right|>1 / 2$. If we refer to Lemma 4.5 with $\kappa=0=g(0)$, we see that $\xi_{\theta}<\xi_{\phi}+1$. For, if $\xi_{\theta} \geqslant \xi_{\phi}+1$, then $\xi_{\theta}=1+\lambda+\xi_{\phi}$, where $\lambda=\xi_{\theta}-\xi_{\phi}-1 \geqslant 0=g(0)$. Hence, from Lemma 6.1 we get

$$
0<\frac{3 \delta\left(m_{2}+m_{3} \delta\right)}{2 \sigma_{r}}<2 E_{r}+1=\frac{3 \sqrt{3}\left|e_{r}\right| D}{\sigma_{r}^{2}}+1
$$

and since $\left|\eta_{\theta}\right|<1$, we also get

$$
\left|\frac{\sqrt{3} \delta\left(m_{2}-m_{3} \delta\right)}{2 \sigma_{r}}\right|<1
$$

Thus, $\left|m_{2}+m_{3} \delta\right|<2(\sqrt{3}+1) \delta^{2}$ and $\left|m_{2}-m_{3} \delta\right|<2 \sqrt{3} \delta^{2}$. It follows that $\left|m_{2}\right|+$ $\left|m_{3}\right| \delta<(2+2 \sqrt{3}) \delta^{2}$ and $\left|m_{2}\right|,\left|\delta m_{3}\right|<(1+2 \sqrt{3}) \delta^{2}$. Since (Lemma 4.1) $-1<\xi_{\Theta}<1 / 2$, we have $-1+\xi_{\theta} / 3<m_{1} / \sigma_{r}<1 / 2+\xi_{\theta} / 3$; thus $-\sigma_{r}<m_{1}<5 \sigma_{r} / 6$ $+\sqrt{3}\left|e_{r}\right| D / \sigma_{r}$ and the result for $m_{1}$ follows.

We now give two very simple lemmas which will be needed in the proofs of the last three lemmas of this section.

LeMmA 6.3. If $x^{2}+y^{2}=a$ and $c>0$, then $|x|+c|y| \leqslant \sqrt{\left(c^{2}+1\right) a}$.

Proof. Since $2 c|x y| \leqslant c^{2} x^{2}+y^{2}$, we have

$$
(|x|+c|y|)^{2}=x^{2}+2 c|x y|+c^{2} y^{2} \leqslant\left(c^{2}+1\right)\left(x^{2}+y^{2}\right)=\left(c^{2}+1\right) a ;
$$

hence, $|x|+c|y| \leqslant \sqrt{\left(c^{2}+1\right) a}$.

LEMMA 6.4. If $x^{2}+y^{2}=a$ and $b>1$, then

$$
1+2|x|+x^{2}+b y^{2}<1+a b+(b-1)^{-1} .
$$

Proof. This result follows from the fact that $(1+(1-b)|x|)^{2}>0$.

LEMMA 6.5. If $M_{r}=1 / \theta_{g}^{(r-1)}=\left(m_{1}^{*}+m_{2}^{*} \delta+m_{3}^{*} \delta^{2}\right) / \sigma_{r}$, then

$$
\left|m_{2}^{*}+m_{3}^{*} \delta\right|<\sqrt{6\left|e_{r}\right| \delta}, \quad\left|m_{2}^{*}-m_{3} \delta\right|<\sqrt{5\left|e_{r}\right| \delta}
$$

and

$$
-\sqrt{5} D<m_{1}^{*}<(1+\sqrt{5}) D ; \quad\left|m_{2}^{*}\right|,\left|m_{3}^{*} \delta\right|<(1+\sqrt{5}) \delta^{2} .
$$

Proof. By Theorem 2.1, $M_{r}=1 / \theta_{g}^{(r-1)}>0$ is the relative minimum of the first kind adjacent to 1 . Since it is well known that the minimum $P$ of a quadratic form $A x^{2}+2 B x y+C y^{2}$ does not exceed $2 \sqrt{A C-B^{2}} / \sqrt{3}$, we have

$$
M_{r}^{\prime} M_{r}^{\prime \prime}<\frac{2}{\sqrt{3}} E_{r}+1 / 4
$$

by the remark at the beginning of this section. Thus, if $\left(\xi_{\mu}, \eta_{\mu}\right)$ is the puncture of 
$M_{r}$, then

$$
\zeta_{M_{r}}^{2}+\eta_{\mu}^{2}<\frac{2}{\sqrt{3}} E_{r}+1 / 4
$$

and since $M_{r}=\zeta_{M_{r}}+\xi_{\mu}<1$, we get

$$
\left|\zeta_{M_{r}}\right|,\left|\eta_{\mu}\right|<\sqrt{\frac{2}{\sqrt{3}} E_{r}+\frac{1}{4}}, \quad\left|\xi_{\mu}\right|<\sqrt{\frac{2}{\sqrt{3}} E_{r}+\frac{1}{4}}+1 .
$$

Since $E_{r}=3 \sqrt{3} D\left|e_{r}\right| / 2 \sigma_{r}^{2}$, we have, on using (6.1),

hence,

$$
\left|\eta_{\mu}\right|<\frac{\sqrt{15 D\left|e_{r}\right|}}{2 \sigma_{r}}, \quad\left|\xi_{\mu}\right|<\frac{\sqrt{15 D\left|e_{r}\right|}}{2 \sigma_{r}}+1
$$

$$
\left|m_{2}^{*}-m_{3}^{*} \delta\right|<\sqrt{5\left|e_{r}\right| \delta}, \quad\left|m_{2}^{*}+m_{3}^{*} \delta\right|<\sqrt{6\left|e_{r}\right| \delta}
$$

Since $0<M_{r}<1$ and $\left|\zeta_{M_{r}}\right|<\sqrt{(2 / \sqrt{3}) E_{r}+1 / 4}$, we have

$$
0<m_{1}^{*}+m_{2}^{*} \delta+m_{3}^{*} \delta^{2}<\sigma_{r}, \quad\left|2 m_{1}^{*}-\delta\left(m_{2}^{*}+\delta m_{3}^{*}\right)\right|<2 \sigma_{r} \sqrt{\frac{2}{\sqrt{3}} E_{r}+1 / 4}
$$

thus, from (6.2), we get $-\sqrt{5} D<m_{1}^{*}<(1+\sqrt{5}) D$.

Since

$$
m_{2}^{*}=\frac{\sigma_{r}}{3 \delta}\left(\xi_{\mu}+\sqrt{3} \eta_{\mu}\right), \quad \delta m_{3}^{*}=\frac{\sigma_{r}}{3 \delta}\left(\xi_{\mu}-\sqrt{3} \eta_{\mu}\right) \quad \text { and } \quad\left|\xi_{\mu}\right|<1+\left|\zeta_{M_{r}}\right|
$$

we have

$$
\left|m_{2}^{*}\right|,\left|\delta m_{3}^{*}\right|<\frac{\sigma_{r}}{3 \delta}\left(1+2 \sqrt{\frac{2}{\sqrt{3}} E_{r}+1 / 4}\right)<(1+\sqrt{5}) \delta^{2}
$$

from Lemma 6.3.

From (3.2), (6.3) and the results of the above lemma, we get

$$
\left(\delta^{i-1} m_{i}^{\prime}\right) /\left|e_{r-1}\right|<(1+\sqrt{5}) \sqrt{3} D^{3 / 2} \quad(i=1,2,3) .
$$

Also, by using the above results, we see that $\xi_{\mu}+c\left|\eta_{\mu}\right|<1+\left|\zeta_{M_{r}}\right|+c\left|\eta_{\mu}\right|$, where

$$
\zeta_{M_{r}}^{2}+\eta_{\mu}^{2}<\frac{2}{\sqrt{3}} E_{r}+1 / 4<45 D^{2} / 4 \sigma_{r}^{2}
$$

hence,

$$
\left|\xi_{\mu}\right|+c\left|\eta_{\mu}\right|<1+3 D \sqrt{5\left(c^{2}+1\right)} / 2 \sigma_{r} \quad(c>0) .
$$

Another result of this type is given in

Lemma 6.6. If $(c, d) \in\{(1,9),(1 / 3,27),(9,1),(3,3)\}$, then $c \xi_{\mu}^{2}+d \eta_{\mu}^{2}<$ $307 D^{2} / \sigma_{r}^{2}$. 
Proof. When $d / c \leqslant 1$, this result follows on using the results of Lemma 6.5. When $d / c>1$, we use Lemma 6.4 to show that

$$
\begin{aligned}
c \xi_{\mu}^{2}+d \eta_{\mu}^{2} & =c\left(\xi_{\mu}^{2}+\eta_{\mu}^{2} d / c\right)<c\left(1+2\left|\xi_{M_{r}}\right|+\zeta_{M_{r}}^{2}+\eta_{\mu}^{2} d / c\right) \\
& <c\left(1+\left(\frac{3\left|e_{r}\right| \delta^{3}}{\sigma_{r}^{2}}+1 / 4\right) \frac{d}{c}+\frac{c}{d-c}\right) \\
& <307 D^{2} / \sigma_{r}^{2} .
\end{aligned}
$$

Let $\left[1, M_{r}, N_{r}\right]$ be a basis of $R_{r}$, where

$$
\begin{aligned}
& M_{r}=1 / \theta_{g}^{(r-1)}=\left(m_{1}^{*}+m_{2}^{*} \delta+m_{3}^{*} \delta^{2}\right) / \sigma_{r}, \\
& N_{r}=\theta_{h}^{(r-1)} / \theta_{g}^{(r-1)}=\left(n_{1}^{*}+n_{2}^{*} \delta+n_{3}^{*} \delta^{2}\right) / \sigma_{r} ;
\end{aligned}
$$

and let $[1, \bar{M}, \bar{N}]$ be the basis formed by transforming $\left[1, \bar{M}_{r}, \bar{N}_{r}\right]$ by $K$, where $K=$ $K_{1}(-k, 1)$ and

$$
k= \begin{cases}{\left[n_{3}^{*} / m_{3}^{*}\right]} & \text { when } m_{3}^{*} \neq 0 \\ {\left[n_{2}^{*} / m_{2}^{*}\right]} & \text { when } m_{3}^{*}=0\end{cases}
$$

Our final lemma of this section is

LEMMA 6.7. If $\bar{M}=\left(\bar{m}_{1}+\bar{m}_{2} \delta+\bar{m}_{3} \delta^{2}\right) / \sigma_{r}$ and $\bar{N}=\left(\bar{n}_{1}+\bar{n}_{2} \delta+\bar{n}_{3} \delta^{2}\right) / \sigma_{r}$, then

$$
\left|\bar{n}_{2}\right|<(1+\sqrt{5}) \delta^{2}+\left|e_{r}\right| \text { and }\left|\bar{m}_{2}\right|, \delta\left|\bar{m}_{3}\right|, \delta\left|\bar{n}_{3}\right|<(1+\sqrt{5}) \delta^{2} \text {. }
$$

Proof. From (3.3), we see that if $m_{3}^{*} \neq 0$, then

$$
\bar{m}_{2}=m_{2}^{*}, \quad \bar{m}_{3}=m_{3}^{*}, \quad\left|\bar{n}_{3}\right|<\left|\bar{m}_{3}\right|, \quad\left|\bar{n}_{2}\right|<\left|e_{r}\right| /\left|m_{3}^{*}\right|+\left|m_{2}^{*}\right|
$$

In this case the lemma follows easily from Lemma 6.5. If $m_{3}^{*}=0$, then $\bar{m}_{2}=m_{2}^{*}$, $\bar{m}_{3}=0,\left|\bar{n}_{2}\right|<\left|\bar{m}_{2}\right|$. Also, since $[1, \bar{M}, \bar{N}]$ is a basis of $R_{r}$, and $[1, \Phi, \Psi]$ is a basis of $R_{r}$, there must exist a matrix $K=\left(k_{i j}\right)_{2 \times 2}$ with integer coefficients such that $|K|= \pm 1$ and

$$
\left(\begin{array}{ll}
s_{2} & t_{2} \\
s_{3} & t_{3}
\end{array}\right)=\left(\begin{array}{ll}
\bar{m}_{2} & \bar{n}_{2} \\
\bar{m}_{3} & \bar{n}_{3}
\end{array}\right) K .
$$

Since $\bar{m}_{3}=0$, we have $s_{3}=k_{21} \bar{n}_{3}$ and by Lemma $6.1,\left|\bar{n}_{3}\right|<3 \delta$. We also have $\left|\bar{n}_{2}\right|<\left|\bar{m}_{2}\right|<(1+\sqrt{5}) \delta^{2}$ by Lemma 6.5 .

We conclude this section with a summary of the several inequalities derived here. We give this as Table 3 . 
TABLE 3

Description of Symbols

Number

Inequalities

(6.1) $\quad \sigma_{r}^{2} /\left|e_{r}\right|<3 D$

See Table 1.

(6.2) $\quad\left|e_{r}\right| \leqslant \sigma_{r}<3 D$

(6.3) $\quad \sigma_{r} /\left|e_{r}\right|<\sqrt{3 D}$

(6.4) $\quad \sigma_{1} Q_{r}<3 D$

$\left(\bar{s}_{1}+s_{2} \delta+s_{3} \delta^{2}\right) \sigma_{r}=\Phi$,

(6.5) $0<t_{2}+t_{3} \delta<s_{2}+\delta s_{3}<4 \delta^{2}$

$\left|s_{2}-\delta s_{3}\right|<2 \delta^{2} ;\left|t_{2}-t_{3} \delta\right|<3.65 \delta^{2}$

$\left(\bar{t}_{1}+t_{2} \delta+t_{3} \delta^{2}\right) / \sigma_{r}=\Psi_{i}$, where $[1, \Phi, \Psi]$ is a $\beta$-basis of $R_{r}$ and $\Psi_{i}(i=1$ or 2$)$ is either of the points which belong to the puncture of $\Psi$

(6.6) $\left|s_{2}\right|, \delta\left|s_{3}\right|<3 \delta^{2} ;\left|t_{2}\right|,\left|\delta t_{3}\right|<3.83 \delta^{2}$

(6.7) $\quad\left|\bar{s}_{1}\right|,\left|\bar{t}_{1}\right|<5 D$

(6.8) $\quad\left|m_{2}\right|+\left|m_{3}\right| \delta<(2+2 \sqrt{3}) \delta^{2}$

$$
\theta_{g}^{(r)}=\left(m_{1}+m_{2} \delta+m_{3} \delta^{2}\right) / \sigma_{r} \quad \begin{array}{ll}
-3 D<m_{1}<(\sqrt{3}+5 / 2) D, \\
& \left|m_{2}\right|, \delta\left|m_{3}\right|<(1+2 \sqrt{3}) \delta^{2}
\end{array}
$$

$$
\begin{aligned}
& 1 / \theta_{g}^{(r-1)}= \\
& \left(m_{1}^{*}+m_{2}^{*}+m_{3}^{*} \delta^{2}\right) / \sigma_{r}
\end{aligned}
$$$$
\text { (6.10) } \quad \begin{aligned}
& \left|m_{2}^{*}+m_{3}^{*} \delta\right|<\cdot \sqrt{6\left|e_{r}\right| \delta} \\
& \left|m_{2}^{*}-m_{3}^{*} \delta\right|<\sqrt{5\left|e_{r}\right| \delta}
\end{aligned}
$$$$
\text { (6.11) }-\sqrt{5} D<m_{1}^{*}<(1+\sqrt{5}) D
$$$$
\text { (6.12) }\left|m_{2}^{*}\right|,\left|m_{3}^{*}\right| \delta<(1+\sqrt{5}) \delta^{2}
$$

$$
\begin{aligned}
& m_{1}^{\prime}=m_{1}^{2}-D m_{1} m_{2} \\
& m_{2}^{\prime}=D m_{3}^{2}-m_{1} m_{2} \\
& m_{3}^{\prime}=m_{2}^{2}-m_{1} m_{2},
\end{aligned}
$$

$$
\delta^{i-1} m_{i}^{\prime}|| e_{r} \mid<(1+\sqrt{5}) \sqrt{3} D^{3 / 2},
$$
where $i=1,2,3$.

where $\theta_{g}^{(r)}=$

$\left(m_{1}+m_{2} \delta+m_{3} \delta^{2}\right) / \sigma_{r}$ 
Description of Symbols

Number

Inequalities

$\left(\xi_{\mu}, \eta_{\mu}\right)$ is the

puncture of $1 / \theta_{g}^{(r-1)}$
(6.14) $\quad\left|\xi_{\mu}\right|+c\left|\eta_{\mu}\right|$

$<1+3 D \sqrt{5\left(c^{2}+1\right)} / 2 \sigma_{r}(c<0)$

(6.15) $\quad c \xi_{\mu}^{2}+d \eta_{\mu}^{2}<307 D^{2} / \sigma_{r}$

where

$(c, d) \in\{(1,9),(1 / 3,27),(9,1),(3,3)\}$

$[1, \bar{M}, \bar{N}]$ is the basis of $R_{r}$

formed by transforming

$\left[1,1 / \theta_{g}^{(r-1)}, \theta_{h}^{(r-1)} / \theta_{g}^{(r-1)}\right]$

$$
\begin{aligned}
& \left|\bar{n}_{2}\right|<(1+\sqrt{5}) \delta^{2}+\left|e_{r}\right| \\
& \left|\bar{m}_{2}\right|, \delta\left|\bar{m}_{3}\right|, \delta\left|\bar{n}_{3}\right|<(1+\sqrt{5}) \delta^{2}
\end{aligned}
$$

by $K_{2}(-k, 1)$, where

$k=\left\{\begin{array}{l}{\left[n_{3}^{*} / m_{3}^{*}\right] \text { when } m_{3}^{*} \neq 0} \\ {\left[n_{2}^{*} / m_{2}^{*}\right] \text { when } m_{3}^{*}=0}\end{array}\right.$

7. Modifications of Steps (i)-(iv) of Algorithm A. The object of this section is to develop an algorithm (Algorithm B) to find $P, \Lambda \in R_{r}$ such that $[1, \Lambda, P]$ is a basis of $R_{r}$ and such that if $\left(\xi_{\lambda}, \eta_{\lambda}\right)$ is the puncture of $\Lambda$ and $\left(\xi_{\rho}, \eta_{\rho}\right)$ is the puncture of $P$, then

$$
\xi_{\rho}>\xi_{\lambda}>0 \text { and }\left|\eta_{\rho}\right|<\left|\eta_{\lambda}\right|, \quad \eta_{\lambda} \eta_{\rho}<0 .
$$

That is, we are searching for a basis of $R_{r}$ which satisfies properties (1) and (2) of a $\beta$-basis. We can certainly find such a basis by using steps (i)-(iv) of Algorithm A; however, on a computer this algorithm must make use of approximations which may not be of sufficient accuracy to guarantee a correct answer. We show here how these steps can be modified such that only rational integer arithmetic is needed at any point. In order to do this we first require several lemmas.

LEMMA 7.1. If $r, s \in Z$ and at least one of $r$ and $s$ is not zero, then

$$
|r+s \delta| \geqslant \frac{1}{r^{2}+\delta|r s|+\delta^{2} s^{2}} \geqslant \frac{1}{(|r|+|s| \delta)^{2}} .
$$

Proof. Follows easily from the fact that $r^{3}+s^{3} D$ is a rational nonzero integer.

Define $f(x, y)=x^{2}+\delta|x y|+\delta^{2} y^{2}$. We now prove

LEMMA 7.2. If $r, s, t \in Z, D \geqslant 12$, and $s$, $t$ are not both zero, then

$$
\left|r+s \delta+t \delta^{2}\right|>\frac{1}{3.1 \delta^{2} f(s, t)} .
$$

Proof. Put $A=r+s \delta+t \delta^{2}, \alpha_{1}=A-r, \alpha_{2}=A^{\prime}-r, \alpha_{3}=A^{\prime \prime}-r$. Since

$$
|N(A)|=\left|A A^{\prime} A^{\prime \prime}\right|=|A|\left|A^{\prime}\right|^{2} \geqslant 1
$$


we have

$$
|A| \geqslant 1 /\left|A^{\prime}\right|^{2}
$$

Now if $\gamma=\left|\alpha_{1}-\alpha_{2}\right|$, we have

$$
\gamma^{2}=3 \delta^{2}\left(s^{2}+s t \delta+t^{2} \delta^{2}\right)>15.72
$$

hence,

$$
\gamma+\frac{3}{3.1 \gamma^{2}}<\gamma \sqrt{\frac{3.1}{3}}
$$

Also, $\left|A^{\prime}\right| \leqslant|A|+\gamma$; thus, if we assume that $|A| \leqslant 3 / 3.1 \gamma^{2}$, then $\left|A^{\prime}\right| \leqslant \gamma+3 / 3.1 \gamma^{2}$ and $|A| \geqslant 1 /\left|A^{\prime}\right|^{2}>3 / 3.1 \gamma^{2}$. Since this contradicts our assumption, we must have $|A|>3 / 3.1 \gamma^{2}$.

Corollary 7.2.1. If $r, s \in Z, s \neq 0$, and $D>12$, then $|r+s \delta|>1 / 3.1 \delta^{2} s^{2}$.

Lemma 7.3. If $r, s, t, D$ are as defined in Lemma 7.1, then $r+s \delta+t \delta^{2}$ and $r I+s[\delta I]+t\left[\delta^{2} I\right]$ have the same sign when $I>3.1 \delta^{2}(|s|+|t|) f(s, t)$.

Proof. Let $A=I r+s[\delta I]+t\left[\delta^{2} I\right]$ and $T=I\left(r+s \delta+t \delta^{2}\right)$. By Lemma 7.2, $|T|>|s|+|t|>|A-T|$; hence, $A$ and $T$ have the same sign and the lemma follows.

LEMMA 7.4. If $r, s \in Z, s \neq 0, D>12$ and $I>\min \left\{3.1|s|^{3} \delta^{2},|s| f(r, s)\right\}$, then $r+s \delta$ and $I r+s[\delta I]$ have the same sign.

Proof. By Corollary 7.3.1 and Lemma 7.1,

$$
|I(r+s \delta)|>|s|>|I(r+s \delta)-(I r+s[I \delta])|
$$

Hence, by the same reasoning as that used in Lemma 7.3, we have our result.

For the remainder of the results in this paper we assume that $D \geqslant 12$.

Lemma 7.5. If $r, s, t, u \in Z, t+u \delta \neq 0, k=[(r+s \delta) /(t+u \delta)], r^{\prime}=r-k t$, $s^{\prime}=s-k u,|u|,\left|s^{\prime}\right|,\left|s^{\prime}-u\right|<c_{1}$, and $f(t, u), f\left(r^{\prime}, s^{\prime}\right), f\left(r^{\prime}-t, s^{\prime}-u\right)<c_{2}$, then

$$
k=\left[\frac{I r+s[\delta I]}{I t+u[\delta I]}\right] \text { when } I>\min \left\{c_{1} c_{2}, 3.1 c_{1}^{3} \delta^{2}\right\}
$$

Proof. The lemma is true when $r+s \delta$ is a rational integer multiple of $t+u \delta$. Assume that this is not the case and that $t+u \delta>0$; by Lemma 7.4, It $+u[\delta I]>0$. Now

$$
r^{\prime}+s^{\prime} \delta>0 \text { and } r^{\prime}-t+\left(s^{\prime}-u\right) \delta<0
$$

thus, by Lemma 7.4

$$
I r^{\prime}+s^{\prime}[I \delta]>0 \text { and }\left(r^{\prime}-t\right) I+\left(s^{\prime}-u\right)[\delta I]<0 .
$$


An easy computation gives

$$
\frac{I r+[I \delta] s}{I t+[I \delta] u}-1<k<\frac{I r+[I \delta] s}{I t+[I \delta] u}
$$

and we have the result above. If $t+u \delta<0$, the proof is similar.

Corollary 7.5.1. If $r, s, t, u \in Z, t+u \delta \neq 0, k=[(r+s \delta) /(t+u \delta)], d=$ ts $-r u,|u|<c_{1},|t+u \delta|>c_{3}, I>3.1 \delta^{2}\left(|d| / c_{3}+c_{1}\right)^{3}$, then

$$
k=\left[\frac{I r+s[\delta I]}{I t+u[\delta I]}\right] \text {. }
$$

Proof. Put $s^{\prime}=s-k u$. We first note that

If $u>0$, then

$$
\frac{r+s \delta}{t+u \delta}=\frac{s}{u}-\frac{d}{u(t+u \delta)}
$$

$$
\frac{d}{t+u \delta}<s^{\prime}<\frac{d}{t+u \delta}+u
$$

and if $u<0$, then

$$
\frac{d}{t+u \delta}+u<s^{\prime}<\frac{d}{t+u \delta} .
$$

Thus, $|u|,\left|s^{\prime}\right|,\left|s^{\prime}-u\right|<|d| /|t+u \delta|+|u|<|d| / d_{3}+c_{1}$, and the corollary follows from the lemma.

We are now ready to begin describing how to modify Algorithm A. We first note that by (6.16), we may assume the existence of a basis $[1, M, N]$ of $R_{r}$ such that if $\mu=\left(\xi_{\mu}, \eta_{\mu}\right), \nu=\left(\xi_{\nu}, \eta_{\nu}\right)$ are the punctures of $M$ and $N$, then $M=1 / \theta_{g}^{(r-1)}$,

$$
\begin{array}{rlrl}
\xi_{\mu} & =\frac{3 \delta}{2 \sigma_{r}}\left(m_{2}+\delta m_{3}\right), & \eta_{\mu}=\frac{\sqrt{3} \delta}{2 \sigma_{r}}\left(m_{2}-\delta m_{3}\right), \\
\xi_{\nu}=\frac{3 \delta}{2 \sigma_{r}}\left(n_{2}+\delta n_{3}\right), & \eta_{\nu}=\frac{\sqrt{3} \delta}{2 \sigma_{r}}\left(n_{2}-\delta n_{3}\right),
\end{array}
$$

and

$$
\left|n_{2}\right|<(1+\sqrt{5}) \delta^{2}+\left|e_{r}\right|, \quad\left|m_{2}\right|,\left|\delta m_{3}\right|,\left|\delta n_{3}\right|<(1+\sqrt{5}) \delta^{2} .
$$

We must also define the integers that will be used in Algorithm $B_{1}$ below. Let any $\Omega=\left(q_{1}+q_{2} \delta+q_{3} \delta^{2}\right) / \sigma_{r} \in R_{r}$ have puncture $\omega=\left(\xi_{\omega}, \eta_{\omega}\right)$. We define

$$
x_{\omega}=I_{1} q_{2}+\left[I_{1} \delta\right] q_{3}, \quad y_{\omega}=I_{1} q_{2}-\left[I_{1} \delta\right] q_{3}, \quad \bar{y}_{\omega}=\left[I_{2} \sqrt{3} \delta\right] q_{2}-\left[I_{2} \sqrt{3} \delta^{2}\right] q_{3},
$$

where $I_{1}, I_{2}$ are arbitrary but fixed rational integers. Since

$$
\xi_{\omega}=\frac{3 \delta}{2 \sigma_{r}}\left(q_{2}+q_{3} \delta\right) \text { and } \eta_{\omega}=\frac{\sqrt{3} \delta}{2 \sigma_{r}}\left(q_{2}-q_{3} \delta\right),
$$


we see that

$$
\begin{aligned}
& \left|\frac{\xi_{\omega}}{\xi_{\pi}}-\frac{x_{\omega}}{x_{\pi}}\right|=\frac{\left|e_{r}\right|\left(\delta I_{1}-\left[\delta I_{1}\right]\right)}{\left|x_{\pi}\left(p_{2}+p_{3} \delta\right)\right|} \\
& \left|\frac{\eta_{\omega}}{\eta_{\pi}}-\frac{y_{\omega}}{y_{\pi}}\right|=\frac{\left|e_{r}\right|\left(\delta I_{1}-\left[\delta I_{1}\right]\right)}{\left|y_{\pi}\left(p_{2}-p_{3} \delta\right)\right|} \\
& \left|\frac{x_{\omega}}{x_{\pi}}-\frac{y_{\omega}}{y_{\pi}}\right|=\frac{2 I_{1}\left[I_{1} \delta\right]\left|e_{r}\right|}{\left|I_{1}^{2} p_{2}^{2}-p_{3}^{2}\left[I_{1} \delta\right]^{2}\right|},
\end{aligned}
$$

where $\pi=\left(\xi_{\pi}, \eta_{\pi}\right)$ is the puncture of $\Pi=\left(p_{1}+p_{2} \delta+p_{3} \delta^{2}\right) / \sigma_{r}$. We also have the following simple lemma.

LеммA 7.6. Let $\omega$ be the puncture of $\Omega=\left(q_{1}+q_{2} \delta+q_{3} \delta^{2}\right) / \sigma_{r} \in R_{r}$, where $\left|q_{2}\right|+\left|q_{3}\right|<(3+2 \sqrt{5}) \delta^{2}$. If $I_{2}>80 \delta^{2}$ and $\left|\bar{y}_{\omega}\right| \leqslant I_{2} \sigma_{r}$, we must have $\left|\eta_{\omega}\right|<1-\sqrt{3} / 4$ and if $\left|\bar{y}_{\omega}\right| \geqslant I_{2} \sigma_{r}$, we must have $\left|\eta_{\omega}\right|>\sqrt{3} / 4$.

Proof. We note that

Thus,

$$
\left|\eta_{\omega}-\frac{\bar{y}_{\omega}}{2 \sigma_{r} I_{2}}\right|<\frac{\left|q_{2}\right|+\left|q_{3}\right|}{2 \sigma_{r_{2}}}<\frac{(3+2 \sqrt{5}) \delta^{2}}{2 \sigma_{r_{2}} I_{2}} .
$$

$$
\left|\eta_{\omega}\right|=\left|\frac{\bar{y}_{\omega}}{2 \sigma_{r} I_{2}}\right|+\gamma, \quad \text { where }|\gamma|<\frac{(3+2 \sqrt{5}) \delta^{2}}{2 \sigma_{r} I_{2}}<\frac{1}{2}-\frac{\sqrt{3}}{4}
$$

and the lemma follows.

LEMmA 7.7. With $I_{1}>3.1(2+2 \sqrt{5})^{3} \delta^{5} \simeq 840.44 \delta^{5}$ and $\mu$ and $\nu$ defined as above, we can replace $\xi_{\mu}$ and $\xi_{\nu}$ by $x_{\mu}$ and $x_{\nu}$ in steps (i) and (ii) of Algorithm A.

Proof. By Lemma 7.4 and (7.1) we see that $\operatorname{sgn}\left(x_{\mu}\right)=\operatorname{sgn}\left(\xi_{\mu}\right), \operatorname{sgn}\left(x_{\nu}\right)=$ $\operatorname{sgn}\left(\xi_{\nu}\right), \operatorname{sgn}\left(y_{\mu}\right)=\operatorname{sgn}\left(\eta_{\mu}\right)$, and $\operatorname{sgn}\left(y_{\nu}\right)=\operatorname{sgn}\left(\eta_{\nu}\right)$. Also, if $r=\left|m_{3} \pm n_{3}\right|$, then $r<$ $2(\sqrt{5}+1) \delta$ by $(7.1)$ and consequently, $\operatorname{sgn}\left(\xi_{\nu}-\xi_{\mu}\right)=\operatorname{sgn}\left(x_{\nu}-x_{\mu}\right), \operatorname{sgn}\left(\left|\eta_{\nu}\right|-\left|\eta_{\mu}\right|\right)$ $=\operatorname{sgn}\left(\left|y_{\nu}\right|-\left|y_{\mu}\right|\right)$ by Lemma 7.4.

We have seen that we can replace $\xi_{\mu}$ and $\xi_{\nu}$ by $x_{\mu}$ and $x_{\nu}$ in steps (i) and (ii) of Algorithm $A$ as long as $I_{1}>(3.1)(2+2 \sqrt{5})^{3} \delta^{5}$. We need now to establish how large $I_{1}$ should be in order to replace $\xi_{\nu} / \xi_{\mu}$ or $\eta_{\nu} / \eta_{\mu}$ by $x_{\nu} / x_{\mu}$ or $y_{\nu} / y_{\mu}$ in the remaining steps of the algorithm.

Lemma 7.8. Let $\left(\xi_{\pi}, \eta_{\pi}\right)$ be the puncture of $\Pi=\left(p_{1}+p_{2} \delta+p_{3} \delta^{2}\right) / \sigma_{r} \in R_{r}$ such that $\xi_{\pi}>0, \xi_{\pi} \leqslant \xi_{\mu},\left|\eta_{\pi}\right| \leqslant\left|\eta_{\mu}\right|$, and let $\omega=\left(\xi_{\omega}, \eta_{\omega}\right)$ be the puncture of $\Omega$ $=\left(q_{1}+q_{2} \delta+q_{3} \delta^{2}\right) / \sigma_{r} \in R_{r}$. If $\left|\left[\xi_{\omega} / \xi_{\pi}\right]-\left[\eta_{\omega} / \eta_{\pi}\right]\right| \leqslant 2$ and $I_{1}>708 \delta^{5}$, then $\left[\xi_{\omega} / \xi_{\pi}\right]=\left[x_{\omega} / x_{\pi}\right]$ and $\left[\eta_{\omega} / \eta_{\pi}\right]=\left[y_{\omega} / y_{\pi}\right]$.

Proof. Let $k_{1}=\left[\xi_{\omega} / \xi_{\pi}\right], k_{2}=\left[\eta_{\omega} / \eta_{\pi}\right], \xi=\xi_{\omega}-k_{1} \xi_{\pi}, \eta=\eta_{\omega}-k_{1} \eta_{\pi}$, and $j=k_{1}-k_{2}$. We have $0<\xi<\xi_{\pi}$ and $-j<\eta / \eta_{\pi}<-j+1$. Now $\xi=$ $3 \delta(t+u \delta) / 2 \sigma_{r}$ and $\eta=\sqrt{3} \delta(t-u \delta) / 2 \sigma_{r}$, where $t=q_{2}-k_{1} p_{2}, u=q_{3}-k_{1} p_{3}$; consequently, $3 \delta t / \sigma_{r}=\xi+\sqrt{3} \eta, 3 \delta^{2} u / \sigma_{r}=\xi-\sqrt{3} \eta, 3 \delta\left(t-p_{2}\right) / \sigma_{r}=\xi-\xi_{\pi}+$ $\sqrt{3}\left(\eta-\eta_{\pi}\right)$, and $3 \delta^{2}\left(u-p_{3}\right) / \sigma_{r}=\xi-\xi_{\pi}-\sqrt{3}\left(\eta-\eta_{\pi}\right)$. Since $|j| \leqslant 2$, we have $|\eta|$, 
$\left|\eta-\eta_{\pi}\right|<3\left|\eta_{\pi}\right|$ and $|\xi|,\left|\xi-\xi_{\pi}\right|<\left|\xi_{\pi}\right|$; hence, $3 \delta^{2}|u| / \sigma_{r}$ and $3 \delta^{2}\left|u-p_{3}\right| / \sigma_{r}<$ $\left|\xi_{\pi}\right|+3 \sqrt{3}\left|\eta_{\pi}\right|<\left|\xi_{\mu}\right|+3 \sqrt{3}\left|\eta_{\mu}\right|<1+3 \sqrt{35} D / 2 \sigma_{r}$, by (6.14). Thus, we have $|u|,\left|u-p_{3}\right|<(1+\sqrt{35}) \delta$. Also,

$$
\begin{aligned}
9 \delta^{2} f(t, u) / \sigma_{r}^{2} & =(\xi+\sqrt{3} \eta)^{2}+|\xi+\sqrt{3} \eta| \xi-\sqrt{3} \eta \mid+(\xi-\sqrt{3} \eta)^{2} \\
& = \begin{cases}3 \xi^{2}+3 \eta^{2}<3 \xi_{\mu}^{2}+27 \eta_{\mu}^{2} & \left(\text { if } \xi^{2}>3 \eta^{2}\right) \\
\xi^{2}+9 \eta^{2}<\xi_{\mu}^{2}+81 \eta_{\mu}^{2} & \left(\text { if } \xi^{2}<3 \eta^{2}\right)\end{cases} \\
& <3 \cdot 307 D^{2} / \sigma_{r}^{2} ;
\end{aligned}
$$

and similarly,

$$
9 \delta^{2} f\left(t-p_{2}, u-p_{3}\right) / \sigma_{r}^{2}<3 \cdot 307 D^{2} / \sigma_{r}^{2}
$$

by (6.15). Also, since $0<\xi_{\pi}<\xi_{\mu}$ and $\left|\eta_{\pi}\right|<\left|\eta_{\mu}\right|$, one can show, as in Lemma 6.5 , that $\left|p_{3}\right|<(1+\sqrt{5}) \delta$; further, from (6.10) and (6.2) we get $f\left(p_{2}, p_{3}\right)<$ $\left(\left|p_{2}\right|+\delta\left|p_{3}\right|\right)^{2}<6\left|e_{r}\right| \delta<18 \delta^{4}$.

Since $I_{1}>708 \delta^{5}>(1+\sqrt{35}) \delta\left(307 \delta^{4}\right) / 3$, we have

$$
k_{1}=\left[\frac{q_{2}+q_{3} \delta}{p_{2}+p_{3} \delta}\right]=\left[\frac{x_{\omega}}{x_{\pi}}\right] \text { by Lemma 7.5. }
$$

By a somewhat similar argument, we can show that $k_{2}=\left[y_{\omega} / y_{\pi}\right]$. In this case we put $\xi=\xi_{\omega}-k_{2} \xi_{\pi}$ and $\eta=\eta_{\omega}-k_{2} \eta_{\pi}$. Then $j<\xi / \xi_{\pi}<j+1$ and $0<\eta / \eta_{\pi}<$ 1. We use (6.14) again with $c=\sqrt{3} / 3$ and the remaining parts of (6.15).

LEMma 7.9. Let $\pi$ and $\omega$ be defined as in Lemma 7.8 and suppose $\pi$ and $\omega$ form a basis of the lattice of punctures $L$ of $R_{r}$ If $I_{1}>(3.1)(3+\sqrt{5})^{3} \delta^{5} \simeq$ $445.02 \delta^{5}$ we must have $\left[x_{\omega} / x_{\pi}\right]=\left[\xi_{\omega} / \xi_{\pi}\right]$ when $\left|\eta_{\pi}\right|<1-\sqrt{3} / 4$ and $\left[y_{\omega} / y_{\pi}\right]=$ $\left[\eta_{\omega} / \eta_{\pi}\right]$ when $\left|\eta_{\pi}\right|>\sqrt{3} / 4$.

Proof. Since $\pi$ and $\omega$ form a basis of $L$, we must have $\left|p_{2} q_{3}-p_{3} q_{2}\right|=\left|e_{r}\right|$; also, as in Lemma 7.8, we have $\left|p_{3}\right|<(1+\sqrt{5}) \delta$. If $\left|\eta_{\pi}\right|>\sqrt{3} / 4$, then $\left|p_{2}-p_{3} \delta\right|$ $>\sigma_{r} / 2 \delta$; if $\left|\eta_{\pi}\right|<1-\sqrt{3} / 4<\sqrt{3} / 2$, then $\left|\xi_{\pi}\right|>\sqrt{\sqrt{3} / 2-3 / 16}>3 / 4$ (Lemma 4.2) and $\left|p_{2}+p_{3} \delta\right|>\sigma_{r} / 2 \delta$. Since $2\left|e_{r}\right| \delta / \sigma_{r} \leqslant 2 \delta$ and $I_{1}>3.1(3+\sqrt{5})^{3} \delta^{5}$, the result follows from Corollary 7.5.1.

Lemma 7.10. Let $\pi$ and $\omega$ be defined as in Lemma 7.8 and suppose $\pi$ and $\omega$ form a basis of L. If $\left|\left[x_{\omega} / x_{\pi}\right]-\left[y_{\omega} / y_{\pi}\right]\right| \leqslant 1$, then $\left[\xi_{\omega} / \xi_{\pi}\right]=\left[x_{\omega} / x_{\pi}\right]$ and $\left[\eta_{\omega} / \eta_{\pi}\right]=\left[y_{\omega} / y_{\pi}\right]$ when $I_{1}>708 \delta^{5}$.

Proof. We first note that

$$
\left|p_{2}+p_{3} \delta\right|\left|p_{2}-p_{3} \delta\right|=\left|\left(\left|p_{2}\right|+\left|p_{3} \delta\right|\right)\left(\left|p_{2}\right|-\left|p_{3} \delta\right|\right)\right| \geqslant \frac{1}{\left|p_{2}\right|+\left|p_{3} \delta\right|}
$$

by a direct application of Lemma 7.1. Also, since

$$
\left|\xi_{\pi}\right|<\left|\xi_{\mu}\right|, \quad\left|\eta_{\pi}\right|<\left|\eta_{\mu}\right|, \quad \text { and } \frac{2 \sigma_{r}}{3 \delta}\left|\xi_{\mu}\right|, \quad \frac{2 \sigma_{r}}{\sqrt{3} \delta}\left|\eta_{\mu}\right|<\sqrt{6\left|e_{r}\right| \delta}
$$


(by (6.10)), we see that $\left|p_{2}\right|+\left|p_{3}\right| \delta<\sqrt{6\left|e_{r}\right| \delta}$. Hence,

$$
\left|p_{2}^{2}-p_{3}^{2} \delta\right|>\frac{1}{\sqrt{6\left|e_{r}\right| \delta}} \text {. }
$$

Since $3 D>\left|e_{r}\right|, 3 \sqrt{18} \delta^{5}>\left|e_{r}\right| \sqrt{6\left|e_{r}\right| \delta}$, and $\left|p_{3}\right|<(1+\sqrt{5}) \delta$, we get

$$
2\left(2 p_{3}^{2} \delta+\left|e_{r}\right|\right) \sqrt{6\left|e_{r}\right| \delta}<2\left(2(1+\sqrt{5})^{2}+3\right) \sqrt{18} \delta^{5}<I_{1} .
$$

Put $\gamma=\left(\delta I_{1}-\left[\delta I_{1}\right]\right) / I_{1}$. Then $0<\gamma<I_{1}^{-1}$ and

$$
2 \gamma\left(2 p_{3}^{2}+\left|e_{r}\right|\right)<\frac{I_{1} \gamma}{\sqrt{6\left|e_{r}\right| \delta}}<\frac{1}{\sqrt{6\left|e_{r}\right| \delta}} .
$$

If we put $u=\left(I_{1}^{2} p_{2}^{2}-p_{3}^{2}\left[I_{1} \delta\right]^{2}\right) / I_{1}^{2}, v=p_{2}^{2}-\delta^{2} p_{3}^{2}$, and $d=u-v$, then $0<$ $d<2 \delta \gamma p_{3}^{2}$. Also, since

$$
\left|\left[\frac{x_{\omega}}{x_{\pi}}\right]-\left[\frac{y_{\omega}}{y_{\pi}}\right]\right| \leqslant 1
$$

we have

$$
\left|\frac{x_{\omega}}{x_{\pi}}-\frac{y_{\omega}}{y_{\eta}}\right| \leqslant 2
$$

From (7.4), it follows that $|u| \geqslant\left[I_{1} \delta\right]\left|e_{r}\right| / I_{1}=(\delta-\gamma)\left|e_{r}\right|$. Since $|u| /|v| \leqslant 1+$ $d /|v|$, we get

$$
\frac{(\delta-\gamma)\left|e_{r}\right|}{\left|p_{2}^{2}-p_{3}^{2} \delta^{2}\right|} \leqslant 1+\frac{d}{\left|p_{2}^{2}-p_{3}^{2} \delta^{2}\right|}
$$

Adding $\gamma\left|e_{r}\right| /\left|p_{2}^{2}-p_{3}^{2} \delta^{2}\right|$ to both sides of (7.7) and multiplying through by 2, we have

$$
\frac{2 \delta\left|e_{r}\right|}{\left|p_{2}^{2}-p_{3}^{2} \delta^{2}\right|} \leqslant 2+2 \frac{d+\gamma\left|e_{r}\right|}{\left|p_{2}^{2}-p_{3}^{2} \delta^{2}\right|}<2+\frac{d+\gamma\left|e_{r}\right|}{\gamma\left(2 \delta p_{3}^{2}+\left|e_{r}\right|\right)}<3,
$$

where the last inequalities follow from (7.5). (7.6) and the fact that $0<d<2 p_{3}^{2} \gamma \delta$.

Since $\left|p_{2}\right|+\left|p_{3}\right| \delta<\sqrt{6\left|e_{r}\right| \delta}$, we see from (7.8) and (7.5) that

$$
\left|p_{2}-p_{3} \delta\right|,\left|p_{2}+p_{3} \delta\right| \geqslant|| p_{2}|-| p_{3}|\delta|>2 \sqrt{\delta\left|e_{r}\right|} / 3 \sqrt{6} .
$$

Now

$$
\left|x_{\pi}\right|=\left|I_{1}\left(p_{2}+p_{3} \delta\right)-I_{1} \gamma p_{3}\right|>2 I_{1} \sqrt{\delta\left|e_{r}\right|} / 3 \sqrt{6}-(1+\sqrt{5}) \delta ;
$$

hence, $\left|x_{\pi}\right|\left|p_{2}+p_{3} \delta\right|>\left|e_{r}\right|$ and from (7.2), we see that $\left|\xi_{\omega}\right| \xi_{\pi}-x_{\omega} / x_{\pi} \mid<1$. Similarly, we are able to show that $\left|\eta_{\omega} / \eta_{\pi}-y_{\omega} / y_{\pi}\right|<1$. By Lemma 7.9 either $\left[\xi_{\omega} / \xi_{\pi}\right]$ $=\left[x_{\omega} / x_{\pi}\right]$ or $\left[\eta_{\omega} / \eta_{\pi}\right]=\left[y_{\omega} / y_{\pi}\right]$; consequently, $\left|\left[\xi_{\omega} / \xi_{\pi}\right]-\left[\eta_{\omega} / \eta_{\pi}\right]\right| \leqslant 2$ and our result follows from Lemma 7.8 .

LeMma 7.11. Let $\pi$ and $\omega$ be defined as in Lemma 7.8 and suppose that $\pi$ and $\omega$ form a basis of $L$. If $j=\left[x_{\omega} / x_{\pi}\right]-\left[y_{\omega} / y_{\pi}\right]\left(I_{1}>708 \delta^{5}\right)$, then $j$ and $\left[\xi_{\omega} / \xi_{\pi}\right]-$ $\left[\eta_{\omega} / \eta_{\pi}\right]$ have the same sign. 
Proof. By Lemma 7.10 the result is certainly true when $j \leqslant 1$ and by Lemma 7.8 the result is true when $\left|\left[\xi_{\omega} / \xi_{\pi}\right]-\left[\eta_{\omega} / \eta_{\pi}\right]\right| \leqslant 2$. Assume that $j>1$ and $\left|\left[\xi_{\omega} / \xi_{\pi}\right]-\left[\eta_{\omega} / \eta_{\pi}\right]\right|>2$. The sign of $\left[\xi_{\omega} / \xi_{\pi}\right]-\left[\eta_{\omega} / \eta_{\pi}\right]$ is the same as that of $\xi_{\omega} / \xi_{\pi}-\eta_{\omega} / \eta_{\pi}=3 \sqrt{3} \delta^{3} e_{r} / 2 \xi_{\pi} \eta_{\pi}$ and the sign of $\left[x_{\omega} / x_{\pi}\right]-\left[y_{\omega} / y_{\pi}\right]$ is the same as that of $I_{1}\left[I_{1} \delta\right] e_{r} / x_{\pi} y_{\pi}$. Since $x_{\pi} \xi_{\pi}$ and $\eta_{\pi} y_{\pi}>0$, the result follows.

We are finally able to present the main result of this section. We give here

Algorithm $\mathrm{B}_{1}$. Let $I_{1}, I_{2}$ be any integers such that $I_{1}>841 \delta^{5}, I_{2}>80 \delta^{2}$. Also, let $[1, M, N]$ be the basis $\left[1,1 / \theta_{g}^{(r-1)}, \theta_{h}^{(r-1)} / \theta_{g}^{(r-1)}\right]$ of $R_{r}$; and let $M=$ $\left(m_{1}+m_{2} \delta+m_{3} \delta^{2}\right) / \sigma_{r}$ and $N=\left(n_{1}+n_{2} \delta+n_{3} \delta^{2}\right) / \sigma_{r}$. Perform the following steps in the indicated order:

(i) Transform the basis $[1, M, N]$ of $R_{r}$ by $K_{2}(-k, 1)$, where $k=\left[n_{3} / m_{3}\right]$ when $m_{3} \neq 0$ and $k=\left[n_{2} / m_{2}\right]$ otherwise.

(ii) Transform the new basis by $\left(\begin{array}{cc}k_{1} & 0 \\ 0 & k_{2}\end{array}\right)$, where $k_{1}=\operatorname{sgn}\left(x_{\mu}\right), k_{2}=\operatorname{sgn}\left(x_{\nu}\right)$.

(iii) If $x_{\nu}>x_{\mu}$ go to (iv); otherwise, transform the basis by $K_{2}(1,1)$ and go to (iv) unless $y_{\nu} y_{\mu}<0$ and $\left|y_{\nu}\right|>\left|y_{\mu}\right|$. If this latter case occurs, transform the basis by $K_{1}(0,1)$ instead of $K_{2}(1,1)$ and terminate $\mathrm{B}_{1}$.

(iv) If $y_{\mu} y_{\nu}<0$, go to step (v); otherwise

(1) If $\left[y_{\nu} / y_{\mu}\right]=\left[x_{\nu} / x_{\mu}\right]=k$, transform the basis by $K_{1}(-k, 1)$ until $\left[y_{\nu} / y_{\mu}\right] \neq\left[x_{\nu} / x_{\mu}\right]$. When such a basis is found execute one of the following steps.

(2) If $\left[x_{\nu} / x_{\mu}\right]+1=\left[y_{\nu} / y_{\mu}\right]=k$, transform the basis by $K_{1}(k,-1)$ and go to $(\mathrm{v})$.

(3) If $k=\left[x_{\nu} / x_{\mu}\right]=\left[y_{\nu} / y_{\mu}\right]+1$, transform the basis by $K_{1}(-k, 1)$ and go to $(\mathrm{v})$.

(4) If $\left[x_{\nu} / x_{\mu}\right]<\left[y_{\nu} / y_{\mu}\right]-1$, transform the basis by $K_{1}\left(\left[x_{\nu} / x_{\mu}\right]+1,-1\right)$ when $\left|\bar{y}_{\mu}\right|<I_{2} \sigma_{r}$; otherwise, transform the basis by $K_{2}\left(\left[y_{\nu} / y_{\mu}\right],-1\right)$. Terminate $\mathbf{B}_{1}$.

(5) If $\left[x_{v} / x_{\mu}\right]>\left[y_{\nu} / y_{\mu}\right]+1$, transform the basis by $K_{1}\left(-\left[x_{v} / x_{\mu}\right], 1\right)$ when $\left|\bar{y}_{\mu}\right|<I_{2} \sigma_{r}$; otherwise, transform the basis by $K_{2}\left(-\left[y_{v} / y_{\mu}\right]-1,1\right)$. Terminate $B_{1}$.

(v) If $\left|y_{\mu}\right|>\left|y_{\nu}\right|$ terminate $\mathrm{B}_{1}$. If $\left|y_{\mu}\right| \leqslant\left|y_{\nu}\right|$ transform the basis by $K_{1}\left(-\left[x_{v} / x_{\mu}\right], 1\right)$ when $\left|\bar{y}_{\mu}\right|<I_{2} \sigma_{r}$; otherwise, transform by $K_{2}\left(\left[-y_{v} / y_{\mu}\right], 1\right)$.

THEOREM 7.12. If $[1, \Lambda, P]$ is the basis of $R_{r}$ which results on executing Algorithm $\mathrm{B}_{1}$, then $\xi_{\rho}>\xi_{\lambda}>0$ and $\left|\eta_{\rho}\right|<\left|\eta_{\lambda}\right|, \eta_{\lambda} \eta_{\rho}<0$.

Proof. If, starting with the basis $[1, \bar{M}, \bar{N}]$ which results after performing step (i) of Algorithm $B_{1}$, we execute steps (i)-(iv) of Algorithm A, we will certainly produce a basis of $R$ with the same properties as $[1, \Lambda, P]$ above. We have already seen in Lemma 7.7 that, in this case, steps (i) and (ii) of A can be replaced by steps (ii) and (iii) of $B_{1}$.

If $\bar{\mu}=\left(\bar{\xi}_{\mu}, \bar{\eta}_{\mu}\right), \bar{v}=\left(\bar{\xi}_{v}, \bar{\eta}_{v}\right)$ are the punctures of $\bar{M}$ and $\bar{N}$ and $\mu=\left(\xi_{\mu}, \eta_{\mu}\right)$, $v=\left(\xi_{v}, \eta_{v}\right)$ are the punctures of the basis produced by Algorithm A at the beginning 
of step (iii), then $\left|\xi_{\mu}\right|=\left|\bar{\xi}_{\mu}\right|=3 \delta\left(\bar{m}_{2}+\delta \bar{m}_{3}\right) / 2 \sigma_{r},\left|\eta_{\mu}\right|=\left|\bar{\eta}_{\mu}\right|$ and $\left|n_{3}\right| \leqslant 2\left|\bar{m}_{3}\right|<$ $2(1+\sqrt{5}) \delta$. Indeed, if $\eta_{v} \eta_{\mu}<0$, then $\left|\eta_{v}\right|=\left|\bar{\eta}_{v}\right|$. Thus, by Lemma 7.4, $\operatorname{sgn}\left(\eta_{\mu} \eta_{v}\right)=$ $\operatorname{sgn}\left(y_{\mu} y_{v}\right)$. Now at any point in the execution of substep (a) of A(iii), our new punctures $\mu$ and $v$ satisfy the inequalities $\left|\xi_{\mu}\right| \leqslant\left|\bar{\xi}_{\mu}\right|$ and $\left|\eta_{\mu}\right| \leqslant\left|\bar{\eta}_{\mu}\right|$; thus, if $\mid\left[x_{v} / x_{\mu}\right]-$ $\left[y_{v} / y_{\mu}\right] \mid \leqslant 1$, then $\left[x_{v} / x_{\mu}\right]=\left[\xi_{v} / \xi_{\mu}\right],\left[y_{v} / y_{\mu}\right]=\left[\eta_{v} / \eta_{\mu}\right]$ by Lemma 7.10. Also, if $\left[x_{v} / x_{\mu}\right]>\left[y_{v} / y_{\mu}\right]+1$, then $\left[\xi_{v} / \xi_{\mu}\right]>\left[\eta_{v} / \eta_{\mu}\right]+1$; and if $\left[y_{v} / y_{\mu}\right]>\left[x_{v} / x_{\mu}\right]+1$, then $\left[\eta_{v} / \eta_{\mu}\right]>\left[\xi_{v} / \xi_{\mu}\right]+1$ by Lemmas 7.10 and 7.11. Now, if $\left|\bar{y}_{\mu}\right|<I_{2} \sigma_{r}$, then $\left|\eta_{\mu}\right|<$ $1-\sqrt{3} / 4$ and if $\left|\bar{y}_{\mu}\right| \geqslant I_{2} \sigma_{r}$, then $\left|\eta_{\mu}\right|>\sqrt{3} / 4$ by Lemma 7.6 ; hence, by Lemma 7.9, if $\left|\bar{y}_{\mu}\right|<I_{2} \sigma_{r}$, then $\left[x_{v} / x_{\mu}\right]=\left[\xi_{v} / \xi_{\mu}\right]$ and if $\bar{y}_{\mu} \mid \geqslant I_{2} \sigma_{r}$, then $\left[y_{v} / y_{\mu}\right]=\left[\eta_{v} / \eta_{\mu}\right]$. It follows that we can replace step (iii) of $A$ by step (iv) of $B_{1}$ and still obtain a result that some form of Algorithm A would produce.

If we use Algorithm $\mathrm{A}$ on $[1, \bar{M}, \bar{N}]$ and arrive at the beginning of step (iv) with a new basis with punctures $\mu=\left(\xi_{\mu}, \eta_{\mu}\right), v=\left(\xi_{v}, \eta_{v}\right)$, then either $0<\xi_{\mu}=\left|\bar{\xi}_{\mu}\right|,\left|\eta_{\mu}\right|=$ $\left|\bar{\eta}_{\mu}\right|,\left|\eta_{v}\right|=\left|\bar{\eta}_{v}\right|$ or $0<\xi_{\mu}<\xi_{v} \leqslant\left|\xi_{\mu}\right|,\left|\eta_{\mu}\right| \leqslant\left|\bar{\eta}_{\mu}\right|,\left|\eta_{v}\right| \leqslant\left|\bar{\eta}_{\mu}\right|$, the latter case occurring when either (iii)-b or (iii)-c is executed. It follows that in each of these cases we have $\left|n_{3}\right|,\left|m_{3}\right|<(1+\sqrt{5}) \delta$. By Lemmas 7.4, 7.6, and 7.9 we see that step (v) of $B_{1}$ can be used to replace step (iv) of $A$ and the basis $[1, \Lambda, P]$ which results on performing this step will have the required properties.

In the next section we show how we can modify step (v) of Algorithm A in order that only integers are used.

8. Modification of Step (v) of Algorithm A. Let $\mu=\left(\xi_{\mu}, \eta_{\mu}\right), \nu=\left(\xi_{\nu}, \eta_{\nu}\right)$ be the punctures of $M=\left(m_{1}+m_{2} \delta+m_{3} \delta^{2}\right) / \sigma_{r}, N=\left(n_{1}+n_{2} \delta+n_{3} \delta^{2}\right) / \sigma_{r}$, respectively. For the result that follows we assume that $\mu, \nu$ forms the basis of $L$ which results on applying Algorithm $\mathbf{B}_{1}$. For this basis we have

$$
\xi_{\nu}>\xi_{\mu}>0, \quad\left|\eta_{\mu}\right|>\left|\eta_{\nu}\right|, \quad \eta_{\mu} \eta_{\nu}<0 .
$$

We must now prove some preliminary lemmas.

LEMMA 8.1. For the $\mu$ and $\nu$ described above, we have

$$
\xi_{\mu} / 3, \xi_{\nu} / 3,\left|\eta_{\mu}\right| / \sqrt{3},\left|\eta_{\nu}\right| / \sqrt{3}<(3+2 \sqrt{5}) D / 2 \sigma_{r} \text {. }
$$

Proof. We first note that since $\eta_{\nu}$ and $\eta_{\mu}$ have different signs and $\xi_{\nu}, \xi_{\mu}>0$, we have $\xi_{\mu}\left|\eta_{\nu}\right|, \xi_{\nu}\left|\eta_{\mu}\right|<E_{r}$. Thus, if $\left|\eta_{\nu}\right|<1-\sqrt{3} / 4<\sqrt{3} / 2$, then $\xi_{\nu}>$ $\sqrt{\sqrt{3} / 2-3 / 16}>3 / 4$ (Lemma 4.2) and $\left|\eta_{\mu}\right| / \sqrt{3}<4 E_{r} / 3 \sqrt{3}=2 D\left|e_{r}\right| / \sigma_{r}^{2}<2 D / \sigma_{r}$ by (6.2). Also, if $\eta_{\mu}>\sqrt{3} / 4$, then $\xi_{\nu} / 3<2 D / \sigma_{r}$.

If we arrive at the end of $B_{1}$ by skipping over all the substeps of (iv) and step (v), then, since $\left|\xi_{\mu}\right|,\left|\eta_{\mu}\right|$ do not change after (i) has been executed, we get

$$
\begin{gathered}
\xi_{\mu} / 3<\delta \sqrt{6\left|e_{r}\right| \delta} / 2 \sigma_{r}<3 \sqrt{2} D / 2 \sigma_{r}, \\
\left|\eta_{\nu}\right| / \sqrt{3}<\left|\eta_{\mu}\right| / \sqrt{3}<\delta \sqrt{5\left|e_{r}\right| \delta / 2 \sigma_{r}}<\sqrt{15} D / 2 \sigma_{r}
\end{gathered}
$$

by (6.10). We also have either $\xi_{\nu} / 3<2 \xi_{\mu} / 3<6 D / 2 \sigma_{r}$ or $\left|n_{3}\right|<(1+\sqrt{5}) \delta$. In the latter case, if $\left|\eta_{\nu}\right|<1 / 2 \sqrt{3}$, then $\left|n_{2}-n_{3} \delta\right|<\sigma_{r} / 3 \delta<\delta^{2}$ and $2 \sigma_{r} \xi_{\nu} / 3 \delta<\delta^{2}+$ $2(1+\sqrt{5}) \delta^{2}=(3+2 \sqrt{5}) \delta^{2}$. If $\left|\eta_{\nu}\right|>1 / 2 \sqrt{3}$, then $\left|\eta_{\mu}\right|>1 / 2 \sqrt{3}$ and $\xi_{\nu} / 3<$ $2 \sqrt{3} E_{r} / 3<6 D / 2 \sigma_{r}$. 
If we arrive at the end of $B_{1}$ by executing step (iv) or step (v), then we must execute one of substep (4) of (iv) or substep (5) of (iv) or step (v). If $\bar{\mu}=\left(\bar{\xi}_{\mu}, \bar{\eta}_{\mu}\right)$ is the puncture of $M$ just before either of (iv) 4 , (iv)-5 or (v) is executed, we get

$$
\left|\bar{\xi}_{\mu}\right|,\left|\bar{\eta}_{\mu}\right|<\frac{3 \delta}{2 \sigma_{r}} \sqrt{6\left|e_{r}\right| \delta}<\frac{9 \sqrt{2} D}{2 \sigma_{r}}
$$

by using the reasoning in Theorem $7.12,(6.10)$ and (6.2). If, in any of these steps, we start with $\left|\bar{y}_{\bar{\mu}}\right|<I_{2} \sigma_{r}$, then, since a transformation of the form $K_{1}(a, b)$ is used to obtain $\mu$ and $\nu$, we get $\nu=\bar{\mu}$. Hence, by Lemma 7.6 , we see that $\left|\eta_{\nu}\right|<1-\sqrt{3} / 4$ and, therefore,

$$
\left|\eta_{\nu}\right| / \sqrt{3}<(1-\sqrt{3} / 4) / \sqrt{3}<3 D / \sigma_{r}, \quad\left|\eta_{\mu}\right| / \sqrt{3}<2 D / \sigma_{r}, \quad \xi_{\mu} / 3<\xi_{\nu} / 3<3 \sqrt{2} D / 2 \sigma_{r} .
$$

If we start with $\left|\bar{y}_{\bar{\mu}}\right| \geqslant I_{2} \sigma_{r}$, then a transformation of the form $K_{2}(a, b)$ is used and $\mu=\bar{\mu}$. It follows in this case that $\left|\eta_{\mu}\right|>\sqrt{3} / 4$ and

$$
\xi_{\mu} / 3<\xi_{\nu} / 3<2 D / \sigma_{r}, \quad\left|\eta_{\nu}\right| / \sqrt{3}<\left|\eta_{\mu}\right| / \sqrt{3}<3 \sqrt{6} D / 2 \sigma_{r} .
$$

The lemma is now proved.

LEMmA 8.2. Let $\mu, v$ be any basis of $L$ such that (8.1) is true. If $\left|\eta_{\mu}\right|>\sqrt{3} / 4$, $\left|\bar{y}_{v}\right|>I_{2} \sigma_{r}\left(I_{2}>80 \delta^{2}\right),\left|\eta_{v}\right| / \sqrt{3}<(3+2 \sqrt{5}) D / 2 \sigma_{r}$, then $\left|\eta_{v}\right|>\sqrt{3} / 4,\left|n_{2}\right|+\left|n_{3}\right| \delta<$ $(3+2 \sqrt{5}) \delta^{2}$, and $\left|n_{2}\right|,\left|\delta n_{3}\right|<(2+\sqrt{5}) \delta^{2} ;$ if $\left|\eta_{\nu}\right|<1-\sqrt{3} / 4,\left|\bar{y}_{\mu}\right| \leqslant I_{2} \sigma_{r}, \xi_{\mu} / 3<$ $(3+2 \sqrt{5}) D / 2 \sigma_{r}$, then $\left|\eta_{\mu}\right|<1-\sqrt{3} / 4,\left|m_{2}\right|+\left|m_{3}\right| \delta<(3+2 \sqrt{5}) \delta^{2}$, and $\left|m_{2}\right|$, $\left|\delta m_{3}\right|<(2+\sqrt{5}) \delta^{2}$.

Proof. We prove the second part of this lemma here. The proof of the first part is similar and somewhat easier.

Since $\left|\eta_{\nu}\right|<1-\sqrt{3} / 4<\sqrt{3} / 2$ and $\left|\eta_{\mu}\right| \xi_{\nu}<E_{r}$, we have $\xi_{\nu}>\sqrt{1-\eta_{\nu}^{2}}>3 / 4$ (Lemma 4.2) and $\left|\eta_{\mu}\right|<4 E_{r} / 3$. It follows that $\left|m_{2}-m_{3} \delta\right|<4\left|e_{r}\right| \delta^{2} / \sigma_{r} \leqslant 4 \delta^{2}$. Since $\left|m_{2}+m_{3} \delta\right|<(3+2 \sqrt{5}) \delta^{2},\left|m_{2}\right|+\left|m_{3} \delta\right|<\max \left\{(3+2 \sqrt{5}) \delta^{2}, 4 \delta^{2}\right\}=$ $(3+2 \sqrt{5}) \delta^{2}$ and from Lemma 7.6 we see that $\left|\eta_{\mu}\right|<1-\sqrt{3} / 4$. We also note that since $\left|\eta_{\mu}\right| \xi_{\mu}<\xi_{\nu}\left|\eta_{\mu}\right|<E_{r}$, we have

$$
\left|m_{2}^{2}-m_{3}^{2} \delta^{2}\right|<2\left|e_{r}\right| \delta<6 \delta^{4} .
$$

If $\left|m_{2}-m_{3} \delta\right|<\delta^{2}$, then $\left|m_{2}\right|,\left|\delta m_{3}\right|<\left(\delta^{2}+(3+2 \sqrt{5}) \delta^{2}\right) / 2<$ $(2+\sqrt{5}) \delta^{2}$. If $\delta^{2}<\left|m_{2}-m_{3} \delta\right|<2 \delta^{2}$, then, from (8.2), we have $\left|m_{2}+\delta m_{3}\right|<6 \delta^{2}$ and $\left|m_{2}\right|,\left|\delta m_{3}\right|<\left(2 \delta^{2}+6 \delta^{2}\right) / 2=4 \delta^{2}$. Finally if $\left|m_{2}-m_{3} \delta^{2}\right|>2 \delta^{2}$, then $\left|m_{2}+\delta m_{3}\right|<3 \delta^{2}$ and $\left|m_{2}\right|,\left|\delta m_{3}\right|<3.5 \delta^{2}$.

We now consider the following algorithm (Algorithm $\mathrm{B}_{2}$ ). We assume that we begin with a basis $[1, M, N]$ whose punctures $\mu=\left(\xi_{\mu}, \eta_{\mu}\right), \nu=\left(\xi_{\nu}, \eta_{\nu}\right)$ have the properties (8.1).

Algorithm $\mathrm{B}_{2}$. If $\left|\bar{y}_{\mu}\right| \geqslant I_{2} \sigma_{r}$ and $\left|\bar{y}_{\nu}\right| \leqslant I_{2} \sigma_{r}$, we terminate the algorithm immediately. When these conditions are not satisfied and $\left|\bar{y}_{\mu}\right|<I_{2} \sigma_{r}$, execute step (1); otherwise, execute step (2). 
(1) Transform the basis $[1, M, N]$ by $K_{1}(-k, 1)$, where $k=\left[x_{\nu} / x_{\mu}\right]$. Continue doing this until $\left|\bar{y}_{\mu}\right| \geqslant I_{2} \sigma_{r}$. At this point terminate the algorithm.

(2) Transform the basis $[1, M, N]$ by $K_{3}(1, k)$, where $k=\left[-y_{\mu} / y_{\nu}\right]$. Continue doing this until $\left|\bar{y}_{\nu}\right| \leqslant I_{2} \sigma_{r}$. At this point terminate the algorithm.

Put $\left[1, M_{r}^{*}, N_{r}^{*}\right]=\left[1,1 / \theta_{g}^{(r-1)}, \theta_{h}^{(r-1)} / \theta_{g}^{(r-1)}\right]$, where $\theta_{g}^{(r-1)}$ and $\theta_{h}^{(r-1)}$ are defined in Section 2. We now prove

THEOREM 8.3. Let $I_{1}>3.1(2+\sqrt{5})^{3} \delta^{5}$ and $I_{2}>80 \delta^{2}$. If the basis $[1, M, N]$ is the basis of $R_{r}$ which results on applying the steps of the Algorithm $\mathrm{B}_{1}$ to $\left[1, M_{r}^{*}, N_{r}^{*}\right]$, then Algorithm $\mathrm{B}_{2}$ terminates.

Proof. Suppose we deal with step (2) of $\mathrm{B}_{2}$. By Lemma 8.1 we have $\left|n_{2}\right|+$ $\delta\left|n_{3}\right|<(3+2 \sqrt{5}) \delta^{2}$; and since $\left|\bar{y}_{\nu}\right|>I_{2} \sigma_{r}$, we have $\left|\eta_{\nu}\right|>\sqrt{3} / 4$ by Lemma 7.6. From (8.1) it follows that $\left|\eta_{\mu}\right|>\sqrt{3} / 4$ and from Lemma 8.2, we get $\left|n_{3}\right|<$ $(2+\sqrt{5}) \delta$. Thus, since

$$
\left|e_{r}\right| /\left|n_{2}-\delta n_{3}\right|+\left|n_{3}\right|<2 \delta\left|e_{r}\right| / \sigma_{r}+(2+\sqrt{5}) \delta<(2+2 \sqrt{5}) \delta,
$$

we deduce from Corollary 7.5.1 that $k=\left[\left|\eta_{\mu} / \eta_{\nu}\right|\right]=\left[\left|y_{\mu} / y_{\nu}\right|\right]$. If we put $\eta_{\nu}^{(1)}=$ $\eta_{\mu}, \eta_{\nu}^{(2)}=\eta_{\nu}, k_{i}=\left[\left|y_{\nu}^{(i)} / y_{\nu}^{(i+1)}\right|\right]$, we see by Lemma 8.2 that step (2) of $\mathrm{B}_{2}$ will generate a sequence

$$
\eta_{\nu}^{(1)}, \eta_{\nu}^{(2)}, \eta_{\nu}^{(3)}, \ldots, \eta_{\nu}^{(h)}, \ldots
$$

where $\left|\bar{y}_{\nu}^{(j+1)}\right|>I_{2} \sigma_{r}, \eta_{\nu}^{(j+2)}=k_{j} \eta_{\nu}^{(j+1)}+\eta_{\nu}^{(j)}, \eta_{\nu}^{(j+2)} \eta_{\nu}^{(j+1)}<0$ and

$$
\left|\eta_{\nu}^{(1)}\right|>\left|\eta_{\nu}^{(2)}\right|>\left|\eta_{\nu}^{(3)}\right|>\cdots>\left|\eta_{\nu}^{(h)}\right|>\cdots
$$

By Lemma 8.2, we know that, if $2 \sigma_{r} \eta_{\nu}^{(j)} / \sqrt{3} \delta=n_{2}^{(j)}+n_{3}^{(j)} \delta$, then the values of $\left|n_{2}^{(j)}\right|$ and $\left|\delta n_{3}^{(j)}\right|$ are bounded by $(2+\sqrt{5}) \delta^{2}$, thus there can be at most $4(2+\sqrt{5})^{2} D$ different pairs $\left(n_{2}^{(j)}, n_{3}^{(j)}\right)$. If $\left|\bar{y}_{\nu}^{(j)}\right|>I_{2} \sigma_{r}$ for $j=2,3,4, \ldots, h>$ $4(2+\sqrt{5})^{2} D$, we must have $r<s<h$ such that $\left(n_{2}^{(r)}, n_{3}^{(r)}\right)=\left(n_{2}^{(s)}, n_{3}^{(s)}\right)$. Since $\left|\eta_{\nu}^{(r)}\right|>\left|\eta_{\nu}^{(s)}\right|$, this is impossible. It follows that we must eventually find some $h \leqslant$ $4(2+\sqrt{5}) D^{2}$ such that $\left|\bar{y}_{\nu}^{(h)}\right| \leqslant I_{2} \sigma_{r}$. The proof that step (1) of $\mathrm{B}_{2}$ terminates when $\left|\bar{y}_{\mu}\right|<I_{2} \sigma_{r}$ is similar to the above.

When, after being applied to the basis $[1, M, N]$ above, Algorithm $\mathrm{B}_{2}$ terminates we have a new basis $[1, \Psi, \Phi]$ of $R_{r}$, where

$$
\Phi=\left(s_{1}+s_{2} \delta+s_{3} \delta^{2}\right) / \sigma_{r}, \quad \Psi=\left(t_{1}+t_{2} \delta+t_{3} \delta^{2}\right) / \sigma_{r} .
$$

If $\phi=\left(\xi_{\phi}, \eta_{\phi}\right)$ is the puncture of $\Phi$ and $\psi=\left(\xi_{\psi}, \eta_{\psi}\right)$ is the puncture of $\Psi$, then

$$
\left|\eta_{\phi}\right|<\left|\eta_{\psi}\right|, \quad \eta_{\phi} \eta_{\psi}<0, \quad \xi_{\phi}>\xi_{\psi}>0, \quad\left|\bar{y}_{\phi}\right| \leqslant \sigma_{r} I_{2} \quad \text { and } \quad\left|\bar{y}_{\psi}\right| \geqslant \sigma_{r} I_{2} \text {. }
$$

LEMMA 8.4. If $I_{2}>128 \sqrt{3} \delta^{5} / 3 \simeq 73.90 \delta^{5}$, then $[1, \Phi, \Psi]$ is a $\beta$-basis.

Proof. If we find $\Phi$ and $\Psi$ by executing the steps of part (2) of $\mathrm{B}_{2}$, we have $2 \sigma_{r}\left|\eta_{\psi}\right| / \sqrt{3} \delta, 2 \sigma_{r}\left|\eta_{\phi}\right| / \sqrt{3} \delta<(3+2 \sqrt{5}) \delta^{2}$. Also, since $\eta_{\psi}$ precedes $\eta_{\phi}$ in (8.3) we have $\left|\eta_{\psi}\right|>\sqrt{3} / 4$ by Lemma 7.6. It follows that $\left|s_{2}+\delta s_{3}\right|<4 \delta^{2}$ and $\left|s_{2}-\delta s_{3}\right|$ $<(3+2 \sqrt{5}) \delta^{2}$ and by Lemma 7.6, $\left|\eta_{\phi}\right|<1-\sqrt{3} / 4$. By similar reasoning we can 
show that if we execute the steps of part (1) of $\mathrm{B}_{2}$, we have $\left|\eta_{\phi}\right|<1-\sqrt{3} / 4,\left|\eta_{\psi}\right|$ $>\sqrt{3} / 4$. If $\Phi$ and $\Psi$ are found without having to execute either step (1) or (2) of $\mathrm{B}_{2}$, then $\left|\eta_{\phi}\right|<1-\sqrt{3} / 4$ and $\left|\eta_{\psi}\right|>\sqrt{3} / 4$ by Lemmas 7.6 and 8.1 .

Now $\left|\eta_{\psi}\right|=\left|\bar{y}_{\psi}\right| / 2 \sigma_{\gamma_{2}}+\gamma \geqslant 1 / 2-|\gamma|$, where $|\gamma|<\left(\left|t_{2}\right|+\left|t_{3}\right| \delta\right) / 2 \sigma_{r} I_{2}<$ $2 \delta^{2} / \sigma_{r} I_{2}$, from the proof of Lemma 6.1. Also,

$$
\left|\eta_{\phi}\right| / 2=\sqrt{3} \delta\left|s_{2}-s_{3} \delta\right| / 4 \sigma_{r}>\sqrt{3} \delta /\left(4 \sigma_{r}\left(\left|s_{2}\right|+\left|s_{3}\right| \delta\right)^{2}\right) \quad(\text { Lemma } 7.1)
$$

hence, from Lemma 6.1,

$$
\left|\eta_{\phi}\right| / 2>\frac{\sqrt{3}}{64 \sigma_{r} D}>\frac{2 \delta^{2}}{\sigma_{r} I_{2}}>|\gamma|,
$$

and $\left|\eta_{\psi}\right|>\left(1-\left|\eta_{\phi}\right|\right) / 2$.

Let Algorithm $\mathrm{B}$ be that algorithm which we obtain on using Algorithm $\mathrm{B}_{1}$ to substitute for the first four steps ((i), (ii), (iii), (iv)) of Algorithm A and Algorithm $B_{2}$ for the fifth step (v) of A with $I_{1}>3.1(2+2 \sqrt{5})^{3} \delta^{5}$ and $I_{2}>128 \sqrt{3} \delta^{5} / 3$. We have now proved the following

THEOREM 8.5. If $\theta$ is the puncture of $\theta_{g}^{(r)}$, the relative minimum of the second kind adjacent to 1 in $R_{r}$, then

$$
\theta=a \phi+b \psi
$$

where $(a, b) \in\{(1,0),(0,1),(1,-1),(1,1),(2,1)\}$ and $\phi$ and $\psi$ are the punctures of $\Phi$ and $\Psi$ found by using Algorithm B on the basis $\left[1, M_{r}^{*}, N_{r}^{*}\right]$ of $R_{r}$.

In the next section we show how to find $\theta_{g}^{(r)}$ once $\Phi$ and $\Psi$ are known. As in Algorithm B, we wish to use as much integer arithmetic as possible.

9. Determination of $\theta_{g}^{(r)}$. Let $[1, \Phi, \Psi]$ in (8.4) be a $\beta$-basis. In order to determine the set $S$ in Section 5 we need to be able to discover when $\xi_{\psi}>1 / 3, \xi_{\psi}<$ $1 / 2$, and $\left|\eta_{\psi}\right|<\sqrt{3} / 2$ by using only integers. To this end we define the symbols

$$
X_{\omega}=\left[I_{3} \delta\right] q_{2}+\left[I_{3} \delta^{2}\right] q_{3}, \quad Y_{\omega}=\left[I_{3} \delta\right] q_{2}-\left[I_{3} \delta^{2}\right] q_{3},
$$

where $I_{3}$ is an arbitrary but fixed integer. Here $\omega=\left(\xi_{\omega}, \eta_{\omega}\right)$ is the puncture of any $\Omega=\left(q_{1}+q_{2} \delta+q_{3} \delta^{2}\right) / \sigma_{r} \in R_{r}$.

Now $\xi_{\psi}=3 X_{\psi} / 2 I_{3} \sigma_{r}+\gamma$, where $|\gamma|<3\left(\left|t_{2}\right|+\left|t_{3}\right|\right) / 2 I_{3} \sigma_{r}$. If $I_{3}>150 \delta^{2}$, we see from (6.5) that $|\gamma|<1 / 25$; thus, when $4 X_{\psi}>\sigma_{r} I_{3}$, we have $3 X_{\psi} / 2 I_{3} \sigma_{r}>$ $3 / 8$ and $\xi_{\psi}>.256$. Also, when $4 X_{\psi}<\sigma_{r} I_{3}$, we have $\xi_{\psi}<1 / 2\left(I_{3}>150 \delta^{2}\right)$.

To determine whether or not $\left|\eta_{\psi}\right|<\sqrt{3} / 2$ is somewhat more difficult, we first note that $\left|\eta_{\psi}\right|<\sqrt{3} / 2$ if and only if $\epsilon=\operatorname{sgn}\left(\sigma_{r}-\left|\delta t_{1}-\delta^{2} t_{2}\right|\right)>0$. From (6.5) we have $\left|t_{2}\right|+\delta\left|t_{3}\right|<4 \delta^{2}$ and $3.1 \delta^{2}\left(\left|t_{2}\right|+\left|t_{3}\right|\right) f\left(t_{2}, t_{3}\right)<3.1 \delta^{2}\left(4 \delta^{2}\right)^{3}<200 \delta^{8}$. It follows from Lemma 7.3 that $\operatorname{sgn}\left(I_{3} \sigma_{r}-\left|Y_{\psi}\right|\right)=\epsilon$ when $I_{3}>200 \delta^{8}$. It should also be noted here that if $\Omega=\theta_{g}^{(r)}$, then from (6.8) we get $\left|q_{2}\right|+\left|q_{3}\right| \delta<$ $(2+2 \sqrt{3}) \delta^{2}$ and

$$
506 \delta^{8}>3.1(2+2 \sqrt{3})^{3} \delta^{8}>3.1 \delta^{2}\left(\left|q_{2}\right|+\left|q_{3}\right|\right) f\left(q_{2}, q_{3}\right) .
$$


Hence, we can determine whether or not $\left|\eta_{\omega}\right|<\sqrt{3} / 2$ by checking whether or not $\left|Y_{\omega}\right|<\sigma_{r} I_{3}$.

We now have

Lemma 9.1. Let $S$ be defined as in Section 5. Then, if $I_{3}>200 \delta^{8}, S$ is given by Table 4 below.

TABLE 4

\begin{tabular}{lc}
\hline \multicolumn{2}{c}{$\begin{array}{c}\text { restrictions on } \\
X_{\psi} \text { and } Y_{\psi}\end{array}$} \\
\hline$\left|Y_{\psi}\right|<\sigma_{r} I_{3}$ & $\{(1,0),(0,1),(1,-1)\}$ \\
\hline $4\left|X_{\psi}\right|>\sigma_{r} I_{3}$ & $\{(1,0),(0,1),(1,-1),(1,1)\}$ \\
\hline$\left|Y_{\psi}\right|>\sigma_{r} I_{3}, 4\left|X_{\psi}\right|<\sigma_{r} I_{3}$ & $\{(1,0),(1,-1),(1,1),(2,1)\}$ \\
\hline
\end{tabular}

Proof. Follows from the above remarks and Table 2 of Section 5.

We must now show how to find that element of $S$ to which the puncture of $\theta_{g}^{(r)}$ corresponds. If $\Omega=a \Phi+b \Psi+c=\left(q_{1}+q_{2} \delta+q_{3} \delta^{2}\right) / \sigma_{r}$, then $q_{1}=\sigma_{r} c+q$, $q=a s_{1}+b t_{1}, q_{2}=a s_{2}+b t_{2}$, and $q_{3}=a s_{3}+b t_{3}$. Also

$$
\left\{\begin{aligned}
{\left[-a \zeta_{\Phi}-b \zeta_{\Psi}\right] } & =\left[\left(-2 q+q_{2} \delta+q_{3} \delta^{2}\right) / 2 \sigma_{r}\right] \\
& =\left[\left(-2 q+\left[q_{2} \delta+q_{3} \delta^{2}\right]\right) / 2 \sigma_{r}\right] \\
{\left[1 / 2-a \zeta_{\Phi}-b \zeta_{\Psi}\right] } & =\left[\left(\sigma_{r}-2 q+q_{2} \delta+q_{3} \delta^{2}\right) / 2 \sigma_{r}\right] \\
& =\left[\left(\sigma_{r}-2 q+\left[q_{2} \delta+q_{3} \delta^{2}\right]\right) / 2 \sigma_{r}\right]
\end{aligned}\right.
$$

We now prove

LEMMA 9.2. If $r, s, t \in Z$ and $s$ and $t$ are not both zero, then

$$
\left[r+s \delta+t \delta^{2}\right]=\left[\left(r I+s[\delta I]+t\left[\delta^{2} I\right]\right) / I\right],
$$

when $I>3.1 \delta^{2}(|s|+|t|) f(s, t)$.

Proof. In the inequality $\left|r+s \delta+t \delta^{2}\right|>1 / 3.1 \delta^{2} f(s, t)$ of Lemma 7.2, we note that the right-hand side is independent of $r$. If we put $T=s \delta+t \delta^{2}$ and select $r$ such that $r+T=\{T\}$, then $\{T\}>1 / 3.1 \delta^{2} f(s, t)$. If we select $r$ such that $r+T=$ $1-\{T\}$, then $1-\{T\}>1 / 3.1 \delta^{2} f(s, t)$.

If we put $A=\left(s[\delta I]+t\left[\delta^{2} I\right]\right) / I$, then

$$
|A-T|<(|s|+|t|) / I<1 / 3.1 \delta^{2} f(s, t) .
$$

Hence, $|A-T|<\min (\{T\}, 1-\{T\})$ and we have $[T]<A<[T]+1$. The lemma now follows easily. 
Now if $\Omega=\theta_{g}^{(r)}$, then $\Omega^{*}=\Omega$; thus by the remarks in Section 4 , formulas (9.1) and (9.2) and Lemma 9.2, we have $c=l$ or possibly $l+1$, where

$$
l= \begin{cases}{\left[\left(\left[X_{\omega} / I_{3}\right]-2 q\right) / 2 \sigma_{r}\right]} & \left(\left|Y_{\omega}\right|<\sigma_{r} I_{3}\right), \\ {\left[\left(\sigma_{r}-2 q+\left[X_{\omega} / I_{3}\right]\right) / 2 \sigma_{r}\right]} & \left(\left|Y_{\omega}\right|>\sigma_{r} I_{3}\right),\end{cases}
$$

and $I_{3}>506 \delta^{8}$. Further $c$ can be $l+1$ only when $\left|Y_{\omega}\right|<\sigma_{r} I_{3}$ and

$$
\left(2 \sigma_{r} l+2 q-q_{2} \delta-q_{3} \delta^{2}\right) / 2 \sigma_{r}<-1 / 2 .
$$

By (9.1) and Lemma 7.3, we see that (9.4) is true if and only if

$$
X_{\omega}-2 I_{3} q>I_{3} \sigma_{r}+2 I_{3} \sigma_{r} l .
$$

If, for each element of the set $\{a \Phi+b \Psi \mid(a, b) \in S\}$, we calculate possible values for $c$ and $\Omega=c+a \Phi+b \Psi$ by using the formulas (9.3) and (9.5) above with $I_{3}>506 \delta^{8}$, we get a set $P=\left\{\Omega_{1}, \Omega_{2}, \Omega_{3}, \ldots, \Omega_{k}\right\}$, where $k \leqslant 8$ and $\Omega_{i}=\left(q_{1 i}+q_{2 i} \delta+q_{3 i} \delta^{2}\right) / \sigma_{r}$. One of these $\Omega_{i}$ is $\theta_{g}^{(r)}$. Further, if $\Omega=$ $\left(q_{1}+q_{2} \delta+q_{3} \delta^{2}\right) / \sigma_{r}$ is any element of this set, then by (6.5),

$$
\left|q_{2}\right|+\left|q_{3}\right| \delta \leqslant a\left(\left|s_{2}\right|+\left|s_{3}\right| \delta\right)+b\left(\left|t_{2}\right|+\left|t_{3}\right| \delta\right)<12 \delta^{2}
$$

and

$$
\left|X_{\omega} / I_{3}-q_{2} \delta-q_{3} \delta^{2}\right|<\left(\left|q_{2}\right|+\left|q_{3}\right|\right) / I_{3}<1 .
$$

Since $-2<[r]-s<1$ when $|r-s|<1$, we see, on putting $r=\left(\left[X_{\omega} / I_{3}\right]-2 q\right) / 2 \sigma_{r}$, $s=\left(-2 q+q_{2} \delta+q_{3} \delta^{2}\right) / 2 \sigma_{2}$, that $\left|\zeta_{\Omega}\right|<2$. Since $q_{1} / \sigma_{r}=\zeta_{\Omega}+\xi_{\omega} / 3$, we have $\left|q_{1}\right|<2 \sigma_{r}+12 \delta^{3} / 2$ from (9.6) and, therefore,

$$
\left|q_{1}\right|<12 D, \quad\left|q_{2}\right|<12 \delta^{2}, \quad\left|q_{3}\right|<12 \delta .
$$

For each $\Omega_{i}=\left(q_{1 i}+q_{2 i} \delta+q_{3 i} \delta^{2}\right) / \sigma_{r} \in P$ define $W_{i}=q_{1 i} I_{3}+q_{2 i}\left[I_{3} \delta\right]+$ $q_{3 i}\left[I_{3} \delta^{2}\right]$, and let $W=\left\{W_{i} \mid \Omega_{i} \in P\right\}$. We now prove

LEмmA 9.3. If $W_{j}$ is the least element of $W$ such that $\Omega_{j} \in \mathcal{C}$, then $\theta_{g}^{(r)}=\Omega_{j}$.

Proof. If $\Omega \in C \cap P$, then $\zeta_{\Omega}>-1$ and $\xi_{\omega}>0$; hence $\Omega>-1$, and, since 1 is a relative minimum of $R_{r}$, we must have $\Omega>1$. It follows that $\theta_{g}^{(r)}$ is the least element of $C \cap P$.

Suppose $\Omega_{i}=\theta_{g}^{(r)} \neq \Omega_{j}$; then $X=\left(x_{1}+x_{2} \delta+x_{3} \delta^{2}\right) / \sigma_{r}=\Omega_{j}-\Omega_{i}>0$. Further, $\left|x_{2}\right|+\delta\left|x_{3}\right|<\left|m_{2}\right|+\left|m_{3}\right| \delta+\left|q_{2 j}\right|+\left|q_{3 j}\right| \delta<(2+2 \sqrt{3}) \delta^{2}+12 \delta^{2}<$ $18 \delta^{2}$ by (6.8) and (9.6). If $\chi$ is the puncture of $X$, we see that, since $\Omega_{i}, \Omega_{j} \in \mathcal{C}$, we have $\left|\zeta_{X}\right|,\left|\eta_{\chi}\right|<2$. Now $X \in R_{r}$ and $\sigma_{1} \sigma_{r}\left|e_{r}\right| \mid N\left(\sigma_{r} X\right)$ by Theorem 3.2; hence,

$$
N(X)=|X|\left(\eta_{X}^{2}+\zeta_{X}^{2}\right)>\sigma_{1}\left|e_{r}\right| / \sigma_{r}^{2}=1 / Q_{r}>1 / 3 D
$$

by (3.1) and (6.4). Since $\eta_{X}^{2}+\zeta_{X}^{2}<8$, we find that $|X|>1 / 24 D$ and

$$
I_{3} \sigma_{r}|X|>506 \delta^{8} \sigma_{r} / 24 D>18 \delta^{2}>\left|x_{2}\right|+\left|x_{3}\right| \delta>\left|I_{3} \sigma_{r} X-W_{j}+W_{i}\right|
$$

thus, $\operatorname{sgn}(X)=\operatorname{sgn}\left(w_{j}-w_{i}\right)$. Since $X>0$ and $W_{j}-W_{i} \leqslant 0$ (by definition of $\Omega_{j}$ ), we have a contradiction. 
It remains to devise a technique, which uses integers only, to determine when $\Omega \in \mathcal{C}$. We do this in

THEOREM 9.4. If $q_{1}+q_{2}\left[I_{3} \delta\right]+q_{3}\left[I_{3} \delta^{2}\right]$ is the least element of $W$ such that

$$
\sigma_{1}^{2} Q_{r}^{2}>3 \tau_{r}\left(\sigma_{r} q_{1}^{\prime} /\left|e_{r}\right|-q_{1} \Sigma\right)+\Sigma^{2}
$$

where $\tau_{r}=\sigma_{r} /\left|e_{r}\right|, q_{1}^{\prime}=q_{1}^{2}-D q_{2} q_{3}, q_{2}^{\prime}=D q_{3}^{2}-q_{1} q_{2}, q_{3}^{\prime}=q_{2}^{2}-q_{1} q_{3}$, and $\Sigma=$ $q_{1}^{\prime} q_{1}+D\left(q_{2} q_{3}^{\prime}+q_{3} q_{2}^{\prime}\right) / l e_{r} \mid \sigma_{r}$, then $\theta_{g}^{(r)}=\left(q_{1}+q_{2} \delta+q_{3} \delta^{2}\right) / \sigma_{r}$.

Proof. If $\Omega \in R_{r}$, then $\Omega \in C$ if and only if $\Omega^{\prime} \Omega^{\prime \prime}<1$. But $\Omega^{\prime} \Omega^{\prime \prime}<1$ if and only if $\Omega N(\Omega)<\Omega^{2}$ or $N(\Omega) N\left(\Omega-N(\Omega)\right.$ ) $=N\left(\Omega^{2}-\Omega N(\Omega)\right.$ ) $>0$ (since $A$ and $N(A)$ have the same sign). It now suffices to note that

$$
\begin{aligned}
N(\Omega-N(\Omega)) & =N(\Omega)\left(1-\operatorname{Tr}\left(\Omega \Omega^{\prime}\right)+N(\Omega) \operatorname{Tr}(\Omega)-N(\Omega)^{2}\right) \\
& =\frac{N(\Omega)}{\sigma_{r}^{4}}\left(\sigma_{r}^{4}-3 q_{1}^{\prime} \sigma_{r}^{2}+3 \sigma_{r} q_{1} N(\Omega) \sigma_{r}^{2}-N(\Omega)^{2} \sigma_{r}^{4}\right) \\
& =\frac{N(\Omega) e_{r}^{2}}{\sigma_{r}^{4}}\left(\sigma_{1}^{2} Q_{r}^{2}-3 \tau_{r}\left(\sigma_{r} q_{1}^{\prime} /\left|e_{r}\right|-q_{1} \Sigma\right)-\Sigma^{2}\right),
\end{aligned}
$$

from (3.1).

Note that from Theorem 3.2 both $\Sigma$ and $q_{1}^{\prime} / / e_{r} \mid$ are integers. Also, if $\Omega=\theta_{g}^{(r)}$, then

$$
\Sigma=\sigma_{r}^{2} N\left(\dot{\theta}_{g}^{(r)}\right) /\left|e_{r}\right|=\sigma_{r+1}^{2} /\left|e_{r+1}\right|=\sigma_{1} Q_{r+1}<3 D
$$

by Theorem 3.3, (3.1) and (6.4).

10. The Final Algorithm. We are now able to present the algorithm for determining $R$ for $Q(\sqrt[3]{D})$ in its entirety. We first assume that $\delta=\sqrt[3]{D}$ has been calculated with sufficient accuracy that we can determine

$$
\begin{gathered}
d_{1}=\left[I_{1} \delta\right], \quad d_{2}=\left[\sqrt{3} I_{2} \delta\right], \quad d_{3}=\left[\sqrt{3} I_{2} \delta^{2}\right], \quad d_{4}=\left[I_{3} \delta\right], \\
d_{5}=\left[I_{3} \delta^{2}\right], \quad \text { where } I_{1}=\left[841 \delta^{2} D\right]+1, I_{2}=\left[80 \delta^{2} D\right]+1, I_{3}=\left[506 D^{2} \delta^{2}\right]+1
\end{gathered}
$$

In practice this is easily done by using Newton's method to determine $\delta$. We also determine $g_{2}$ such that $D=g_{1} g_{2}^{2},\left(g_{1}, g_{2}\right)=1$ and $g_{1}, g_{2}$ are square-free.

When $D \equiv \pm 1(\bmod 9)$, we put $m_{1}=0, m_{2}=3 g_{2}, m_{3}=0, n_{1}=g_{2}^{2}, n_{2}=$ $\pm g_{2}^{2}, n_{3}=1, \sigma=\sigma_{1}=3 g_{2}, e=e_{1}=3 g_{2}$; otherwise, put $m_{1}=0, m_{2}=g_{2}, m_{3}=0$, $n_{1}=0, n_{2}=0, n_{3}=1, \sigma_{1}=\sigma=e=e_{1}=g_{2}$. We also initialize the value of $r$ to be 1 and that of $R$ to be 0 .

\section{Algorithm.}

(I) Calculate, by using Algorithm $\mathrm{B}$, the coefficients $s_{1}, s_{2}, s_{3}, t_{1}, t_{2}, t_{3}$ of the elements $\Phi$ and $\Psi$ of the $\beta$-basis $[1, \Phi, \Psi]$ of $R_{r}$.

(II) Determine $\theta_{g}^{(r)}, \theta_{h}^{(r)}$ and increase $R$ by $\log \theta_{g}^{(r)}$. Calculate the coefficients $m_{1}, m_{2}, m_{3}, n_{1}, n_{2}, n_{3}$ and $\sigma_{r+1}$ of the basis $\left[1,1 / \theta_{g}^{(r)}\right.$, $\left.\theta_{h}^{(r)} / \theta_{g}^{(r)}\right]$ of $R_{r+1}$. 
(III) Find $e_{r+1}=m_{2} n_{3}-n_{2} m_{3}$. If $\sigma_{r+1}=\sigma$ and $\left|e_{r+1}\right|=e$, terminate the algorithm; otherwise increase $r$ by 1 and return to (I).

We now describe the algorithms of I and II in more detail. We define as above

$$
\begin{aligned}
& x_{\mu}=I_{1} m_{2}+d_{1} m_{3}, \quad y_{\mu}=I_{1} m_{2}-d_{1} m_{3}, \quad x_{\nu}=I_{1} n_{2}+d_{1} n_{3} \text {, } \\
& y_{\nu}=I_{1} n_{2}-d_{1} n_{3}, \quad \bar{y}_{\mu}=d_{2} m_{2}-d_{3} m_{3}, \quad \bar{y}_{\nu}=d_{2} n_{2}-d_{3} n_{3} \text {. }
\end{aligned}
$$

\section{Algorithm I.}

(i) Transform the basis of $R_{r}$ by $K_{2}(-k, 1)$, where $k=\left[n_{3} / m_{3}\right]$ when $m_{3} \neq 0$ or $k=\left[n_{2} / m_{2}\right]$ when $m_{3}=0$.

(ii) Transform the basis by $\left(\begin{array}{cc}k_{1} & 0 \\ 0 & k_{2}\end{array}\right)$, where $k_{1}=\operatorname{sgn}\left(x_{\mu}\right), k_{2}=\operatorname{sgn}\left(x_{\nu}\right)$.

(iii) If $x_{\nu}<x_{\mu}$, transform the basis by $K_{2}(1,1)$ and go to (iv) unless $y_{\nu} y_{\mu}<0$ and $\left|y_{\nu}\right|>\left|y_{\mu}\right|$. If this latter case occurs transform the basis by $K_{1}(0,1)$ instead of $K_{2}(1,1)$ and go to (vi).

(iv) If $y_{\mu} y_{\nu}<0$ go directly to step (v); otherwise,

(1) if $\left[y_{\nu} / y_{\mu}\right]=\left[x_{\nu} / x_{\mu}\right]=k$, transform the basis by $K_{1}(-k, 1)$ until $\left[y_{\nu} / y_{\mu}\right] \neq\left[x_{\nu} / x_{\mu}\right]$. When we find a basis such that $\left[y_{\nu} / y_{\mu}\right] \neq\left[x_{\nu} / x_{\mu}\right]$ we execute one of the following steps.

(2) If $\left[x_{\nu} / x_{\mu}\right]+1=\left[y_{\nu} / y_{\mu}\right]=k$, transform the basis by $K_{1}(k,-1)$ and go to $(\mathrm{v})$.

(3) If $k=\left[x_{\nu} / x_{\mu}\right]=\left[y_{\nu} / y_{\mu}\right]+1$, transform the basis by $K_{1}(-k, 1)$ and go to $(\mathrm{v})$.

(4) If $\left[x_{\nu} / x_{\mu}\right]<\left[y_{\nu} / y_{\mu}\right]-1$, then transform the basis by $K_{1}\left(\left[x_{\nu} / x_{\mu}\right]+1,-1\right)$ when $\left|\bar{y}_{\mu}\right|<I_{2} \sigma_{r}$; otherwise, transform by $K_{2}\left(\left[y_{\nu} / y_{\mu}\right],-1\right)$. Go to (vi).

(5) If $\left[x_{\nu} / x_{\mu}\right]>\left[y_{\nu} / y_{\mu}\right]+1$, transform the basis by $K_{1}\left(-\left[x_{\nu} / x_{\mu}\right], 1\right)$ when $\left|\bar{y}_{\mu}\right|<I_{2} \sigma_{r}$; otherwise, transform by $K_{2}\left(-\left[y_{\nu} / y_{\mu}\right]-1,1\right)$. Go to (vi).

(v) If $\left|y_{\mu}\right|>\left|y_{\nu}\right|$, go to (vi). If $\left|y_{\mu}\right| \leqslant\left|y_{\nu}\right|$, transform the basis by $K_{1}\left(-\left[x_{\nu} / x_{\mu}\right], 1\right)$ when $\left|\bar{y}_{\mu}\right|<I_{2} \sigma_{r}$; otherwise transform by $K_{2}\left(\left[-y_{\nu} / y_{\mu}\right], 1\right)$.

(vi) If $\left|\bar{y}_{\mu}\right| \geqslant I_{2} \sigma_{r}$ and $\left|\bar{y}_{\nu}\right| \leqslant I_{2} \sigma_{r}$, terminate Algorithm I. If $\left|\bar{y}_{\mu}\right|<$ $I_{2} \sigma_{r}$ execute step (1) below; otherwise execute step (2).

(1) Transform the basis by $K_{1}(-k, 1)$, where $k=\left[x_{\nu} / x_{\mu}\right]$. Repeat this process until $\left|\bar{y}_{\mu}\right| \geqslant I_{2} \sigma_{r}$; at this point terminate Algorithm I.

(2) Transform the basis by $K_{3}(1, k)$, where $k=\left[-y_{\mu} / y_{\nu}\right]$. Repeat this process until $\left|\bar{y}_{\nu}\right| \leqslant I_{2} \sigma_{r}$; at this point terminate Algorithm I.

Note that in the process of making all the calculations needed to execute Algorithm I we never need an integer larger than $\max \left(n_{2}, m_{2}, n_{3}, m_{3}\right) I_{1}$. However, this is always less than $\left(\left|e_{r}\right|+(1+\sqrt{5}) \delta^{2}\right) I_{1}<\left(3 D+(1+\sqrt{5}) \delta^{2}\right) I_{1}$. Also, if we did not wish to carry the values of $m_{1}$ and $n_{1}$ during these calculations we could still 
determine $s_{1}$ and $t_{1}$ by using the formulas

$$
e_{r} t_{1}=k_{11} m_{1}+k_{12} n_{1}, \quad e_{r} s_{1}=k_{21} m_{1}+k_{22} n_{1},
$$

where

$$
k_{11}=\left|\begin{array}{ll}
t_{2} & n_{2}^{*} \\
t_{3} & n_{3}^{*}
\end{array}\right|, \quad k_{12}=\left|\begin{array}{ll}
m_{2}^{*} & t_{2} \\
m_{3}^{*} & t_{3}
\end{array}\right|, \quad k_{21}=\left|\begin{array}{ll}
s_{2} & n_{2}^{*} \\
s_{3} & n_{3}^{*}
\end{array}\right|, \quad k_{22}=\left|\begin{array}{ll}
m_{2}^{*} & s_{2} \\
m_{3}^{*} & s_{3}
\end{array}\right|
$$

and $n_{2}^{*}, n_{3}^{*}, m_{2}^{*}, m_{3}^{*}$ are, respectively, the values of $n_{2}, n_{3}, m_{2}, m_{3}$ before Algorithm $\mathrm{I}$ is executed. In order to ensure that $s_{1}$ and $t_{1}$ are not too large, we can reduce them modulo $\sigma_{r}$.

Algorithm II.

(i) Initialization. Put $\tau_{r}=\sigma_{r} /\left|e_{r}\right|, Q=\sigma_{r} \tau_{r}$. Calculate $s_{2} d_{4}, s_{3} d_{5}$, $t_{2} d_{4}, t_{3} d_{5}, Q^{2}, \sigma_{r} I_{3}, X_{\phi}=s_{2} d_{4}+s_{3} d_{5}, Y_{\phi}=s_{2} d_{4}-s_{3} d_{5}, X_{\psi}=$ $t_{2} d_{4}+t_{3} d_{5}, Y_{\psi}=t_{2} d_{4}-t_{3} d_{5}$.

(ii) If $\left|Y_{\psi}\right|<\sigma_{r} I_{3}$, put $k=3$ and $\left(a_{1}, b_{1}\right)=(1,0),\left(a_{2}, b_{2}\right)=(0,1)$, $\left(a_{3}, b_{3}\right)=(1,-1)$. When $\left|Y_{\psi}\right|>\sigma_{r} I_{3}$, put $k=4$. If $4\left|X_{\psi}\right|<$ $\sigma_{r} I_{3}$, put $\left(a_{1}, b_{1}\right)=(1,0),\left(a_{2}, b_{2}\right)=(0,1),\left(a_{3}, b_{3}\right)=(1,-1)$, $\left(a_{4}, b_{4}\right)=(1,1)$; otherwise put $\left(a_{1}, b_{1}\right)=(1,0),\left(a_{2}, b_{2}\right)=(1,-1)$, $\left(a_{3}, b_{3}\right)=(1,1)$ and $\left(a_{4}, b_{4}\right)=(2,1)$.

(iii) For each pair $\left(a_{i}, b_{i}\right)$ calculate $q_{i}=a_{i} s_{1}+b_{i} t_{1}, X_{i}=a_{i} X_{\phi}+b_{i} X_{\psi}$, $Y_{i}=a_{i} Y_{\phi}+b_{i} Y_{\psi}$. If $\left|Y_{i}\right|<\sigma_{r} I_{3}$, put

$$
l_{1 i}=\left[\frac{\left[X_{i} / I_{3}\right]-2 q_{i}}{2 \sigma_{r}}\right], \quad q_{1 i}=q_{i}+\sigma_{r} l_{1 i}
$$

If $X_{i}-2 I_{3} q_{i}>2 \sigma_{r} I_{3} l_{1 i}+\sigma_{r} I_{3}$, put

$$
l_{2 i}=l_{1 i}+1, \quad q_{2 i}=q_{1 i}+\sigma_{r} .
$$

If $\left|Y_{i}\right|>\sigma_{r} I_{3}$, put

$$
l_{1 i}=\left[\frac{\sigma_{r}-2 q_{i}+\left[X_{i} / I_{3}\right]}{2 \sigma_{r}}\right], \quad q_{1 i}=q_{i}+\sigma_{r} l_{1 i} .
$$

Let $W=\left\{I_{3} q_{j i}+X_{i} \mid i \leqslant k, j \leqslant 2\right\}$. Since $t_{1}$ and $s_{1}$ have been reduced modulo $\sigma_{r}, q_{i}<3 \max \left(t_{1}, s_{1}\right)<9 D$ and $\left|2 \sigma_{r} I_{3} l_{1 i}\right|<\left|X_{i}\right|+\left|2 q_{i} I_{3}\right|+2 \sigma_{r} I_{3}$, we see by (9.6) that the largest integer needed in the calculations of Algorithm II to this point is less than $\left(24 D+13 \delta^{2}\right) I_{3}$.

(iv) Find the minimum $I_{3} q_{j k}+X_{\kappa}$ of the set $W$. Put $m_{1}=q_{j k}, m_{2}=$ $a_{\kappa} s_{2}+b_{\kappa} t_{2}, m_{3}=a_{\kappa} s_{3}+b_{\kappa} t_{3}$ and calculate the integers (Theorem 3.2)

$$
\begin{gathered}
\bar{m}_{1}=\left(m_{1}^{2}-D m_{2} m_{3}\right) /\left|e_{r}\right|, \quad \bar{m}_{2}=\left(D m_{3}^{2}-m_{1} m_{2}\right) /\left|e_{r}\right|, \quad \bar{m}_{3}=\left(m_{2}^{2}-m_{1} m_{3}\right) /\left|e_{r}\right| \\
\Sigma=\left(m_{1} \bar{m}_{1}+D\left(m_{2} \bar{m}_{3}+m_{3} \bar{m}_{2}\right)\right) / \sigma_{r} .
\end{gathered}
$$


If either

$$
\begin{gathered}
\left|\bar{m}_{1}\right|>[(1+\sqrt{5}) \sqrt{3 D} D], \quad\left|\bar{m}_{2}\right|>\left[(1+\sqrt{5}) \sqrt{3 D} \delta^{2}\right], \\
\left|\bar{m}_{3}\right|>[(1+\sqrt{5}) \sqrt{3 D} \delta], \quad \text { or } \Sigma>3 D,
\end{gathered}
$$

then by (6.13) or (9.8), $\left(m_{1}+m_{2} \delta+m_{3} \delta^{2}\right) / \sigma_{r} \neq \theta_{g}^{(r)}$ and we eliminate $I_{3} q_{j \kappa}+X_{\kappa}$ from $W$ and return to the beginning of step

(iv). (This step is optional. It simply ensures that the numbers $\bar{m}_{1}, \bar{m}_{2}, \bar{m}_{3}$ and $\Sigma$ do not get too large.)

If $\Sigma^{2}+3 \tau_{r}\left(\bar{m}_{1} \sigma_{r}-m_{1} \Sigma\right)>Q^{2}$, eliminate $I_{3} q_{j \kappa}+X_{\kappa}$ from $W$ and return to step (iv); otherwise, put $n_{1}=t_{1}, n_{2}=t_{2}, n_{3}=t_{3}$ when $k=1$ or $n_{1}=s_{1}, n_{2}=s_{2}, n_{3}=s_{3}$ when $\kappa \neq 1$.

(v) Find $d=$ g.c.d. $\left(\bar{m}_{1}, \bar{m}_{2}, \bar{m}_{3}\right)$ and replace the values of $\bar{m}_{i}$ by those of $\bar{m}_{i} / d(i=1,2,3)$. Calculate $\bar{n}_{1}=n_{1} \bar{m}_{1}+D\left(n_{3} \bar{m}_{2}+n_{2} \bar{m}_{3}\right), \bar{n}_{2}=$ $n_{2} \bar{m}_{1}+n_{1} \bar{m}_{2}+D n_{3} \bar{m}_{3}, \bar{n}_{3}=n_{3} \bar{m}_{1}+n_{2} \bar{m}_{2}+n_{1} \bar{m}_{3}, \bar{d}=$ g.c.d. $\left(\bar{n}_{1}, \bar{n}_{2}, \bar{n}_{3}, \sigma_{r}\right), \bar{\sigma}_{r}=\sigma_{r} / \bar{d}$.

Put $m_{i}=\bar{\sigma}_{r} \bar{m}_{i}, n_{i}=\bar{n}_{i} / \bar{d},(i=1,2,3), \sigma_{r+1}=\Sigma / d \bar{d}$.

From (6.6), (6.11), (6.12) and the fact that $n_{1}<\sigma_{r}<3 D$, we see that the numbers in (v) never exceed $36 D^{2}$. Also, in step (iv), by (9.7), $\left|m_{1}\right|<12 D,\left|m_{2}\right|$ $<12 \delta^{2},\left|m_{3}\right|<12 \delta$; thus, we have $\left|\bar{m}_{1}\right|,\left|\bar{m}_{2}\right|,\left|\bar{m}_{3}\right|<288 D^{2}$.

11. Implementation of the Algorithms. Programs implementing the algorithms described above were written in Assembler Language for an IBM 370-168 computer. Use was made of double-precision arithmetic in all steps of the algorithms except in steps (i), (ii), (iii) and part of step (iv) of Algorithm II, where some extended-precision arithmetic was necessary. In the process of running these programs it was found that the speed of the algorithm could be improved by making the following alterations.

(1) When $D \geqslant 579$ check in step (iv) of Algorithm II whether or not

$$
\left|Y_{K}\right|>\left[\frac{2 \sqrt{3}}{3} \sigma_{r} I_{3}\right]+\left[5 \delta^{2}\right]
$$

before calculating $\bar{m}_{1}, \bar{m}_{2}, \bar{m}_{3}$. If $\theta_{g}^{(r)}=\Omega_{\kappa}=\left(m_{1}+m_{2} \delta+m_{3} \delta^{2}\right) / \sigma_{r}$, then from (6.9) $\left|m_{2}\right|+\left|m_{3}\right|<(2 \sqrt{3}+1) \delta^{2}+(2 \sqrt{3}+1) \delta<5 \delta^{2}$. Thus, if

$$
\left|Y_{\kappa}\right|>\left[\frac{2 \sqrt{3}}{3} \sigma_{r} I_{3}\right]+\left[5 \delta^{2}\right] \text {, }
$$

then

$$
\left|Y_{\kappa}\right|>\frac{2 \sqrt{3}}{3} \sigma_{r} I_{3}+\left|m_{2}\right|+\left|m_{3}\right| .
$$

Since $\left|2 \sigma_{r} \eta / \sqrt{3}-Y / I_{3}\right|<\left(\left|m_{2}\right|+\left|m_{3}\right|\right) / I_{3}$, where $(\xi, \eta)$ is the puncture of $\Omega_{\kappa}$, we see that $|\eta|>1$. It follows that $\theta_{g}^{(r)} \neq \Omega_{\kappa}$, and we can eliminate $I_{3} q_{j \kappa}+X_{\kappa}$ from $W$.

(2) Calculate $R$ by adding $\log \left(\theta_{g}^{(k)} \theta_{g}^{(k+1)} \theta_{g}^{(k+2)} \cdots \theta_{g}^{(k+15)}\right)$ instead of $\sum_{i=0}^{15} \log \theta_{g}^{(k+i)}$. This decreases the use of the expensive routine to calculate $\log x$.

With these improvements the computer was able to calculate $\left[1, M_{r+1}, N_{r+1}\right]$ from $\left[1, M_{r}, N_{r}\right]$ in less than $500 \mu$ seconds. This improved the speed of our previous 
algorithm [2] by a factor of 10. Much, but not all, of this increase is due to the change in computer language from FORTRAN $H$ to Assembler Language.

These programs were run for all $8984 Q(\delta)$ such that $D<10^{5}$ and the class number of $Q(\delta)$ is not divisible by three. These fields are those for which (Honda [4]) $D$ has the following values:

(i) $D=3$,

(ii) $D=p, p \equiv-1(\bmod 3)$,

(iii) $D=3 p$ or $9 p$ where $p \equiv 2,5(\bmod 9)$,

(iv) $D=p q$, where $p \equiv 2, q \equiv 5(\bmod 9)$,

(v) $D=p^{2} q$, where $p \equiv q \equiv 2,5(\bmod 9)(p<q)$.

Here $p, q$ are primes. After the regulators were calculated, the class numbers $h(D)$ were evaluated by using the method of [8]. In the tables below we give some of the results of these calculations.

In Table 5 we give each class number $h$ found, together with the frequency $f(h)$ with which $h$ occurred, the percentage $100 f(h) / 8984$, and least $D$ value such that $Q(\delta)$ has class number $h$.

\section{TABLE 5}

$\begin{array}{rr}\text { h } & f(h) \\ 1 & 4537 \\ 2 & 2132 \\ 4 & 882 \\ 5 & 258 \\ 7 & 160 \\ 8 & 297 \\ 10 & 102 \\ 11 & 38 \\ 13 & 29 \\ 14 & 49 \\ 16 & 96 \\ 17 & 14 \\ 19 & 16 \\ 20 & 54 \\ 22 & 22 \\ 23 & 7 \\ 25 & 8 \\ 26 & 11 \\ 28 & 34 \\ 29 & 6 \\ 31 & 2 \\ 32 & 19 \\ 34 & 10 \\ 35 & 3 \\ 37 & 6 \\ 38 & 5 \\ 40 & 17 \\ 41 & 3 \\ 43 & 5 \\ 44 & 9 \\ 46 & 2 \\ 49 & 5 \\ 50 & 6 \\ 52 & 6 \\ 53 & 11 \\ 56 & \end{array}$

\begin{tabular}{cr}
$100 f(h) / 8984$ & \multicolumn{1}{c}{$D$} \\
50.50 & 2 \\
23.73 & 11 \\
9.82 & 113 \\
2.87 & 263 \\
1.78 & 235 \\
3.31 & 141 \\
1.14 & 303 \\
0.42 & 2348 \\
0.32 & 1049 \\
0.55 & 514 \\
1.07 & 681 \\
0.16 & 8511 \\
0.18 & 667 \\
0.60 & 761 \\
0.24 & 281 \\
0.08 & 21241 \\
0.09 & 10181 \\
0.12 & 3403 \\
0.38 & 509 \\
0.07 & 12079 \\
0.02 & 16553 \\
0.21 & 2399 \\
0.11 & 1719 \\
0.03 & 37207 \\
0.07 & 5545 \\
0.06 & 12813 \\
0.19 & 2733 \\
0.03 & 6659 \\
0.06 & 32847 \\
0.10 & 4817 \\
0.02 & 59975 \\
0.06 & 8171 \\
0.07 & 14372 \\
0.07 & 4793 \\
0.01 & 38373 \\
0.12 & 857 \\
0.02 & 6814 \\
& \\
0.03
\end{tabular}




\begin{tabular}{|c|c|}
\hline & $f(h)$ \\
\hline 59 & 1 \\
\hline 61 & 1 \\
\hline 62 & 2 \\
\hline 64 & 7 \\
\hline 65 & 1 \\
\hline $\begin{array}{l}67 \\
68\end{array}$ & 2 \\
\hline $\begin{array}{l}68 \\
70\end{array}$ & $\begin{array}{l}4 \\
3\end{array}$ \\
\hline 71 & 3 \\
\hline 74 & 1 \\
\hline 76 & 2 \\
\hline 79 & 1 \\
\hline 80 & 6 \\
\hline 83 & 1 \\
\hline 85 & 2 \\
\hline $\begin{array}{l}86 \\
89\end{array}$ & $\begin{array}{l}1 \\
1\end{array}$ \\
\hline 92 & 1 \\
\hline 95 & 3 \\
\hline 100 & 4 \\
\hline 101 & 1 \\
\hline 104 & 3 \\
\hline 110 & 2 \\
\hline 112 & 2 \\
\hline 115 & 1 \\
\hline 118 & 1 \\
\hline 121 & 1 \\
\hline 124 & 2 \\
\hline 127 & 1 \\
\hline $\begin{array}{l}128 \\
136\end{array}$ & $\begin{array}{l}1 \\
4\end{array}$ \\
\hline $\begin{array}{l}136 \\
140\end{array}$ & $\begin{array}{l}4 \\
1\end{array}$ \\
\hline 148 & 3 \\
\hline 149 & 2 \\
\hline 154 & 2 \\
\hline 155 & 1 \\
\hline 158 & 1 \\
\hline 161 & 1 \\
\hline $\begin{array}{l}170 \\
175\end{array}$ & ? \\
\hline $\begin{array}{l}175 \\
181\end{array}$ & $\begin{array}{l}1 \\
1\end{array}$ \\
\hline 191 & 1 \\
\hline 193 & $=$ \\
\hline 196 & 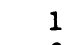 \\
\hline 200 & 2 \\
\hline 214 & 2 \\
\hline 230 & 1 \\
\hline 232 & 1 \\
\hline $\begin{array}{l}254 \\
262\end{array}$ & 1 \\
\hline $\begin{array}{l}262 \\
280\end{array}$ & $\begin{array}{l}1 \\
1\end{array}$ \\
\hline 284 & 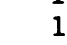 \\
\hline 296 & 1 \\
\hline 305 & 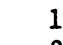 \\
\hline 316 & 2 \\
\hline $\begin{array}{l}334 \\
340\end{array}$ & $\begin{array}{l}1 \\
1\end{array}$ \\
\hline $\begin{array}{l}340 \\
352\end{array}$ & 1 \\
\hline 358 & \\
\hline 370 & \\
\hline $\begin{array}{l}389 \\
39 ?\end{array}$ & 1 \\
\hline 392 & \\
\hline
\end{tabular}

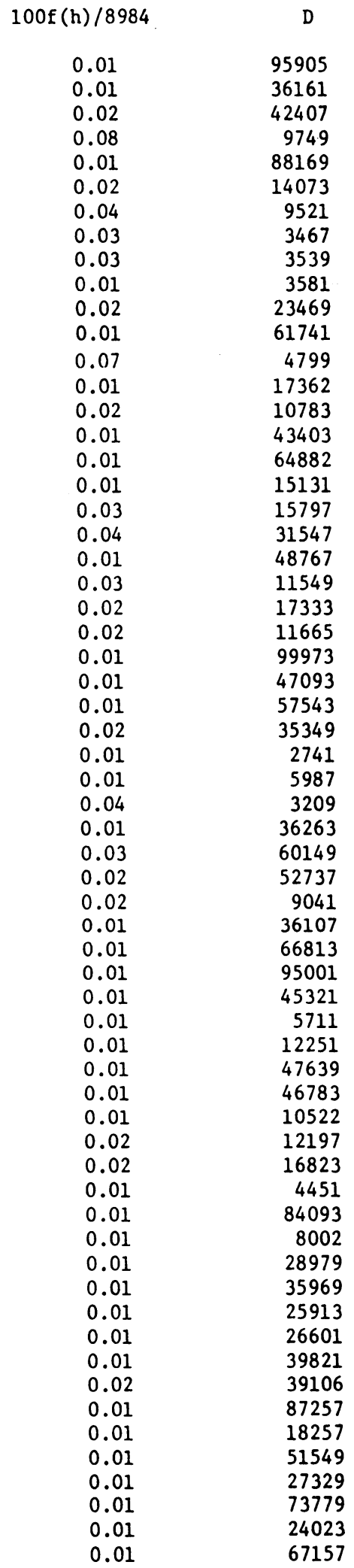




$\begin{array}{rccc}\text { h } & f(h) & 100 f(h) / 8984 & D \\ 400 & 1 & 0.01 & 53434 \\ 421 & 1 & 0.01 & 47303 \\ 433 & 1 & 0.01 & 69539 \\ 583 & 1 & 0.01 & 63766 \\ 628 & 1 & 0.01 & 61547 \\ 698 & 1 & 0.01 & 30867 \\ 706 & 1 & 0.01 & 26991 \\ 748 & 1 & 0.01 & 17573 \\ 827 & 1 & 0.01 & 97066 \\ 920 & 1 & 0.01 & 17579 \\ 980 & 1 & 0.01 & 38463 \\ 1190 & 1 & 0.01 & 74079 \\ 1442 & 1 & 0.01 & 32771 \\ 1484 & 1 & 0.01 & 79601 \\ 1640 & 1 & 0.01 & 54874 \\ 2380 & 1 & 0.01 & 54869\end{array}$

In Table 6, we give the values of $D$, the regulator $R(D)$ of $Q(\delta)$ and $J$, the length of Voronoi's algorithm period for $\delta$ such that $R(D)>R(d)$ for all $d$ such that 8429 $<d<D$ and $3 \nmid h(d)$. For the earlier part of this table see Table 5 of Barrucand, Williams and Baniuk [1].

TABLE 6

$D$
10037
10067
11621
14897
15261
15527
17669
19391
21839
22469
26417
28517
29063
32213
34607
36821
38039
39129
39521
43863
54293
55901
56993
60887
62889
66431
72227
72617
76259
84629
88661
90033
92009
96797

$\mathbf{R}(\mathrm{D})$

17941.60487

18150.81288

25661.99636

28630.01878

28634.12148

31541.56340

32388.80366

42811.86808

47361.35191

47942.75017

56816.82041

57091.82492

63398.84106

71481.68242

75693.99813

76097.18294

79677.96103

87213.59555

92172.43813

101072.02023

108016.52068

115433.63108

117983.12761

130509.10552

135188.22005

150019.35639

154817.70011

168197.50896

172072.28147

180297.38717

191840.92392

198214.96650

218706.73901

222426.50649
J

15972

16318

22908

25280

25190

27991

28517

38337

42122

42716

50385

50671

56707

63674

66931

67252

70707

77128

81615

89956

95477

102213

104106

116010

119536

133096

136734

149072

152790

159867

169795

175891

193034

197114 
Finally, in Table 7, we give the continuation of Table 3 of [8]. Here $n(x)$ is the number of primes $q(q \equiv-1(\bmod 3))$ which are less than or equal to $x$ and $g(x)$ is the number of those primes such that $h(q)=1$.

TABLE 7

\begin{tabular}{cc|cc|cc}
$\mathbf{x}$ & $100 \mathbf{g}(\mathbf{x}) / \mathrm{n}(\mathbf{x})$ & $\mathbf{x}$ & $100 \mathbf{g}(\mathbf{x}) / \mathrm{n}(\mathbf{x})$ & $\mathbf{x}$ & $100 \mathbf{g}(\mathbf{x}) / \mathrm{n}(\mathbf{x})$ \\
36000 & 47.327 & 58000 & 48.474 & 80000 & 47.984 \\
37000 & 46.910 & 59000 & 48.381 & 81000 & 48.030 \\
38000 & 46.951 & 60000 & 48.225 & 82000 & 47.978 \\
39000 & 46.945 & 61000 & 48.250 & 83000 & 47.975 \\
40000 & 47.326 & 62000 & 48.210 & 84000 & 47.959 \\
41000 & 47.190 & 63000 & 48.315 & 85000 & 47.837 \\
42000 & 47.528 & 64000 & 48.260 & 86000 & 47.824 \\
43000 & 47.184 & 65000 & 48.313 & 87000 & 47.798 \\
44000 & 47.302 & 66000 & 48.336 & 88000 & 47.810 \\
45000 & 47.595 & 67000 & 48.359 & 89000 & 47.747 \\
46000 & 47.766 & 68000 & 48.203 & 90000 & 47.818 \\
47000 & 47.826 & 69000 & 48.089 & 91000 & 47.920 \\
48000 & 47.868 & 70000 & 47.998 & 92000 & 47.883 \\
49000 & 47.866 & 71000 & 48.024 & 93000 & 47.826 \\
50000 & 47.904 & 72000 & 47.979 & 94000 & 47.781 \\
51000 & 47.922 & 73000 & 48.001 & 95000 & 47.680 \\
52000 & 47.886 & 74000 & 48.081 & 96000 & 47.671 \\
53000 & 47.994 & 75000 & 48.063 & 97000 & 47.617 \\
54000 & 48.046 & 76000 & 47.941 & 98000 & 47.479 \\
55000 & 48.111 & 77000 & 47.978 & 99000 & 47.418 \\
56000 & 48.336 & 78000 & 47.884 & 100000 & 47.420 \\
57000 & 48.432 & 79000 & 47.933 & 101000 & 47.379
\end{tabular}

The program described above was also used in [9] to find the class numbers of all pure cubic fields of the form $Q(\sqrt[3]{r})$, where $r \equiv 17(\bmod 18), r$ is a prime and $r<$ $2 \times 10^{5}$.

12. Acknowledgements. The authors wish to thank the referee for his careful reading of the original manuscript and his many useful suggestions for improvements. They also wish to thank D. H. and Emma Lehmer for making available to them an English translation of [6].

Department of Computer Science

University of Manitoba

Winnipeg, Manitoba, Canada R3T 2N2

1. PIERRE BARRUCAND, H. C. WILlIAMS \& L. BANIUK, "A computational technique for determining the class number of a pure cubic field," Math. Comp., v. 30, 1976, pp. 312-323.

2. B. D. BEACH, H. C. WILLIAMS \& C. R. ZARNKE, "Some computer results on units in quadratic and cubic fields," Proc. Twenty-Fifth Summer Meeting of the Canadian Math. Congress, Lakehead Univ., Thunder Bay, Ont., 1971, pp. 609-648.

3. B. N. DELONE \& D. K. FADDEEV, The Theory of Irrationalities of the Third Degree, Transl. Math. Monographs, Vol. 10, Amer. Math. Soc., Providence, R. I., 1964.

4. TAIRA HONDA, "Pure cubic fields whose class numbers are multiples of three," $J$. Number Theory, v. 3, 1971, pp. 7-12. 
5. R. STEINER, "On the units in algebraic number fields," Proc. 6th Manitoba Conference on Numerical Math., 1976, pp. 413-435.

6. G. F. VORONOI, On a Generalization of the Algorithm of Continued Fractions, Doctoral Dissertation, Warsaw, 1896. (Russian)

7. HIDEO WADA, "A table of fundamental units of purely cubic fields," Proc. Japan Acad., v. 46, 1970, pp. 1135-1140.

8. H. C. WILLIAMS, "Certain pure cubic fields with class number one," Math. Comp., v. 31, 1977, pp. 578-580.

9. H. C. WILLIAMS \& D. SHANKS, "A note on class-number one in pure cubic fields," Math. Comp., v. 33, 1979, pp. 1317-1320. 